$10: 30-94850$

SANDIA REPORT

SAND94-2361 • UC-706

Unlimited Release

Printed October 1994

\title{
Electromagnetic Scattering from Buried Objects
}

Billy C. Brock, Kurt W. Sorensen

Prepared by Sandla National Laboratories

Albuquerque, New Mexico 87185 and Livermore, California 94550 for the United States Department of Energy under Contract DE-AC04-94AL85000

Approved for public release; distribution is unlimited. 
Issued by Sandia National Laboratories, operated for the United States Department of Energy by Sandia Corporation.

NOTICE: This report was prepared as an account of work sponsored by an agency of the United States Government. Neither the United States Government nor any agency thereof, nor any of their employees, nor any of their contractors, subcontractors, or their employees, makes any warranty, express or implied, or assumes any legal liability or responsibility for the accuracy, completeness, or usefulness of any information, apparatus, product, or process disclosed, or represents that its use would not infringe privately owned rights. Reference herein to any specific commercial product, process, or service by trade name, trademark, manufacturer, or otherwise, does not necessarily constitute or imply its endorsement, recommendation, or favoring by the United States Government, any agency thereof or any of their contractors or subcontractors. The views and opinions expressed herein do not necessarily state or reflect those of the United States Government, any agency thereof or any of their contractors.

Printed in the United States of America. This report has been reproduced directly from the best available copy.

Available to DOE and DOE contractors from Office of Scientific and Technical Information

PO Box 62

Oak Ridge, TN 37831

Prices available from (615) 576-8401, FTS 626-8401

Available to the public from

National Technical Information Service

US Department of Commerce

5285 Port Royal RD

Springfield, VA 22161

NTIS price codes

Printed copy: A04

Microfiche copy: A06 


\section{DISCLAIMER}

Portions of this document may be illegible in electronic image products. Images are produced from the best available original document. 


\title{
Electromagnetic Scattering from Buried Objects
}

\author{
Billy C. Brock \\ Kurt W. Sorensen \\ Sandia National Laboratories \\ Antenna Development Department \\ Albuquerque, New Mexico 87185-0531
}

\begin{abstract}
Radar imaging and detection of objects buried in soil has potentially important applications in the areas of nonproliferation of weapons, environmental monitoring, hazardous-waste site location and assessment, and even archeology. In order to understand and exploit this potential, it is first necessary to understand how the soil responds to an electromagnetic wave, and how targets buried within the soil scatter the electromagnetic wave. We examine the response of the soil to a short pulse, and illustrate the roll of the complex dielectric permittivity of the soil in determining radar range resolution. This leads to a concept of an optimum frequency and bandwidth for imaging in a particular soil. We then propose a new definition for radar cross section which is consistent with the modified radar equation for use with buried targets. This radar cross section plays the same roll in the modified radar equation as the traditional radar cross section does in the free-space radar equation, and is directly comparable to it. The radar cross section of several canonical objects in lossy media is derived, and examples are given for several object/soil combinations.
\end{abstract}

\section{MASTER}


This Page Intentionally Blank 


\section{Table of Contents}

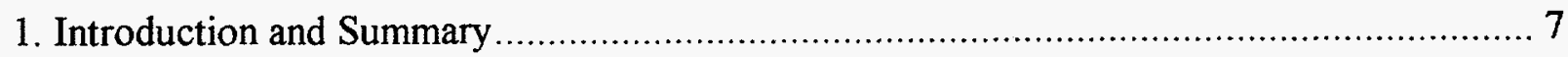

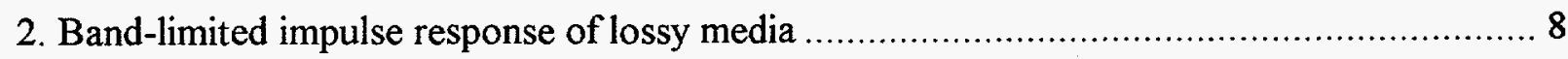

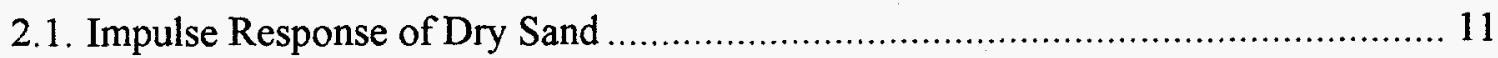

2.2. Impulse Response of San Antonio Clay Loam With 5\% Water ........................... 12

2.3. Effect of Increased Water Content ............................................................... 13

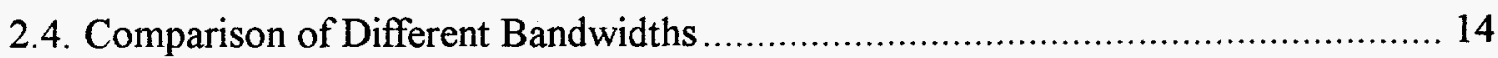

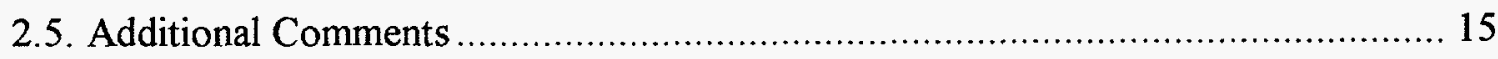

3. Definition of Radar Cross Section in Lossy Media ...................................................... 15

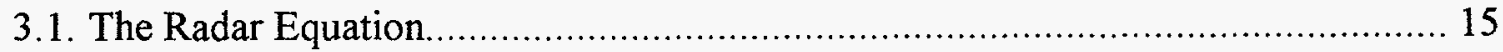

3.2. Modification of the Radar Equation for Lossy Media ....................................... 16

3.3. New Definition of Radar Cross Section in Lossy Media ..................................... 17

4. Radar Cross Section of a Conducting Flat Plate Embedded in Lossy Media ....................... 18

4.1. Calculated Radar Cross Section for Buried Flat Plates ........................................ 24

5. Radar Cross Section of a Perfectly Conducting Circular Cylinder Embedded in

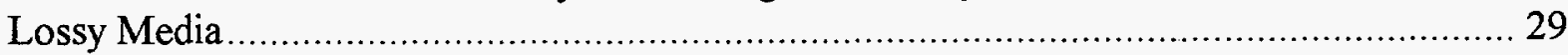

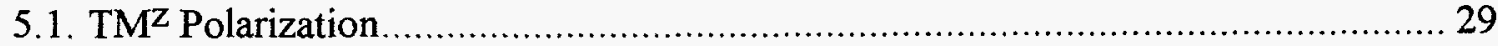

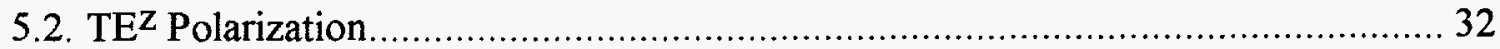

5.3. Calculated Radar Cross Section for Buried Cylinders ........................................... 34

6. Radar Cross Section of a Perfectly Conducting Sphere Embedded in Lossy Media .............. 38

6.1. The Angular Momentum Operator and Vector Spherical Harmonics.................... 40

6.2. Back-scatter Cross Section of Spheres in a Lossy Media.................................... 41

6.3. Behavior of Large Spheres in Lossy Media ....................................................... 41

6.4. Calculated Radar Cross Section for Buried Spheres .......................................... 42

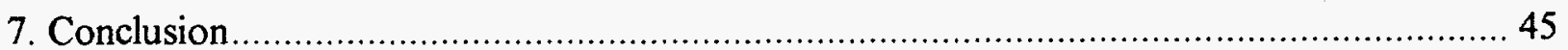

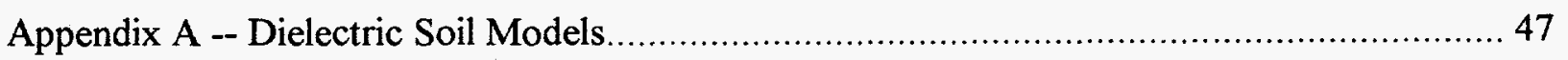

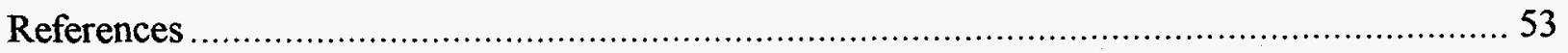


This Page Intentionally Blank 


\section{Introduction and Summary}

The imaging of objects buried in soil has potentially valuable applications in several diverse areas, such as nonproliferation of weapons, environmental monitoring, hazardous-waste site location and assessment, and even archeology. In order to understand and exploit this potential, it is first necessary to understand how the soil responds to an electromagnetic wave, and how targets buried within the soil scatter the electromagnetic wave. A previous study [1] addressed the issue of imaging buried objects in the presence of surface clutter. It showed that an optimum frequency exists, which depends on soil characteristics, burial depth, clutter characteristics, and target type. Although the frequency dependence of the dielectric permittivity of the soil was included at the synthetic-aperture radar (SAR) center frequency, dispersion of the frequency components within the compressed pulse was not included in the analysis.

The two-way impulse response of the soil is important in determining the best operating frequency and bandwidth for a subsurface-imaging SAR. Because of dispersion and loss in the soil, the impulse response is distorted from the free-space impulse response. The following conclusions can be drawn from examination of the soil's impulse response:

- an optimum bandwidth exists;

- loss increases as bandwidth increases;

- very large bandwidths are not useful for imaging objects at large depths;

- in the absence of additional processing, contrast is reduced as depth increases;

- vertical polarization is best for large angles of incidence;

- in the absence of surface clutter, lower frequencies seem best.

In addition to understanding the impulse response of the soil, it is necessary to compute the radar cross section for simple objects buried in lossy media. For convenience, the radar cross section is defined to have a meaning similar to, and consistent with, the free-space radar cross section used with conventional radar analysis. In [1], the radar cross section of buried objects was modeled as the radar cross section in free space, but scaled according to the wavelength in the soil. Here, a more precise model is given. It is shown that, to first order, the scaled model used in [1] is reasonable.

For buried objects, a modified form of the radar equation will be used

$$
P_{r}=P_{t} \frac{G_{t}}{4 \pi R_{t}^{2}} T_{0,1} L\left(R_{t, 2}\right) \frac{\sigma_{\mu \varepsilon}}{4 \pi R_{r}^{2}} L\left(R_{t, 2}\right) T_{1,0} \frac{G_{r} \lambda^{2}}{4 \pi},
$$

where $P_{t}$ is the transmitted power, $G_{t}, G_{r}$ are the gains of the transmitting and receiving antennas respectively, $R_{r}, R_{r}$ are the total ranges (including both the free-space and underground paths) to the target object from the transmitter and receiver respectively, $\sigma_{\mu \varepsilon}$ is the object's radar cross section in the lossy media, $\lambda$ is the signal wavelength, $T_{0,1}$ and $T_{1,0}$ are power-transmission coefficients from one media to the other, and $L\left(R_{t, 2}\right), L\left(R_{r, 2}\right)$ are the power-propagation-loss coefficients along the respective paths, $R_{r, 2}, R_{r, 2}$ from the surface to the object. Here, the radar cross section is designated $\sigma_{\mu \varepsilon}$ to distinguish it from the free-space radar cross section $\sigma$. 
A new definition of radar cross section is required to properly account for the loss in the media, and to allow the losses to be factored as shown in the modified radar equation. The new definition for radar cross section is

$$
\sigma_{\mu \varepsilon}=\lim _{R \rightarrow \infty} 4 \pi R^{2} \frac{\left|e^{\mid \operatorname{Im}(k)(R-a)} \overline{\mathbf{E}}_{s}\right|^{2}}{\left|e^{|\operatorname{lm}(k)| a \mid}\right|^{2}}
$$

where $k$ is the complex wave number, $a$ is the distance from the coordinate origin to the dominant specular point of the target, and $\overline{\mathbf{E}}_{s}$ is the scattered electric field vector. This definition is independent of the object's depth, is consistent with the usual definition for the free-space radar cross section, and can be directly compared with the free-space cross section. Note, however, that while the definition is independent of the object's depth, the scattered field, $\overrightarrow{\mathbf{E}}_{s}$, may not be. We argue that, for many cases of interest, the scattered field can be computed independently of the depth and retain very good accuracy.

The radar cross section for several types of buried objects is derived: flat plates, cylinders, and spheres. These models for radar cross section will find considerable utility in estimating target brightness using the radar equation. However, the complex scattered field is required if the phase history for a SAR image is to be computed. In addition to the radar cross section, expressions for the complex scattered field are also included. Thus, a consistent set of models are derived which can be used with the simple radar equation, or used to generate a complex phase history of buried objects.

Several conclusions can be drawn from the new definition of radar cross section and the models derived for buried objects:

- the appropriate depth for computing losses is the depth to the dominant specular scattering point on the object;

- free-space radar cross sections scaled by the wavelength in the lossy media provide reasonable estimates of the object's buried radar cross section;

- multiple scatterer interactions are less significant for objects buried in lossy media;

- with the new definition, the radar cross section of a buried object behaves much like intuition would predict.

\section{Band-limited impulse response of lossy media}

The band-limited two-way impulse response described here includes both dispersion (phase) and frequency-dependent loss, which are characteristics of soils. The two-way impulse response includes the Fresnel transmission coefficient at the air-soil interface (into and out of the soil) as well as two-way propagation to a given depth in the soil.

The band-limited impulse response is obtained by a Fourier transform of the frequency-domain response of the soil over the appropriate bandwidth, when illuminated by a plane wave. Fig. 1 shows the geometry in which the response is referenced. The half space above the $\hat{\mathbf{x}}-\hat{\mathbf{y}}$ plane is free space $\left(\varepsilon_{0}, \mu_{0}\right)$, and the lossy, dispersive half space below is homogeneous soil $\left(\varepsilon_{s}, \mu_{s}\right)$. 


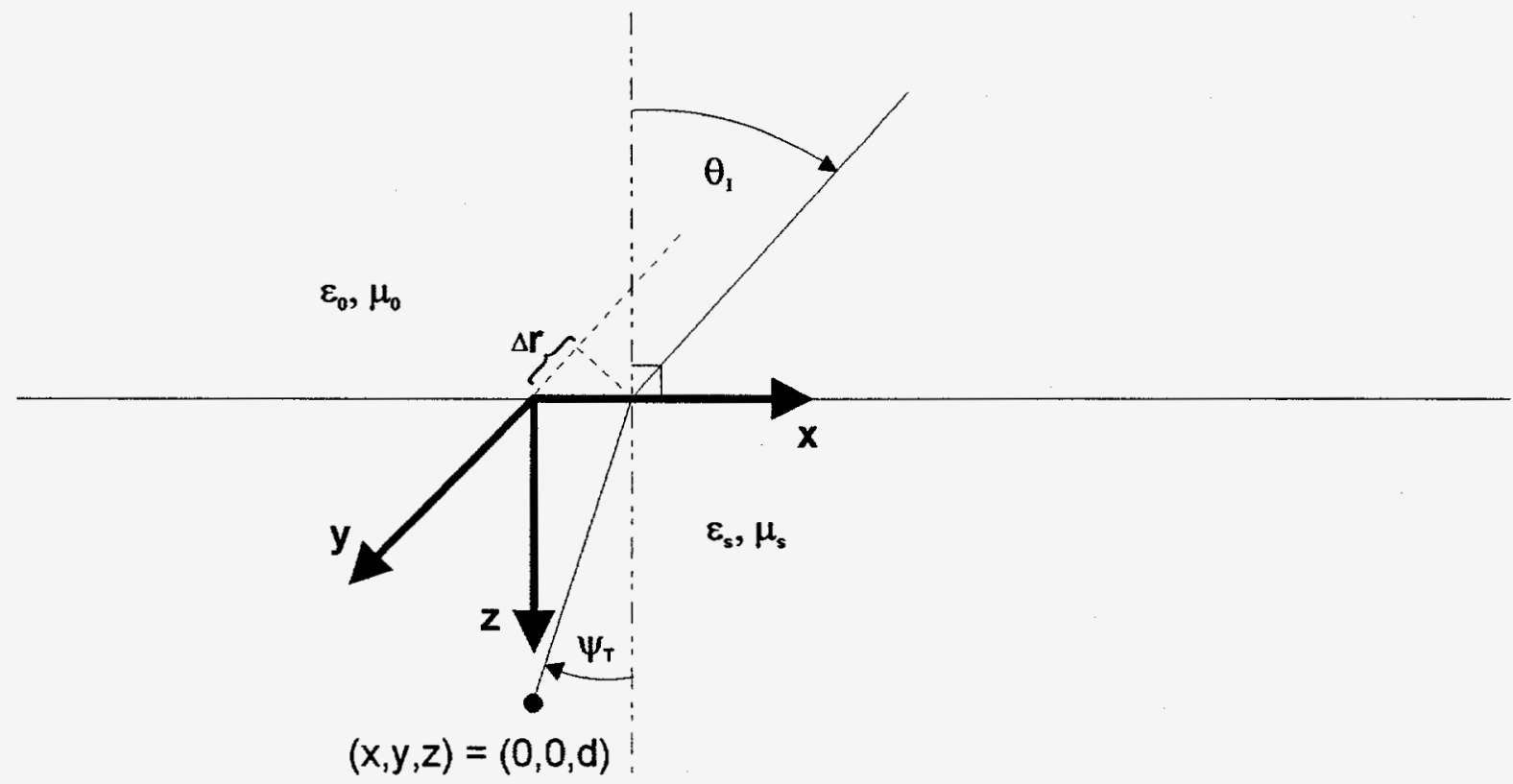

Fig. 1 Geometry illustrating a plane wave incident upon a planar air-soil interface.

Wave numbers are defined in each medium

$$
k_{0}=\frac{\omega}{c}=2 \pi f \sqrt{\varepsilon_{0} \mu_{0}},
$$

and

$$
k_{s}(f)=2 \pi f \sqrt{\varepsilon_{s}(f) \mu_{s}(f)},
$$

where $f$ is the instantaneous frequency. The complex angle of refraction, $\theta_{T}$, is related to the incident angle, $\theta_{I}$, through Snell's law

$$
\sin \theta_{T}=\frac{k_{0}}{k_{s}} \sin \theta_{I} .
$$

The real angle of refraction, $\psi_{T}$, is the angle between the normal to the constant-phase planes and the $\mathrm{z}$-axis [2], and is given by

$$
\psi_{T}=\operatorname{Tan}^{-1}\left[\frac{\operatorname{Re}\left(k_{0}\right) \sin \theta_{T}}{\operatorname{Re}\left(k_{s}\right) \operatorname{Re}\left(\cos \theta_{T}\right)-\operatorname{Im}\left(k_{s}\right) \operatorname{Im}\left(\cos \theta_{T}\right)}\right] .
$$

The free-space path to the point of refraction is shorter than the reference free-space path to the coordinate system origin by an amount

$$
\Delta r=d \tan \psi_{T} \sin \theta_{I}
$$

The two-way band-limited impulse response (referenced to the origin) is 


$$
\begin{aligned}
I(t) & =\frac{1}{\omega_{2}-\omega_{1}} \int_{\omega_{1}}^{\omega_{2}}(1+\Gamma)(1-\Gamma) e^{-j\left(2 k_{s} \frac{d}{\cos \psi_{T}}\left(\sin \psi_{T} \sin \theta_{T}+\cos \psi_{T} \cos \theta_{T}\right)-2 k_{0} \Delta r\right)} e^{j \omega t} d \omega \\
& =\frac{1}{\omega_{2}-\omega_{1}} \int_{\omega_{1}}^{\omega_{2}}(1+\Gamma)(1-\Gamma) e^{-j 2 k_{s} d \cos \theta_{T}} e^{j \omega t} d \omega
\end{aligned}
$$

where $\omega=2 \pi f$. The Fresnel reflection coefficient is

$$
\begin{aligned}
& \Gamma(\omega)=\left\{\begin{array}{ll}
\frac{\eta_{s} \cos \theta_{I}-\eta_{0} \cos \theta_{T}}{\eta_{s} \cos \theta_{I}+\eta_{0} \cos \theta_{T}}, & \text { for perpendicular polarization } \\
\frac{\eta_{s} \cos \theta_{T}-\eta_{0} \cos \theta_{I}}{\eta_{s} \cos \theta_{T}+\eta_{0} \cos \theta_{I}}, & \text { for parallel polarization }
\end{array},\right. \\
& \eta_{0}=\sqrt{\frac{\mu_{0}}{\varepsilon_{0}}},
\end{aligned}
$$

and

$$
\eta_{s}=\sqrt{\frac{\mu_{s}(\omega)}{\varepsilon_{s}(\omega)}} .
$$

The impulse response (6) accounts for both the dispersive and frequency-dependent loss characteristics of the soil, and is normalized so its peak is unity when $k_{s}=k_{0}$.

The quantity plotted in the examples, called the magnitude of the impulse response, is

$$
I_{d B}(t)=20 \log (|I(t)|) \text {. }
$$

Thus, the peak value of $I_{d B}(t)$ corresponds to the two-way loss in $\mathrm{dB}$ to a point scatterer buried at depth $d$ in the soil. Four different soil types will be examined in the examples that follow. The model for the soil dielectric constant is described in Appendix A and in [1]. The soils are listed in Table 1.

\begin{tabular}{|c|c|c|}
\hline \multicolumn{3}{|c|}{$\begin{array}{c}\text { Table 1 } \\
\text { Soil Models }\end{array}$} \\
\hline Soil Model Number & Description & Relative Loss \\
\hline 1 & Dry Sand & Very Low \\
\hline 2 & $\begin{array}{c}\text { San Antonio Clay Loam } \\
5 \% \text { Water by Weight }\end{array}$ & Moderate \\
\hline 3 & $\begin{array}{c}\text { San Antonio Clay Loam } \\
10 \% \text { Water by Weight }\end{array}$ & High \\
\hline 4 & $\begin{array}{c}\text { San Antonio Clay Loam } \\
20 \% \text { Water by Weight }\end{array}$ & Very High \\
\hline
\end{tabular}




\subsection{Impulse Response of Dry Sand}

Fig. 1 illustrates the magnitude of the impulse response $\left(I_{d B}(t)\right)$ for the dry-sand model contained in [1]. Note that the pulse width, as well as the attenuation, increases as the target depth increases. The 3-dB and 10-dB pulse widths are plotted in Fig. 2. The increase in width is not linear with depth for shallow depths, but becomes essentially linear for larger depths.

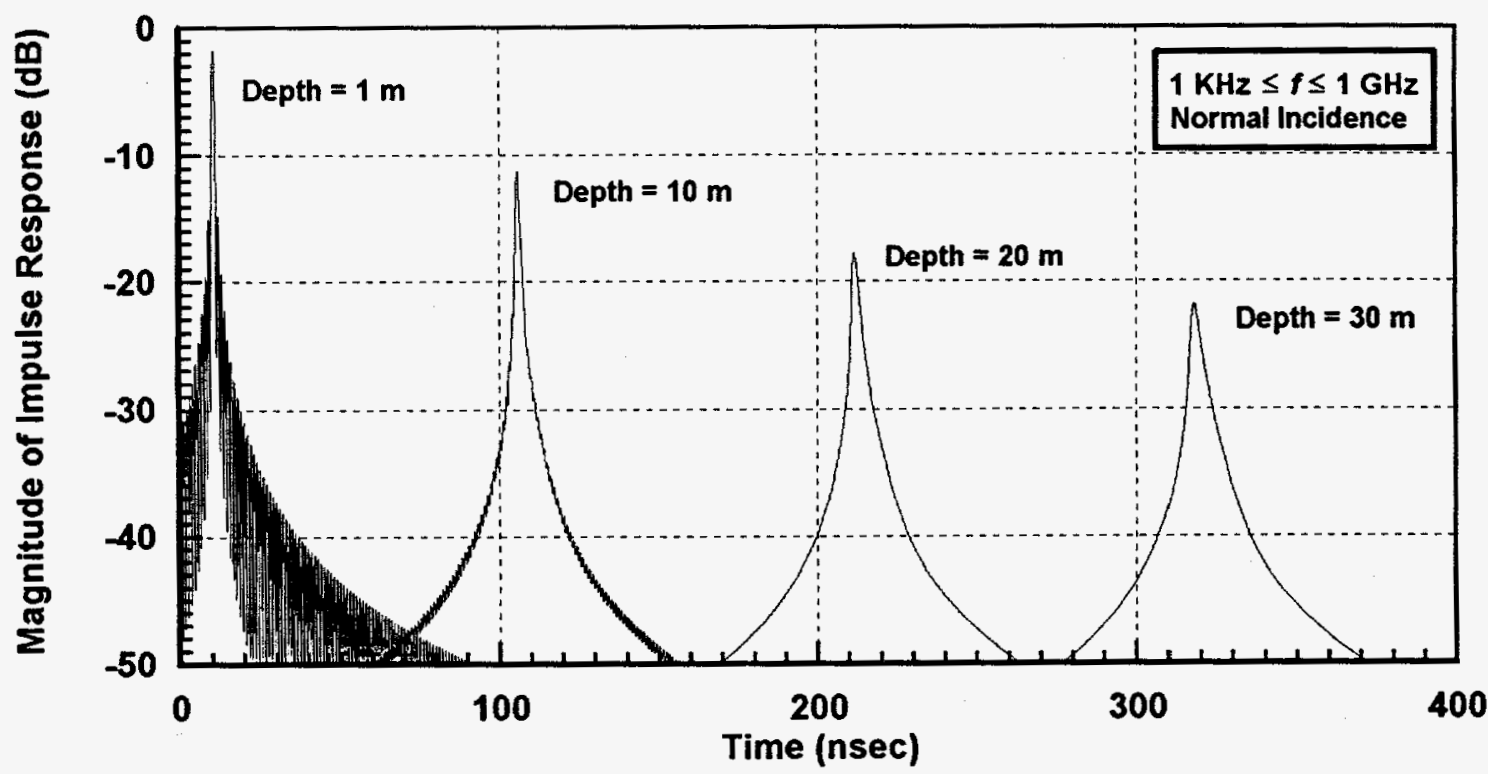

Fig. 1 Magnitude of the band-limited ( $1 \mathrm{KHz} \leq f \leq 1 \mathrm{GHz}$ ) impulse response for dry sand for several depths.

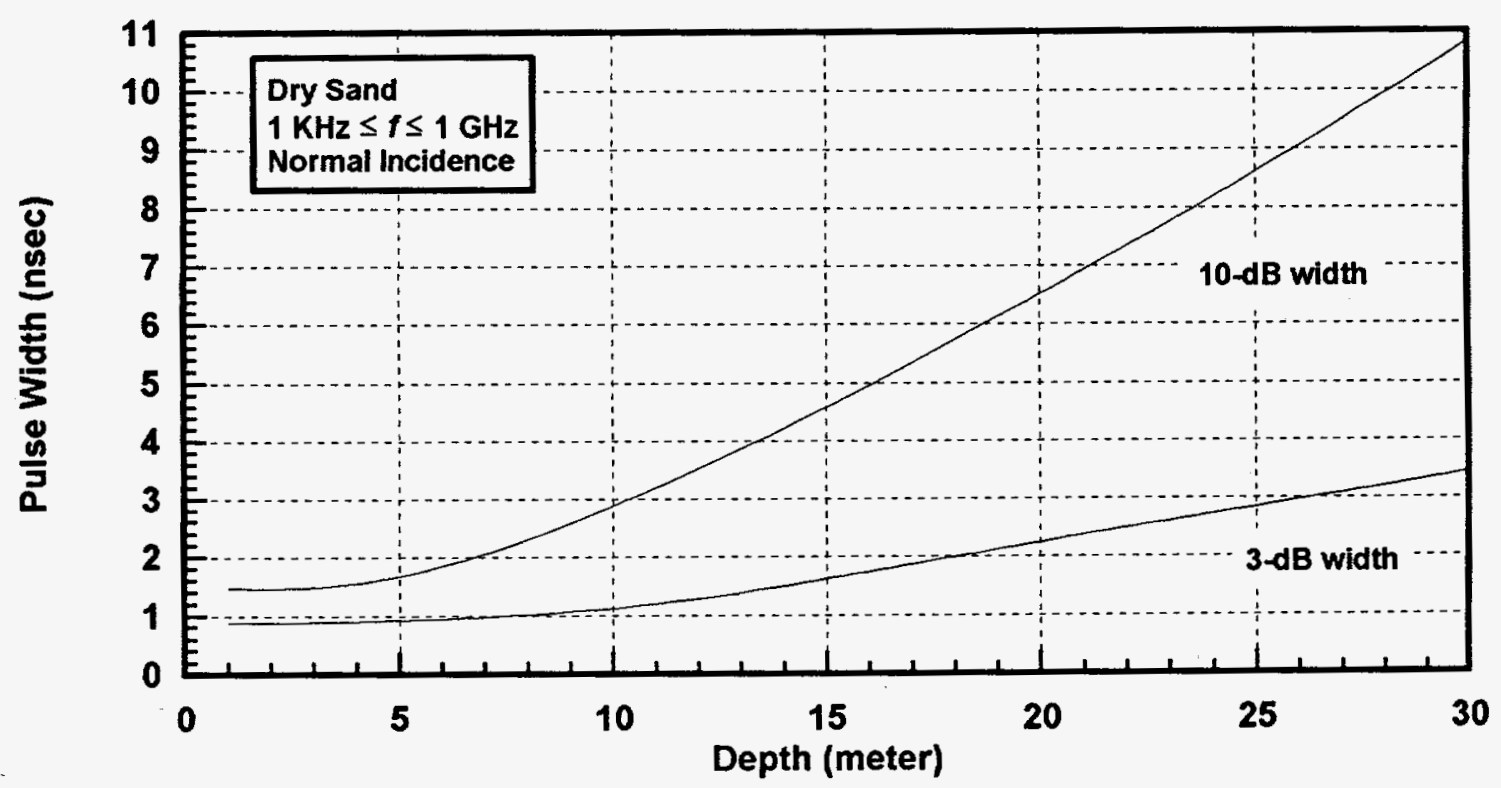

Fig. 2 Width of the band-limited $(1 \mathrm{KHz} \leq f \leq 1 \mathrm{GHz})$ impulse response in dry sand as a function of depth. 


\subsection{Impulse Response of San Antonio Clay Loam With $5 \%$ Water}

The effects of dispersion and loss are much more pronounced in the San Antonio clay loam with $5 \%$ water by weight. The two-way impulse response is illustrated in Fig. 3 for several depths. The 3-dB and 10-dB widths are plotted in Fig. 4. Note that the maximum depth shown is $5 \mathrm{~m}$ rather than $30 \mathrm{~m}$ shown for the sand. Again, the pulse widths show a nonlinear increase as depth increases.

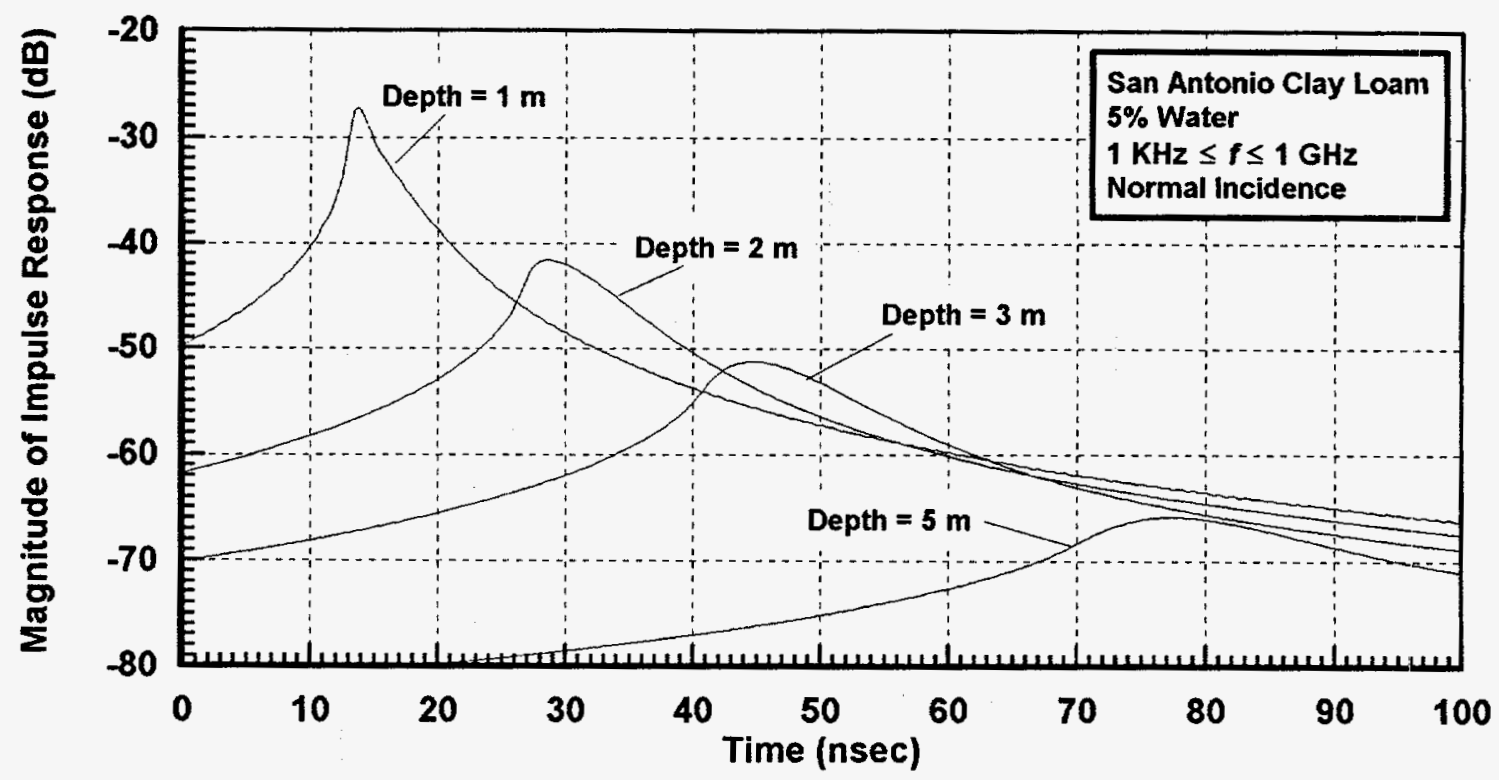

Fig. 3 Two-way impulse response of San Antonio clay loam with 5\% water for various depths.

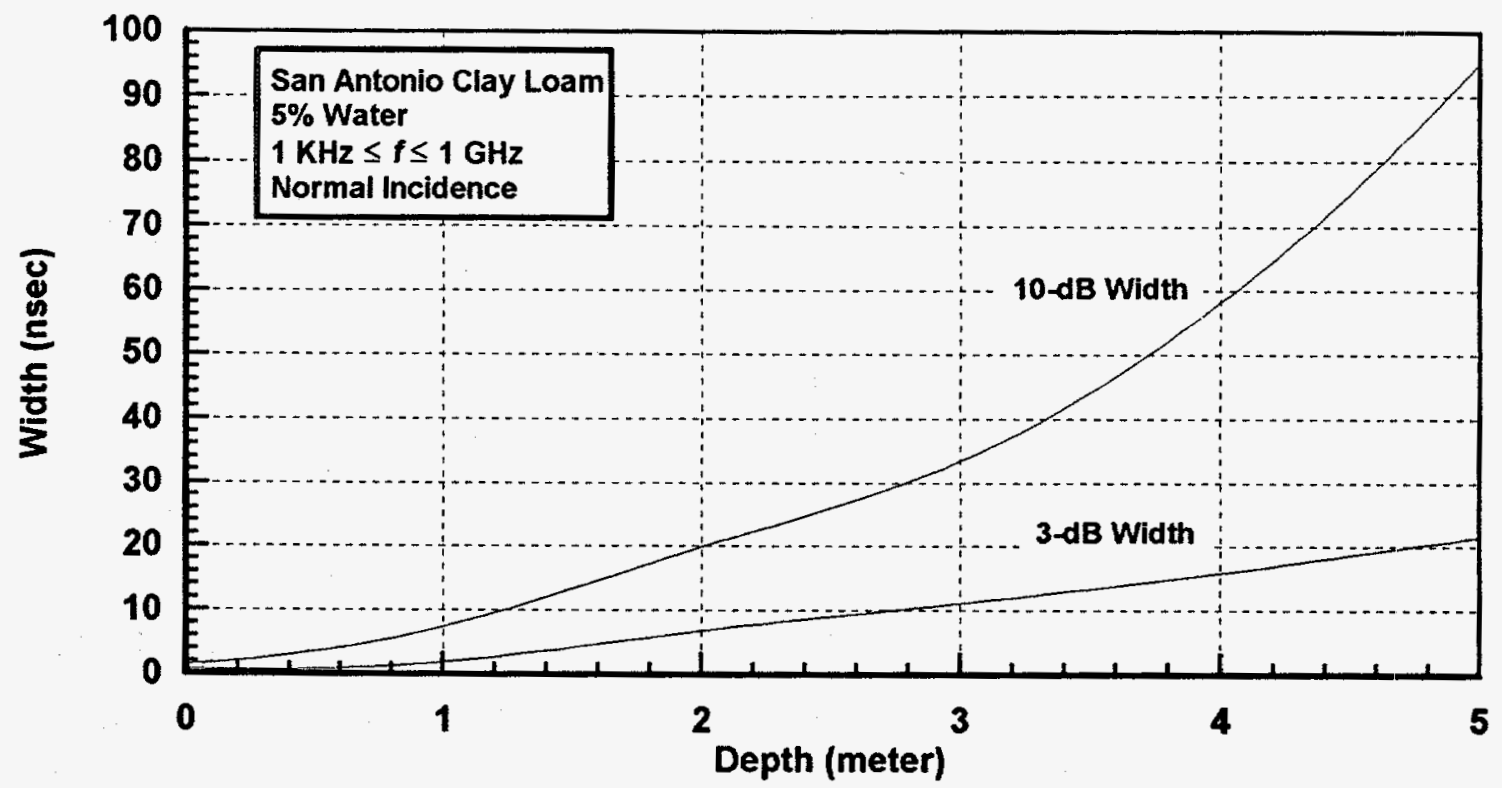

Fig. 4 Width of the impulse response in San Antonio clay loam with $5 \%$ water as a function of depth. 


\subsection{Effect of Increased Water Content}

As the water content is increased, both the losses and dispersion increase. Fig. 5 shows the impulse response for a depth of $1 \mathrm{~m}$ in San Antonio clay loam with $5 \%, 10 \%$, and $20 \%$ water by weight. The increased broadening of the pulse, and the reduced contrast are very apparent as the water content increases. The 3-dB and 10-dB pulse widths are shown in Fig. 6 as a function of water content.

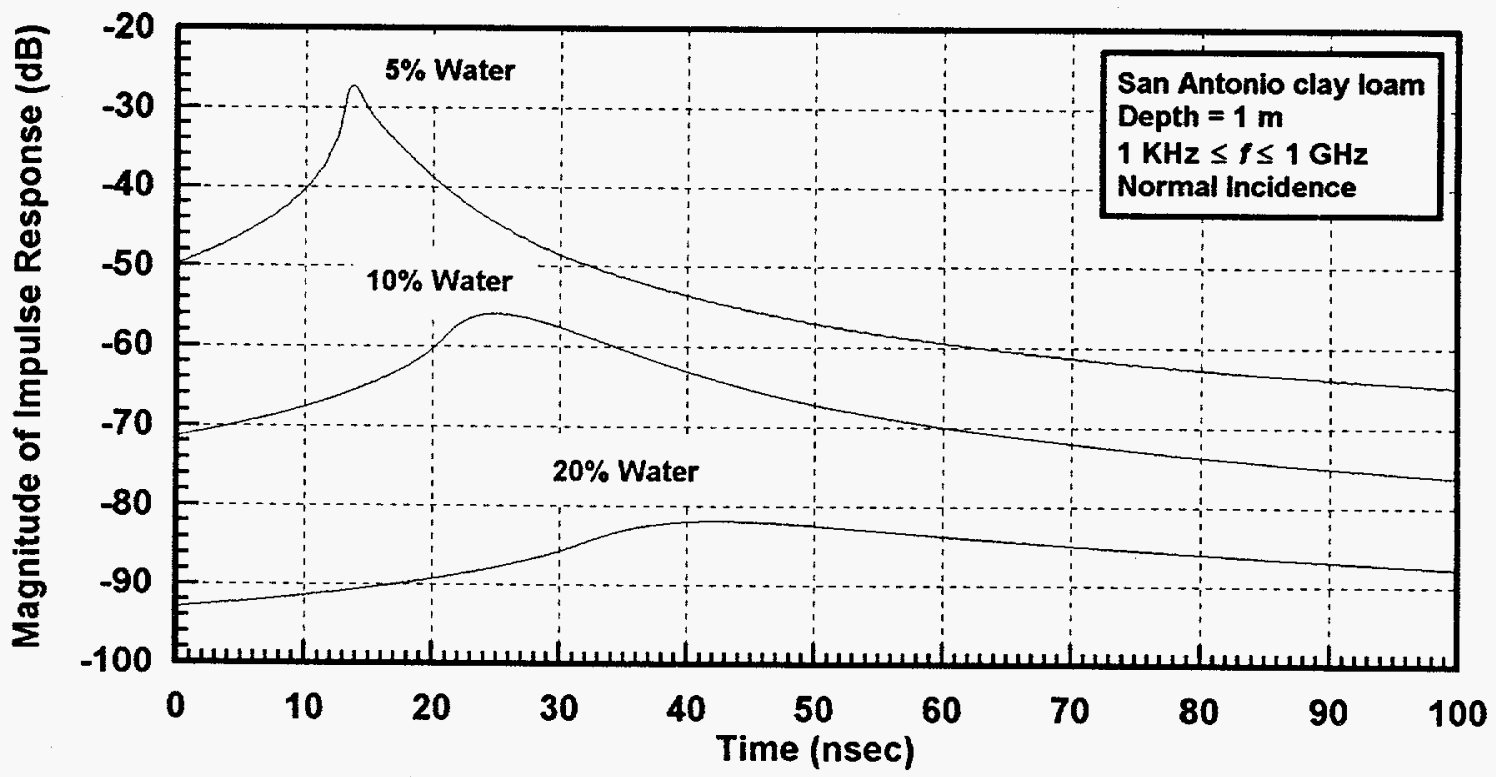

Fig. 5 Impulse response for San Antonio clay loam with 5\%, 10\%, and $20 \%$ water by weight at a depth of 1 meter.

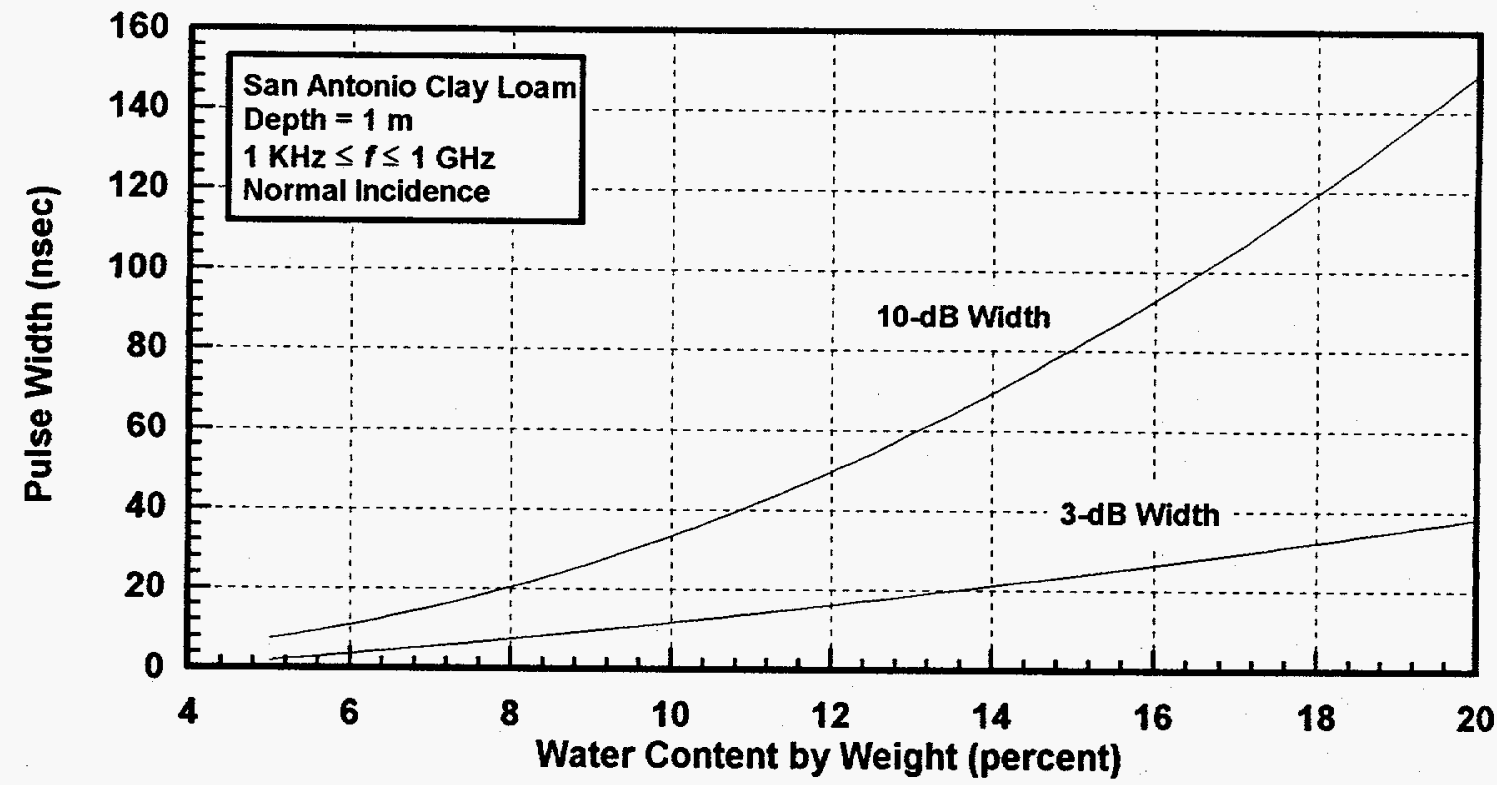

Fig. 6 Impulse response width for San Antonio clay loam at a depth of 1 meter for various water contents. 


\subsection{Comparison of Different Bandwidths}

Because the loss increases significantly with frequency, the soil is effectively a low-pass filter. Very little advantage is gained from very large bandwidths. Fig. 7 presents the band-limited impulse responses for four different bandwidths (from about $50 \mathrm{MHz}$ to $1 \mathrm{GHz}$ ) at a depth of $5 \mathrm{~m}$ in San Antonio clay loam with 5\% water. There is little difference in the pulse shape for $1 \mathrm{GHz}$, $500 \mathrm{MHz}$, and $100 \mathrm{MHz}$ bandwidths. The response with $50 \mathrm{MHz}$ bandwidth is beginning to show significant pulse broadening due to insufficient bandwidth. Thus, it appears that a bandwidth which minimizes loss without sacrificing resolution is probably around 50 to $100 \mathrm{MHz}$ in this soil.

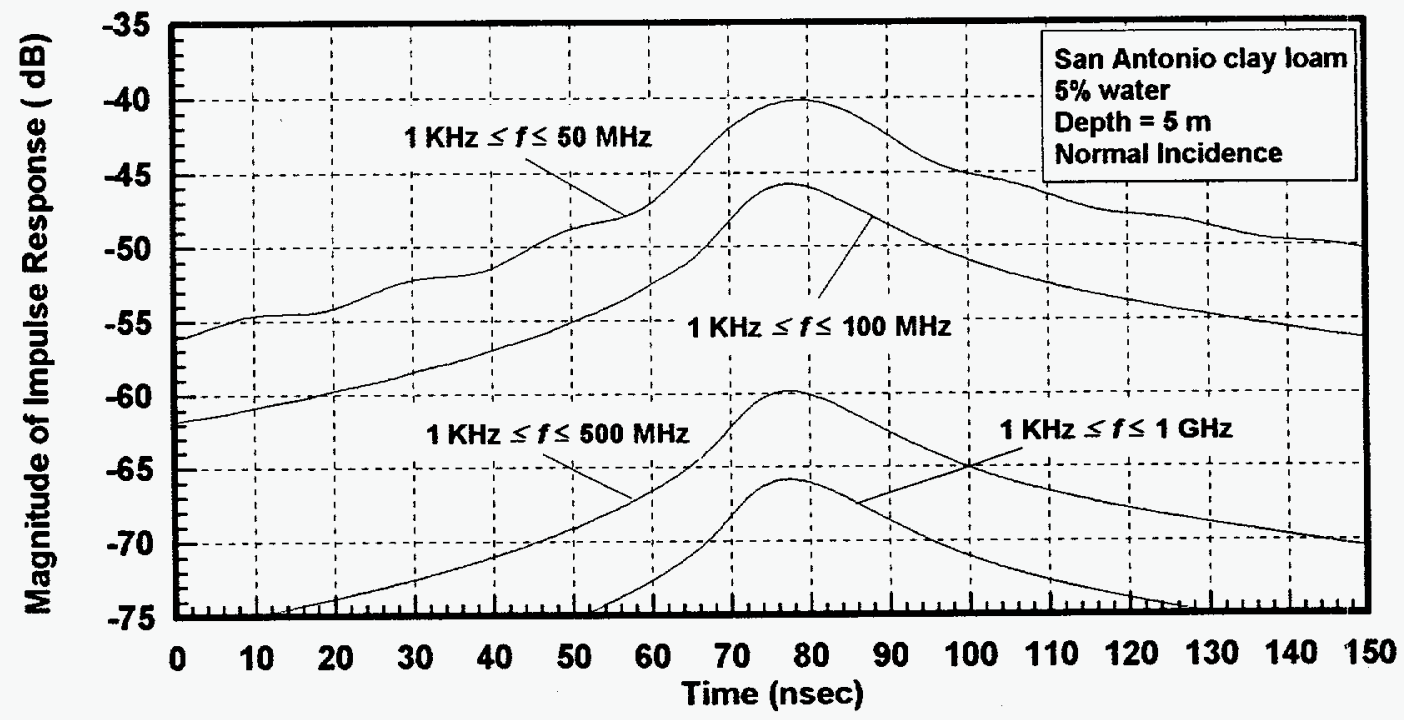

Fig. 7 Band-limited impulse response for several bandwidths at depth of $5 \mathrm{~m}$ in San Antonio clay loam.

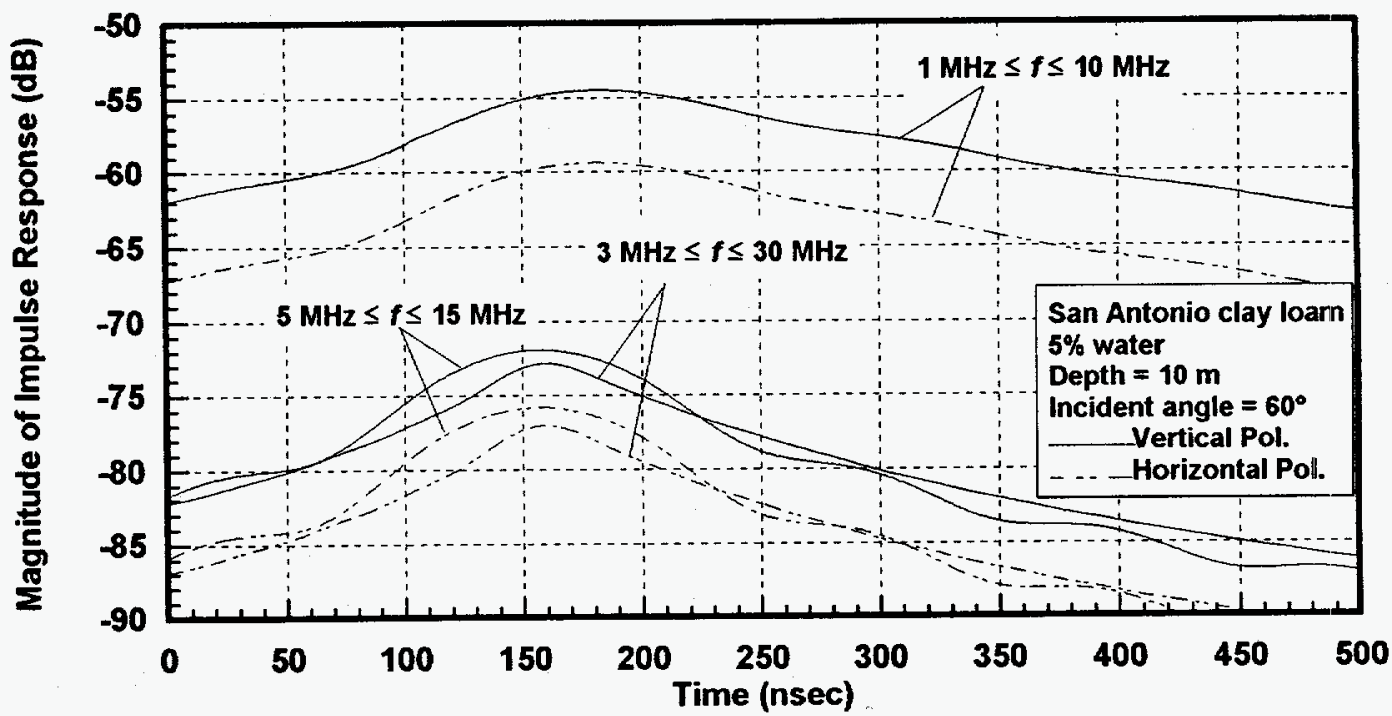

Fig. 8 Band-limited impulse response for three bandwidths at a depth of $10 \mathrm{~m}$ in San Antonio clay loam with 5\% water. 
As the depth is increased, useful bandwidths become smaller, and the useful spectrum is shifted to the lower frequencies. Fig. 8 shows the band-limited impulse response for three different bandwidths at a depth of $10 \mathrm{~m}$ in San Antonio clay loam with $5 \%$ water by weight. Here, the incident angle has been changed from normal incidence $\left(\theta_{I}=0^{\circ}\right)$ to $\theta_{I}=60^{\circ}$, causing a difference between the polarization responses. Vertical polarization shows nearly $5 \mathrm{~dB}$ advantage over horizontal polarization. More than $15 \mathrm{~dB}$ additional loss is suffered with the $5-15 \mathrm{MHz}$ impulse when compared to the $1-10 \mathrm{MHz}$ impulse, even though the bandwidth is almost the same. This is due mainly to the higher center frequency, $10 \mathrm{MHz}$, as compared to $5.5 \mathrm{MHz}$. Increasing both the center frequency and bandwidth to $3-30 \mathrm{MHz}$ improves the resolution with surprisingly little additional loss $(<2 \mathrm{~dB})$ compared to the $5-15 \mathrm{MHz}$ impulse.

\subsection{Additional Comments}

The results reported here illustrate the band-limited impulse response of the soil alone. The effects of surface clutter are not included. As shown in [3], in the presence of surface clutter, the return from a buried object may be difficult to separate from sidelobes of the surface-clutter return. The need for low loss favors low frequencies and small bandwidths, while the need to reduce surface clutter favors large bandwidths and thus higher frequencies [1]. Additional insight and understanding could be obtained by including the clutter return, including loss-compensated window functions (such as described in [3]), and then searching for optimal bandwidths and center frequencies as in [1].

\section{Definition of Radar Cross Section in Lossy Media}

The scattered field used in the definition of the radar cross section, $\sigma_{\mu \varepsilon}$, will be based on uniformplane-wave scattering from an object in an infinite media. In reality, the scattered field from a buried object depends on the interaction with the interface between the lossy media and the air. This interaction can be thought of as multiple reflections between the object and the interface. In a lossy medium, these multiple reflections quickly attenuate to insignificant levels. If they were included in the computation of the radar cross section, it would necessarily be dependent on the depth of burial. However, since it is desirable that the radar cross section, $\sigma_{\mu \varepsilon}$, be independent of the depth of burial, and since the multiple reflections quickly attenuate to insignificant levels in the lossy medium, they will be ignored.

In addition, when a uniform plane wave is incident upon the air-media interface, the transmitted wave becomes nonuniform. However, when the angle of incidence is not too large, the nonuniform plane wave below the surface is nearly uniform. To the extent that this is true, the scattered fields computed here are a good approximation. However, if the computation for the scattered electric field, $\overrightarrow{\mathbf{E}}_{s}$, includes the interaction with the interface and the nonuniformity of the plane wave, then the definition for $\sigma_{\mu \varepsilon}$ will contain no approximations. In this case, the radar cross section will become dependent on both the burial depth and the angle of incidence.

\subsection{The Radar Equation}

In estimating the performance of a conventional radar, the received power $P_{r}$ scattered from an object in free space is computed from the radar equation, 


$$
P_{r}=P_{t} \frac{G_{t}}{4 \pi R_{t}^{2}} \frac{\sigma}{4 \pi R_{r}^{2}} \frac{G_{r} \lambda^{2}}{4 \pi},
$$

where $P_{t}$ is the transmitted power, $G_{t}, G_{r}$ are the gains of the transmitting and receiving antennas respectively, $R_{t}, R_{r}$ are the ranges to the target object from the transmitter and receiver respectively, $\sigma$ is the object's radar cross section, and $\lambda$ is the signal wavelength. The most common application of this equation is for a monostatic radar where $G_{t}=G_{r}=G$ and $R_{r}=R_{r}=R$. The radar cross section, $\sigma$, is defined in the context of the radar equation, and it only really has meaning in this context. It is defined to be the proportionality constant between the incident power density at the object and a total scattered power. This total scattered power is not the true total power scattered from the object, but is the total power necessary to be scattered from an isotropic scatterer which produces the same power density at the receiver as the actual object.

When computing the radar cross section for some object, the approach is to compute the scattered field $\overrightarrow{\mathbf{E}}_{s}$ when given the incident field $\overrightarrow{\mathbf{E}}_{i}$. Common practice is to let the incident field be a plane wave, and reference the cross section to the far field so that

$$
\sigma=\lim _{R \rightarrow \infty} 4 \pi R^{2} \frac{\left|\overline{\mathbf{E}}_{s}\right|^{2}}{\left|\overline{\mathbf{E}}_{i}\right|^{2}} .
$$

\subsection{Modification of the Radar Equation for Lossy Media}

In dealing with buried objects, the definition of radar cross section is more problematic.

Nevertheless, it is still desirable to define a radar equation similar to (11). Referring to Fig. 9, one sees that the computation of the scattered field is complicated by the interface between the two media. Because of the interaction between the object and the interface, the scattered field will contain a dependence on the burial depth, $d$, which is more than simply a propagation loss. However, if it is assumed that the object is buried deep enough in lossy media, the interaction between the object and the interface will be small, and it can be ignored. In this case, it makes sense to define a radar cross section for the object embedded in lossy media of infinite extent.

A radar equation will be defined as follows

$$
P_{r}=P_{t} \frac{G_{t}}{4 \pi R^{2}} T_{0,1} L\left(R_{2}\right) \frac{\sigma_{\mu \varepsilon}}{4 \pi R^{2}} L\left(R_{2}\right) T_{1,0} \frac{G_{r} \lambda^{2}}{4 \pi},
$$

where $R=R_{1}+R_{2}, T_{0,1}$ and $T_{1,0}$ are power-transmission coefficients from one media to the other, and $L\left(R_{2}\right)$ is the power-propagation-loss coefficient along the path from the surface to the object. Here, the radar cross section is designated $\sigma_{\mu \varepsilon}$ to distinguish it from the free-space radar cross section $\sigma$. This radar cross section appears to have basically the same meaning in (13) as the freespace cross section, $\sigma$, in (11). In fact, it has been intentionally defined to be independent of depth so that it can be compared directly with the free-space radar cross section. It will, however, depend on the constitutive parameters, $\mu$ and $\varepsilon$ for the media. It is also understood that $\sigma_{\mu \varepsilon}$ is an approximate radar cross section in that it ignores the effects of the interface between the two 
media. This approximation is good for objects buried in lossy media at a depth where the losses make the interaction terms unimportant.

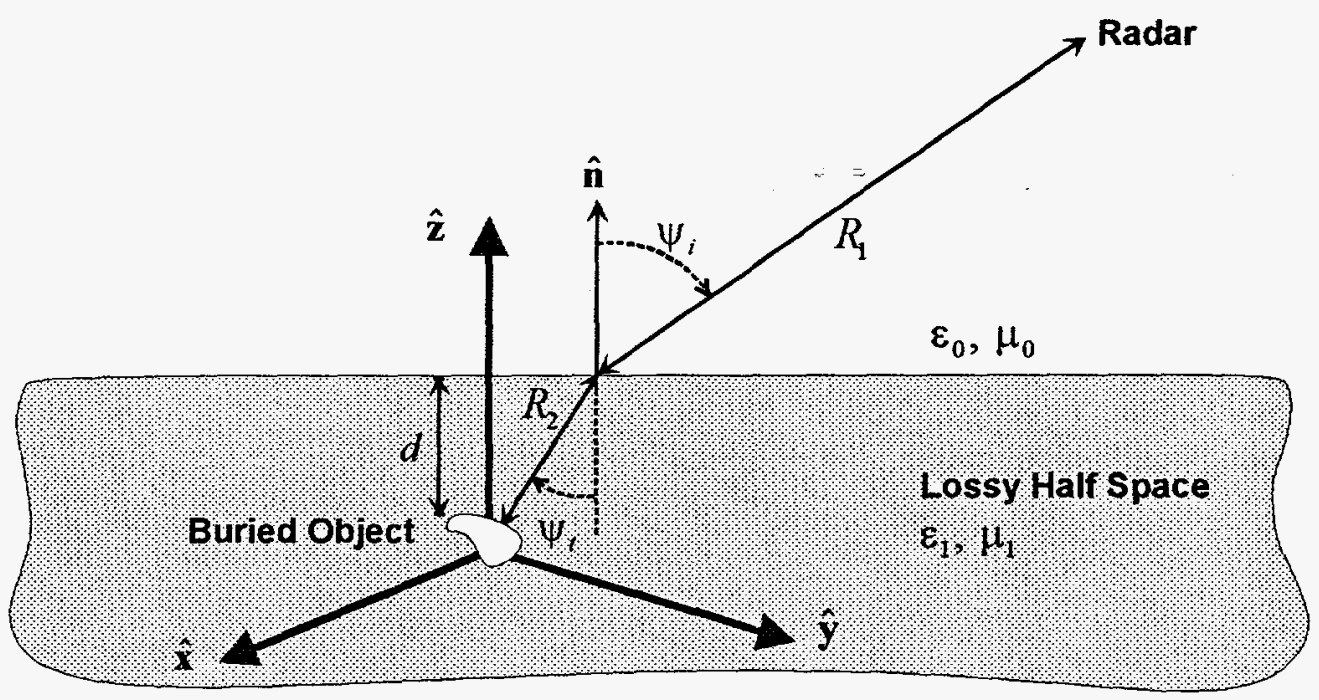

Fig. 9 Arbitrary object buried in a lossy half space is illuminated by airborne radar.

\subsection{New Definition of Radar Cross Section in Lossy Media}

The radar cross section, $\sigma_{\mu \varepsilon}$, will be computed for an object in a lossy full space. However, when the radar cross section is computed from the scattered field, the traditional definition (12) cannot be used. The losses must be carefully factored from the definition of the cross section since they are explicit factors in (13). An additional problem arises with the plane-wave illumination, since a finite amplitude at the origin implies an infinite amplitude at the limit $r \rightarrow \infty$. In fact, if the definition (12) is used, the radar cross section would be zero. Thus, the radar cross section must be normalized in such a way as to remain finite and be consistent with (13). If this is done properly, the new radar cross section will be similar to the free-space radar cross section, and they can be directly compared with each other.

An appropriate solution is to measure the distance $R_{2}$ to the dominant specular point on the object, to reference the incident-illumination amplitude to this point, and to factor from the radar cross section the loss along the path measured from the dominant specular point to the radar. With the new definition for radar cross section, which correctly accounts for the loss, $\sigma_{\mu \varepsilon}$ will be given by

$$
\sigma_{\mu \varepsilon}=\lim _{R \rightarrow \infty} 4 \pi R^{2} \frac{\left.\left|e^{\mid \operatorname{Im}(\overline{\mathbf{k}}(\overline{\mathbf{R}}-\overline{\mathbf{a}}) \mid}\right| \overrightarrow{\mathbf{E}}_{s}\right|^{2}}{\left|\overrightarrow{\mathbf{E}}_{i} e^{|\operatorname{Im}(\overline{\mathbf{k}} \cdot \mathbf{a})|}\right|^{2}},
$$

where $\overrightarrow{\mathbf{a}}$ is the vector from the coordinate-system origin, typically located within the object, to the dominant specular point. The radar cross section, $\sigma_{\mu \varepsilon}$, defined by (14) is consistent with the freespace radar cross section, $\sigma$. Since there is a tendency to compare the cross section of a buried object with its free-space counterpart, this definition is more desirable than a definition which is 
dependent on burial depth. However, it must be emphasized that this definition does not include the interaction between the object and the interface.

Unfortunately, the correct choice for the dominant specular point may not always be obvious. It is possible to choose $\overrightarrow{\mathbf{a}}$ in such a way that the radar cross section as given above will increase (or decrease) exponentially as the object size is increased, counter to intuition derived from experience with free-space radar cross sections. However, if the losses in the modified radar equation are computed to this same point, the computed received power will be correct. The only consequence of a poor choice for the dominant specular point is that the radar cross section no longer behaves intuitively, and it can no longer be compared directly to the free-space radar cross section.

A further complication is associated with the fact that the plane-wave illumination in the lossy half space becomes a nonuniform plane wave for nonnormal incidence. The plane wave is preserved for normal incidence, however, and this case will be treated initially. In addition, the normal to the constant-phase planes will be bent toward the normal to the interface, the amount of bending increasing as the index of refraction, $\eta=\sqrt{\mu_{r} \varepsilon_{r}}$, increases. When the index of refraction is large enough and the incident angle, $\psi$, is small, the constant-phase planes will be nearly parallel to the interface. Thus, since the planes of constant amplitude are almost parallel to the plane of the interface, the uniform-plane-wave approximation will be reasonable when the media has a large index of refraction, and the angle of incidence is not too large.

The approximations can be removed simply by computing the scattered field, $\overrightarrow{\mathbf{E}}_{s}$, with a method that includes the interaction with the interface and the effects of the nonuniform plane wave. In addition to the added complexity of the computation, this approach would have the drawback that the radar cross section will no longer be independent of burial depth or incidence angle. However, the definition (14) is still the appropriate definition for use with the modified radar equation (13), even when the exact scattered field is used.

\section{Radar Cross Section of a Conducting Flat Plate Embedded in Lossy Media}

The first object to be considered is scattering from a three-dimensional, perfectly conducting, rectangular flat plate that is illuminated by a uniform plane wave, and that is embedded in an infinite, lossy medium. Various approaches for calculating the scattering from a flat plate in free space have been successfully applied; the solution derived here parallels the physical optics solution outlined by Balanis [2].

As depicted in Fig. 10, the flat plate has length equal to $a$ and width equal to $b$. As shown in Fig. 11 , the incident plane wave is assumed to have $\mathrm{TE}^{x}$ polarization (electric field transverse to the $\hat{\mathbf{x}}$ axis) and lies on the $\hat{\mathbf{y}}-\hat{\mathbf{z}}$ plane. The incident electric and magnetic fields can therefore be written as

$$
\overrightarrow{\mathbf{E}}^{i}=\eta H_{o}\left(\hat{\mathbf{y}} \cos \theta_{i}+\hat{\mathbf{z}} \sin \theta_{i}\right) e^{-j\left(y \sin \theta_{i}-z \cos \theta_{i}\right)}
$$




$$
\overline{\mathbf{H}}^{i}=\hat{\mathbf{x}} H_{o} e^{-j \gamma\left(y \sin \theta_{i}-z \cos \theta_{j}\right)},
$$

where the propagation constant $\gamma$ is defined as

$$
\gamma=\beta-j \alpha,
$$

in which the real part $\beta$ is interpreted as the phase constant, and the imaginary part $\alpha$ is the attenuation constant.

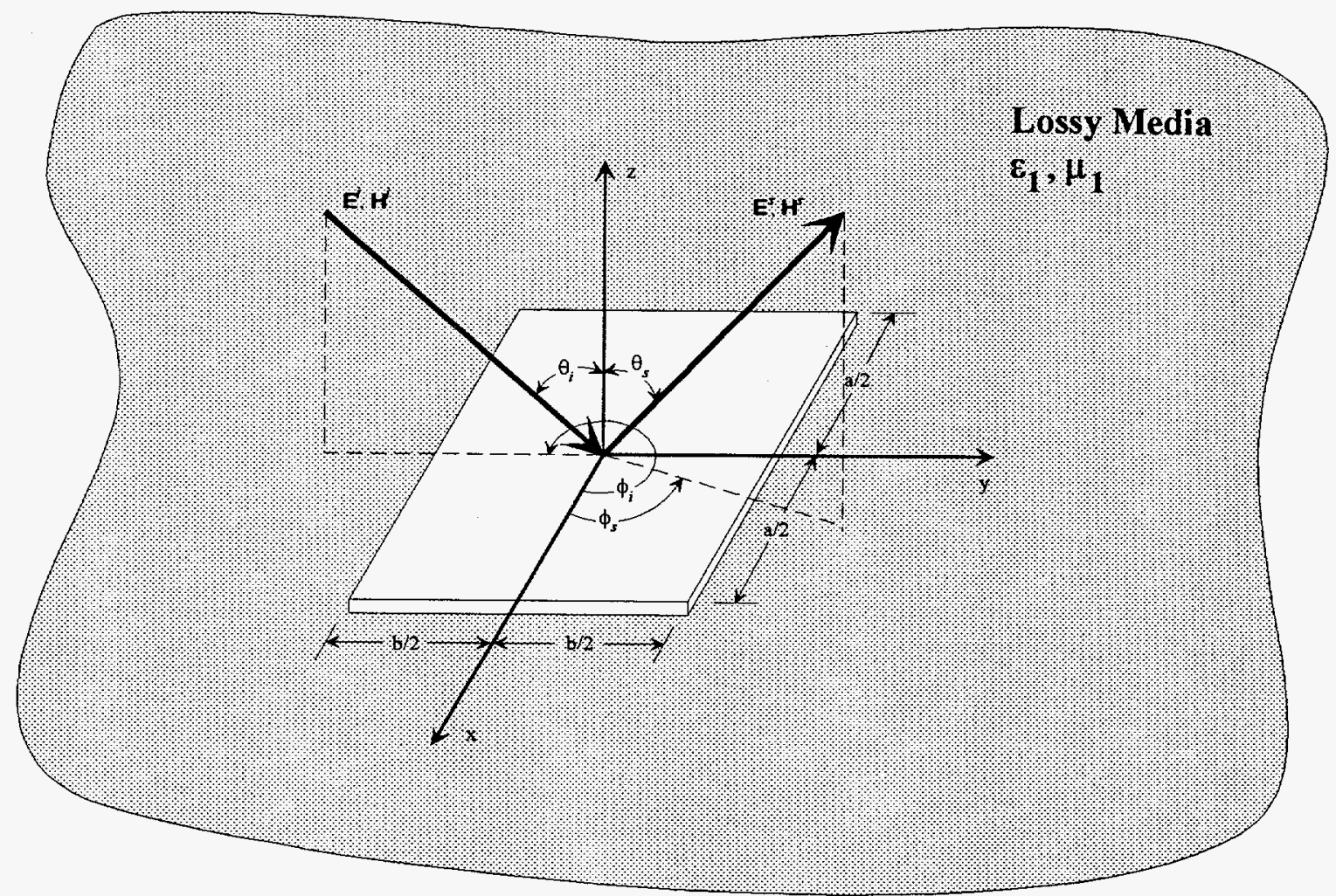

Fig. 10. Uniform plane wave incident on perfectly conducting, rectangular flat plate embedded in lossy media.

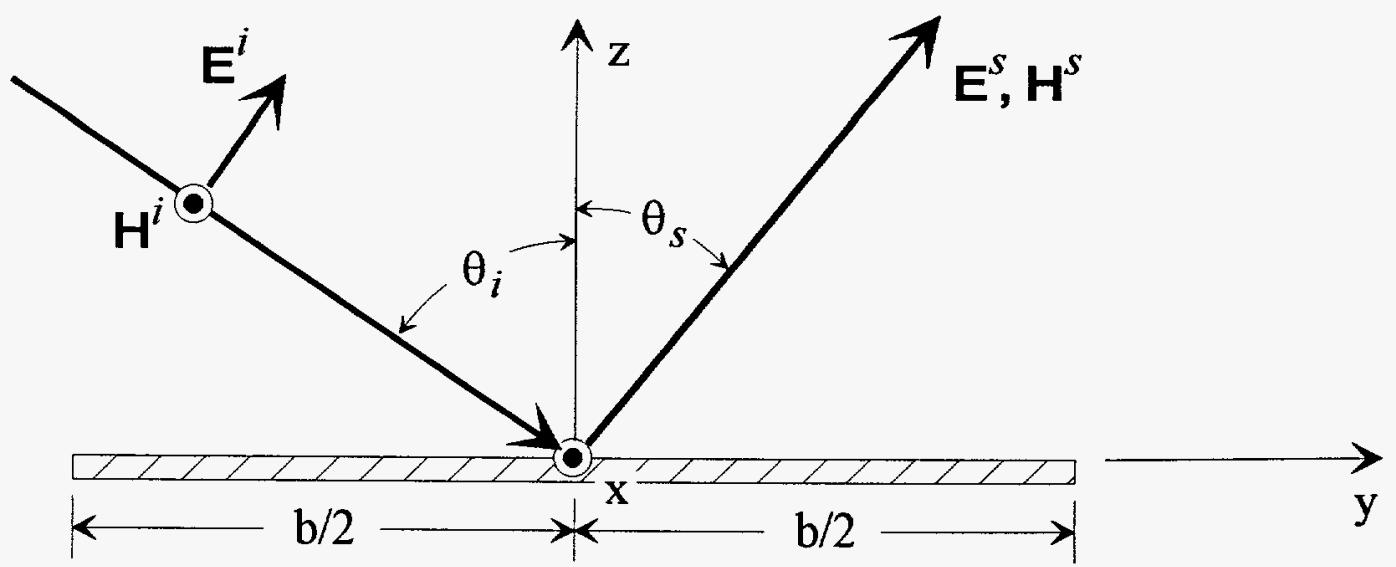

Fig. 11. TEx-polarized uniform plane wave incident on flat plate in $\hat{\mathbf{y}}-\hat{\mathbf{z}}$ plane. 
The total scattered fields are given by

$$
\begin{aligned}
& \overrightarrow{\mathbf{E}}^{s}=-j \omega \overrightarrow{\mathbf{A}}-j \frac{1}{\omega \mu \varepsilon} \nabla(\nabla \cdot \overline{\mathbf{A}})-\frac{1}{\varepsilon} \nabla \times \overrightarrow{\mathbf{F}} \\
& \overline{\mathbf{H}}^{s}=\frac{1}{\mu} \nabla \times \overrightarrow{\mathbf{A}}-j \omega \overrightarrow{\mathbf{F}}-j \frac{1}{\omega \mu \varepsilon} \nabla(\nabla \cdot \overrightarrow{\mathbf{F}}),
\end{aligned}
$$

where $\overline{\mathbf{A}}$ and $\overline{\mathbf{F}}$ are the magnetic and electric vector potentials, respectively, which can be calculated using the expressions

$$
\overline{\mathbf{A}}=\frac{\mu}{4 \pi} \iiint_{V} \vec{J}\left(x^{\prime}, y^{\prime}, z^{\prime}\right) \frac{e^{-j y r}}{r} d v^{\prime}
$$

and

$$
\overrightarrow{\mathbf{F}}=\frac{\varepsilon}{4 \pi} \iiint_{V} \overrightarrow{\mathbf{M}}\left(x^{\prime}, y^{\prime}, z^{\prime}\right) \frac{e^{-j \gamma r}}{r} d v^{\prime},
$$

in which $\overrightarrow{\mathbf{J}}$ and $\overrightarrow{\mathbf{M}}$ are electric and magnetic current densities.

In the far field, the expressions for the electric and magnetic fields simplify considerably. The radial components of the $\overrightarrow{\mathbf{E}}$ and $\overrightarrow{\mathbf{H}}$ fields become negligible, and it has been shown that the farfield components can be written as [2]

$$
\begin{gathered}
E_{r} \cong 0 \\
E_{\theta} \cong-j \omega\left[A_{\theta}+\eta F_{\phi}\right] \\
E_{\phi} \cong-j \omega\left[A_{\phi}-\eta F_{\theta}\right]
\end{gathered}
$$

and

$$
\begin{gathered}
H_{r} \cong 0 \\
H_{\theta} \cong \frac{j \omega}{\eta}\left[A_{\phi}-\eta F_{\theta}\right] \\
H_{\phi} \cong-\frac{j \omega}{\eta}\left[A_{\theta}+\eta F_{\phi}\right],
\end{gathered}
$$

in which the vector potential components have also simplified to

$$
\begin{aligned}
& A_{\theta}=\frac{\mu e^{-j \gamma r}}{4 \pi r} N_{\theta} \\
& A_{\phi}=\frac{\mu e^{-j \gamma r}}{4 \pi r} N_{\phi}
\end{aligned}
$$




$$
\begin{aligned}
& F_{\theta}=\frac{\varepsilon e^{-j \gamma r}}{4 \pi r} L_{\theta} \\
& F_{\phi}=\frac{\varepsilon e^{-j r r}}{4 \pi r} L_{\phi} .
\end{aligned}
$$

The factors $N_{\theta}, N_{\phi}, L_{\theta}$, and $L_{\phi}$ are expressed as

$$
\begin{gathered}
N_{\theta}=\iint_{S}\left(J_{x} \cos \theta \cos \phi+J_{y} \cos \theta \sin \phi-J_{z} \sin \theta\right) e^{j \gamma r} d s^{\prime} \\
N_{\phi}=\iint_{S}\left(-J_{x} \sin \phi+J_{y} \cos \phi\right) e^{j \gamma r^{\prime}} d s^{\prime} \\
L_{\theta}=\iint_{S}\left(M_{x} \cos \theta \cos \phi+M_{y} \cos \theta \sin \phi-M_{z} \sin \theta\right) e^{j \gamma r^{\prime}} d s^{\prime} \\
L_{\phi}=\iint_{S}\left(-M_{x} \sin \phi+M_{y} \cos \phi\right) e^{j \gamma r^{\prime}} d s^{\prime} .
\end{gathered}
$$

For this particular problem, the magnetic current density $(\overline{\mathbf{M}})$ is zero, such that $L_{\theta}=L_{\phi}=0$. Using the physical optics approximation, the current density $(\overline{\mathbf{J}})$ induced on the surface of the plate by the incident plane wave can be represented as

$$
\overrightarrow{\mathbf{J}}_{s}=2 \hat{\mathbf{z}} \times\left.\overrightarrow{\mathbf{H}}^{i}\right|_{\substack{z=0 \\ y=y^{\prime}}}=\hat{\mathbf{y}} 2 H_{o} e^{-i \gamma y^{\prime} \sin \theta_{i}}
$$

The expression given in (36) is exact for a plate of infinite extent, but neglects edge effects for finite plates. The radar cross section predicted by this technique therefore becomes less valid toward grazing incidence of plane waves. A further drawback in using the physical optics approximation to calculate the monostatic radar cross section for a flat plate is that the values calculated are polarization independent; that is, the calculated RCS for both $\mathrm{TE}^{x}$ and $\mathrm{TM}^{x}$ polarizations are equivalent, despite the fact that measurements have shown this to be incorrect, especially near grazing incidence. Nevertheless, physical optics techniques provide results that are quite accurate at and near specular directions [2], and provide a straightforward means of gaining a greater understanding of the scattering behavior of objects (i.e., flat plates) embedded in lossy media.

Combining (22) - (36) allows the scattered field components to now be expressed as follows:

$$
\begin{gathered}
E_{r} \cong 0 \\
E_{\theta} \cong-\frac{j \gamma e^{-j \gamma r}}{4 \pi r} \eta N_{\theta} \\
E_{\phi} \cong-\frac{j \gamma e^{-j \gamma r}}{4 \pi r} \eta N_{\phi} \\
H_{r} \cong 0
\end{gathered}
$$




$$
\begin{gathered}
H_{\theta} \cong \frac{j \gamma e^{-j \gamma r}}{4 \pi r} N_{\phi} \\
H_{\phi} \cong-\frac{j \gamma e^{-j \gamma r}}{4 \pi r} N_{\theta} .
\end{gathered}
$$

Using (32) and the result from (36) that $J_{x}=J_{y}=0, N_{\theta}$ can be expressed as

$$
\begin{aligned}
N_{\theta} & =\iint_{S}\left(2 H_{o} e^{-j y y \sin \theta_{i}}\right) \cos \theta_{s} \sin \phi_{s} e^{j y\left(x^{\prime} \sin \theta \cos \phi+y^{\prime} \sin \theta \sin \phi\right)} d x^{\prime} d y^{\prime} \\
& =2 H_{o} \cos \theta_{s} \sin \phi_{s} \int_{-b / 2}^{b / 2} e^{\left.j y y^{\prime}-\sin \theta_{i}+\sin \theta_{s} \sin \phi_{s}\right)} d y^{\prime} \int_{-a / 2}^{a / 2} e^{j y x^{\prime} \sin \theta_{s} \cos \phi_{s}} d x^{\prime}
\end{aligned}
$$

The integrals included in this expression are of the form

$$
\int_{-c / 2}^{c / 2} e^{j y z} d z=\int_{-c / 2}^{c / 2} e^{j \beta z} e^{\alpha z} d z=\int_{-c / 2}^{c / 2} e^{\alpha z}(\cos \beta z+j \sin \beta z) d z
$$

From [8], it is known that

$$
\begin{aligned}
& \int e^{\alpha z} \sin \beta z d z=\frac{e^{\alpha z}}{\alpha^{2}+\beta^{2}}(\alpha \sin \beta z-\beta \cos \beta z), \\
& \int e^{\alpha z} \cos \beta z d z=\frac{e^{\alpha z}}{\alpha^{2}+\beta^{2}}(\alpha \cos \beta z-\beta \sin \beta z) .
\end{aligned}
$$

Using these relationships, it can be shown that

$$
\int_{-c / 2}^{c / 2} e^{j \gamma z} d z=\frac{j}{\beta-j \alpha}\left[e^{-\frac{c}{2}(\alpha+j \beta)}-e^{\frac{c}{2}(\alpha+j \beta)}\right] .
$$

The use of (47) allows the integrals in the expression for $N_{\theta}$ to now be easily evaluated. That is, setting

$$
Y=\int_{-b / 2}^{b / 2} e^{j y\left(-\sin \theta_{i}+\sin \theta_{s} \sin \phi_{s}\right)} d y^{\prime}
$$

and letting

$$
\begin{gathered}
\beta^{\prime}=\beta\left(\sin \theta_{s} \sin \phi_{s}-\sin \theta_{i}\right) \\
\alpha^{\prime}=\alpha\left(\sin \theta_{s} \sin \phi_{s}-\sin \theta_{i}\right),
\end{gathered}
$$

the results of (47) allow the integral in (48) to be evaluated by observation, giving the result that

$$
Y=\frac{j}{\beta^{\prime}-j \alpha^{\prime}}\left[e^{-\frac{b}{2}\left(\alpha^{\prime}+j \beta^{\prime}\right)}-e^{\frac{b}{2}\left(\alpha^{\prime}+j \beta\right)}\right] .
$$

The second integral in the expression for $N_{\ominus}$ can be similarly evaluated by setting

$$
X=\int_{-a / 2}^{a / 2} e^{j x^{\prime} \sin \theta_{s} \cos \phi_{s}} d x^{\prime}
$$

and letting 


$$
\begin{gathered}
\beta^{\prime \prime}=\beta \sin \theta_{s} \cos \phi_{s} \\
\alpha^{\prime \prime}=\alpha \sin \theta_{s} \cos \phi_{s},
\end{gathered}
$$

such that

$$
X=\frac{j}{\beta^{\prime \prime}-j \alpha^{\prime \prime}}\left[e^{-\frac{a}{2}\left(\alpha^{*}+j \beta^{*}\right)}-e^{\frac{a}{2}\left(\alpha^{*}+j \beta^{*}\right)}\right] .
$$

The final expression for $N_{\ominus}$ can therefore be written as

$$
N_{\theta}=2 H_{o} X Y \cos \theta_{s} \sin \phi_{s} .
$$

Using these results, the expression for $N_{\phi}$ can also now similarly be written as

$$
N_{\phi}=2 H_{o} X Y \cos \phi_{s} \text {. }
$$

Having solved for $N_{\theta}$ and $N_{\phi}$, the scattered fields are then obtained using Equations (37) - (42), such that

$$
\begin{gathered}
E_{r} \cong 0 \\
E_{\theta}^{s} \cong-j \eta \frac{\gamma H_{o}}{2 \pi r}\left(X Y \cos \theta_{s} \sin \phi_{s}\right) e^{-j \gamma r} \\
E_{\phi}^{s} \cong-j \eta \frac{\gamma H_{o}}{2 \pi r}\left(X Y \cos \phi_{s}\right) e^{-j \gamma r} \\
H_{r} \cong 0 \\
H_{\theta}^{s} \cong-\frac{E_{\phi}^{s}}{\eta} \\
H_{\phi}^{s} \cong \frac{E_{\theta}^{s}}{\eta}
\end{gathered}
$$

As seen in Fig. 10, the plane of the incident electromagnetic field for this problem is defined as

$$
\phi_{i}=\frac{3 \pi}{2}, \quad 0 \leq \theta_{i} \leq \frac{\pi}{2} .
$$

For the purposes of this study, the interest is in monostatic backscattering from the plate, meaning that

$$
\phi_{s}=\phi_{i}=\frac{3 \pi}{2}, \theta_{s}=\theta_{i} .
$$

From (60) it can be seen that in the backscattering direction $\left(\phi_{s}=\frac{3 \pi}{2}\right), E_{\phi}^{s} \cong 0$; hence, the total backscattered field $\left(\overrightarrow{\mathbf{E}}^{s}\right)$ is equivalent to $E_{\theta}^{s}$. It must also be noted that the factor $X$ in Equation 
(59) requires further investigation, since at $\phi_{s}=\frac{3 \pi}{2}, X \rightarrow 0 / 0$. Letting $f\left(\phi_{s}\right)=$ numerator of $X$, $g\left(\phi_{s}\right)=$ denominator of $X$, substituting in for $\alpha^{\prime \prime}$ and $\beta^{\prime \prime}$, and invoking L'Hospital's rule, it can be found that

$$
\frac{f^{\prime}\left(\phi_{s}=\frac{3 \pi}{2}\right)}{g^{\prime}\left(\phi_{s}=\frac{3 \pi}{2}\right)}=\frac{a(\beta-j \alpha) \sin \theta_{s}}{(\beta-j \alpha) \sin \theta_{s}}=a,
$$

such that $X_{\theta_{s}=\frac{3 \pi}{2}} \rightarrow a$. Similarly, it may be seen from (51) that L'Hospital's rule must also be invoked to ascertain the value of the factor $Y$ at normal plane wave incidence $\left(\theta_{i}=0^{\circ}\right)$. By doing so, it can be found that $Y_{\theta_{s}=0^{\circ}} \rightarrow b$. The backscattered electric field can now be expressed as

$$
E_{\theta}^{s} \cong j \eta \frac{\gamma H_{o}}{2 \pi r}\left(a Y \cos \theta_{s}\right) e^{-j \gamma r},
$$

where $Y$ is as given in Equation (51), with (since $\theta_{s}=\theta_{i}$ )

$$
\begin{gathered}
\beta^{\prime}=-2 \beta \sin \theta_{s} \\
\alpha^{\prime}=-2 \alpha \sin \theta_{s} .
\end{gathered}
$$

Utilizing the new definition of radar cross section as given in (14), in which the amplitude of the incident plane wave illumination is referenced to the dominant scattering point, the radar cross section of the flat plate can thus be expressed as

$$
\sigma_{\mu \varepsilon}=\left\{\begin{array}{l}
\frac{a^{2}}{\pi} \mid \gamma Y e^{-\left.2 d|\operatorname{mm}(\gamma)|\right|^{2}} \cos ^{2} \theta_{s} \text { for } \theta_{s} \neq 0 \\
\frac{a^{2} b^{2}}{\pi}|\gamma|^{2} \text { for } \theta_{s}=0
\end{array},\right.
$$

The factor $d$ in (67) is defined as the distance from the coordinate system origin (center of the flat plate) to the dominant scattering point of the target. When the plate is oriented normally to the direction of the incident wave $\left(\theta_{s}=\theta_{i}=0\right)$, a specular reflection occurs, and $d=0$. However, as the incidence angle varies from $\theta_{s}=0$, the dominant source of energy scattered back to the radar is that diffracted from the edges of the flat plate, such that

$$
d=\frac{b}{2} \sin \theta_{s} .
$$

\subsection{Calculated Radar Cross Section for Buried Flat Plates}

Fig. 12 - 15 show the calculated radar cross section for a $5 \lambda \times 5 \lambda(\lambda=$ free space wavelength) rectangular flat plate embedded in four different types of lossy soil (as defined in Table 1) as a function of aspect angle $(-\pi / 2 \leq \theta \leq \pi / 2)$ at a frequency of $1.0 \mathrm{GHz}$. Fig. 16 compares the 
calculated radar cross section for the same $5 \lambda \times 5 \lambda$ flat plate located in a lossless medium (free space) with the calculated radar cross section of the plate embedded in soil 4.

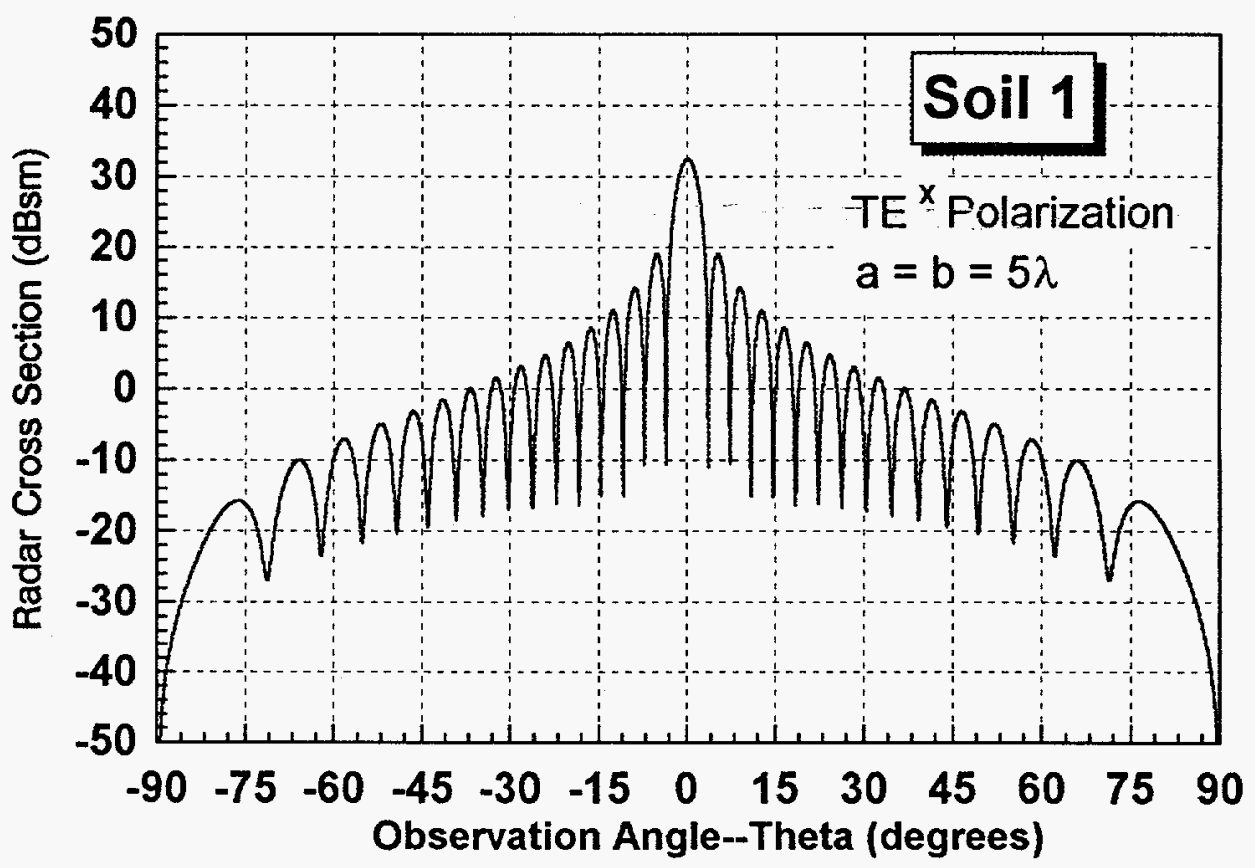

Fig. 3. Principle plane monostatic radar cross section for a $5 \lambda \times 5 \lambda$ rectangular flat plate embedded in dry sand.

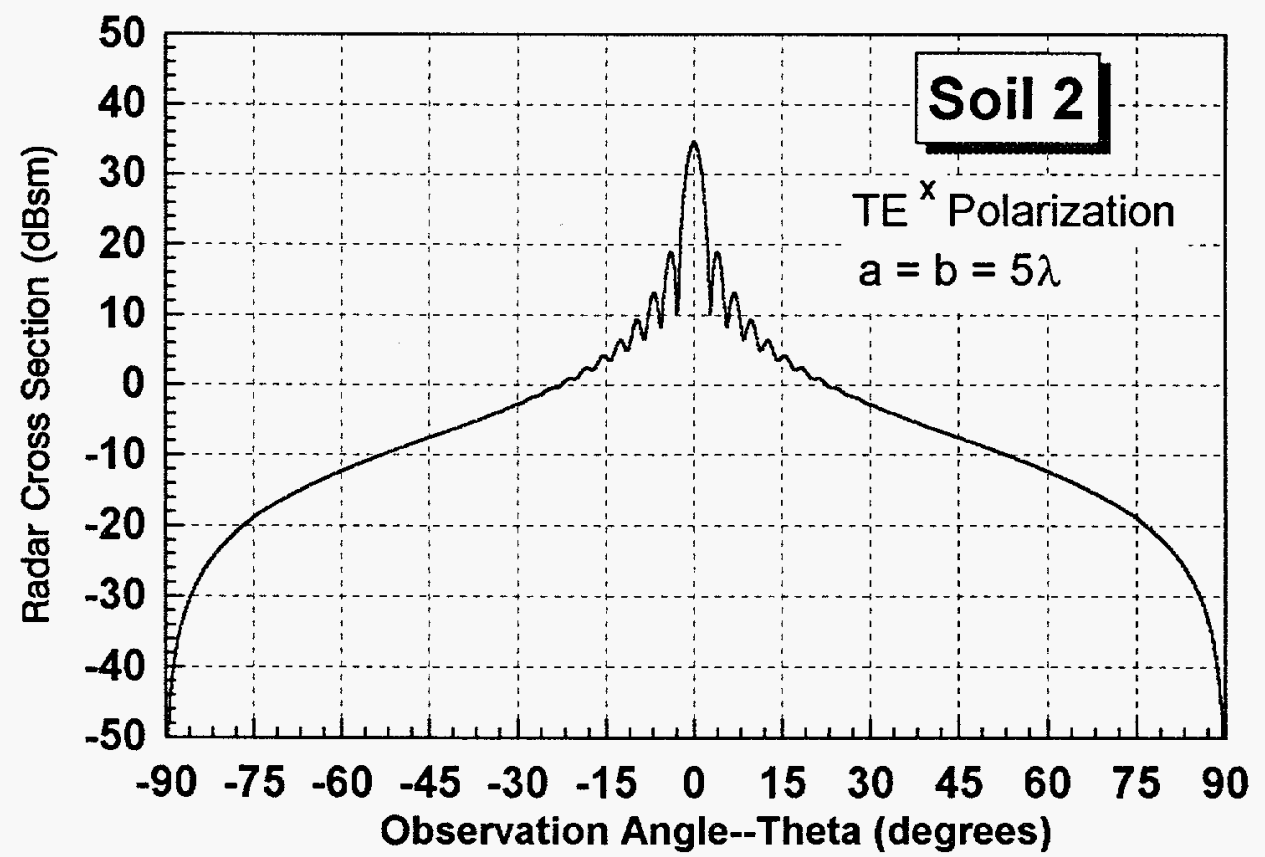

Fig. 4. Principle plane monostatic radar cross section for a $5 \lambda \times 5 \lambda$ rectangular flat plate embedded in San Antonio clay loam with $5 \%$ water. 


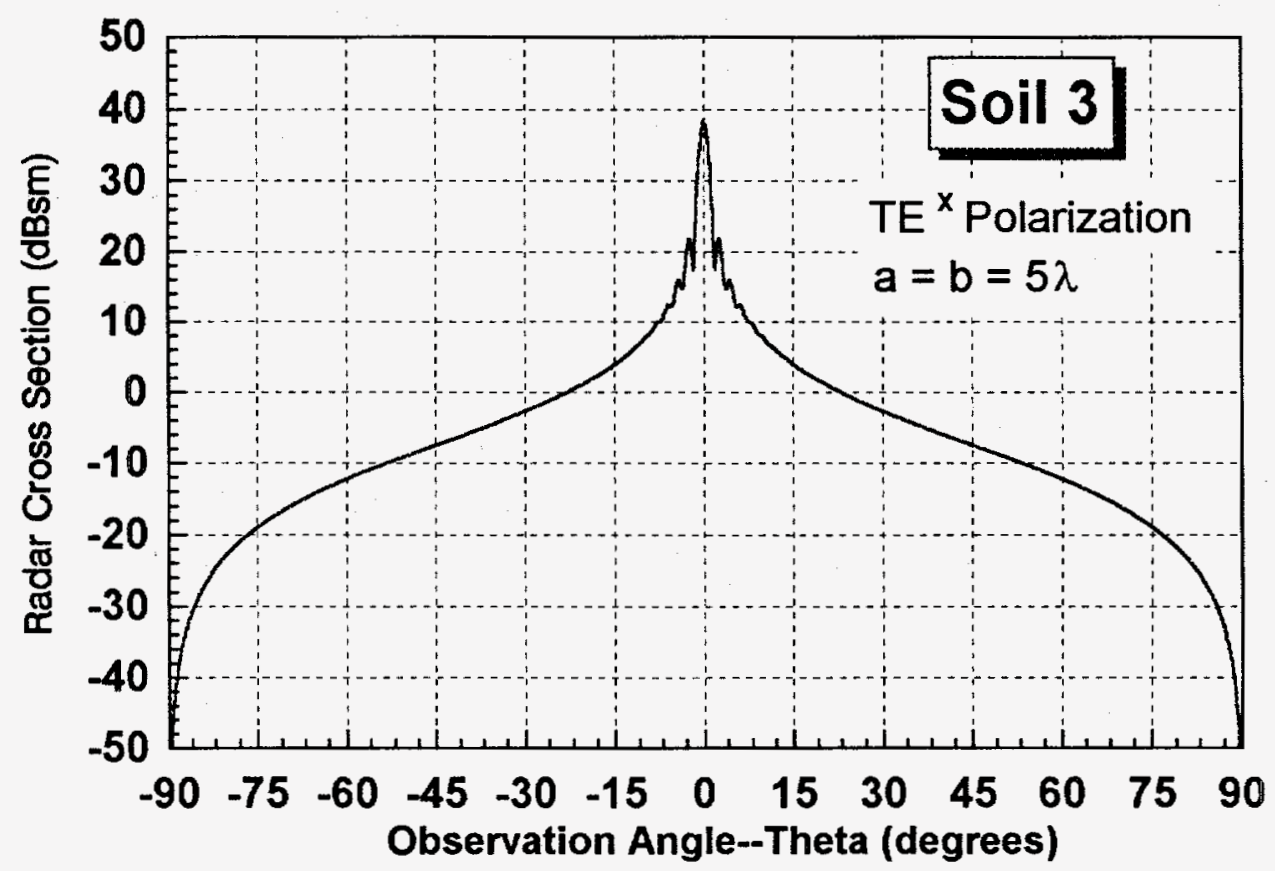

Fig. 14. Principle plane monostatic radar cross section for a $5 \lambda \times 5 \lambda$ rectangular flat plate embedded in San Antonio clay loam with 10\% water.

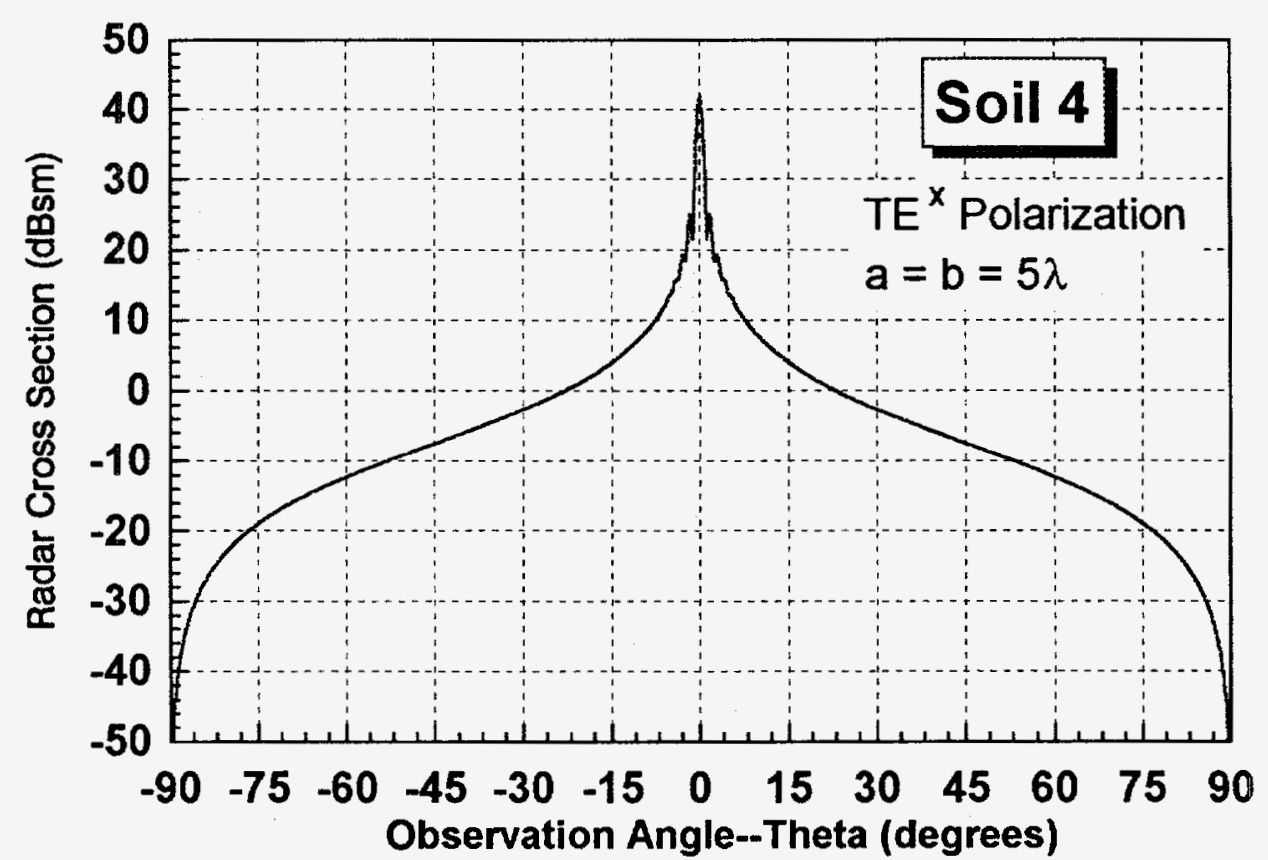

Fig. 15. Principle plane monostatic radar cross section for a $5 \lambda \times 5 \lambda$ rectangular flat plate embedded in San Antonio clay loam with $20 \%$ water. 


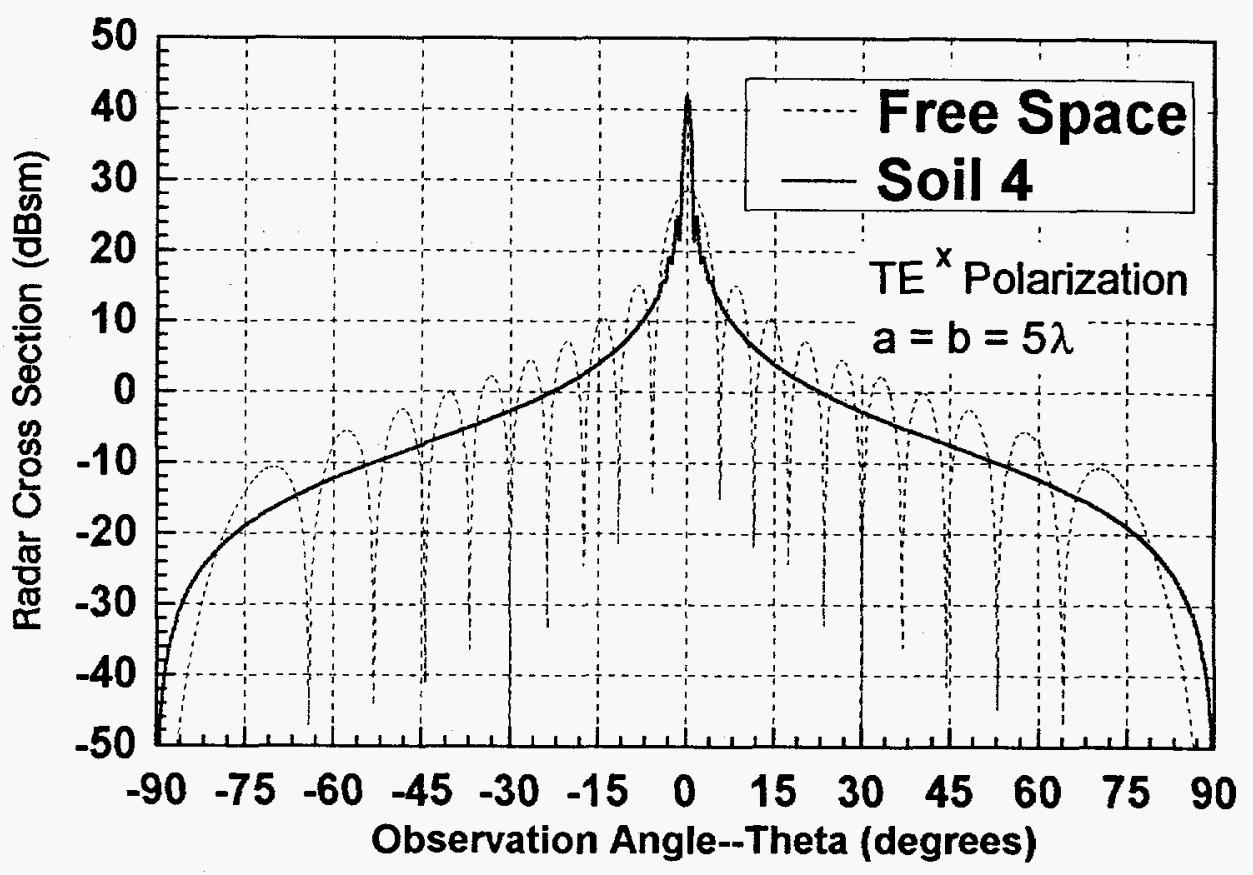

Fig. 16. Comparison of principle plane monostatic radar cross section of a $5 \lambda \times 5 \lambda$ rectangular flat plate embedded in free space vs. San Antonio clay loam with $20 \%$ water.

In the course of examining Fig. $12-16$, it becomes immediately obvious that, counter intuitively, the maximum of the scattered field from the flat plate embedded in the lossy media becomes larger as the loss factor increases, and in all cases is larger than that of the flat plate in free space. This is readily explained, however, by noting that the physical size specified for the flat plate in each simulation was the same, whereas its electrical size increased as the loss factor of the soil increased. That is,

$$
\lambda_{\text {effective }}=\frac{c_{\text {soil }}}{f}=\frac{1}{f \sqrt{\varepsilon \mu}},
$$

where $\varepsilon$ and $\mu$ are the complex constitutive parameters (permittivity and permeability, respectively) of the media in which the plate is embedded, and $f$ is the frequency of operation. It is therefore clear that as the loss factor of the soil increases and $|\varepsilon|$ becomes larger $(\mu$ is assumed in the above cases to be equal to that of free space: $\left.4 \pi \times 10^{-7}\right), \lambda_{\text {effective }}$ becomes smaller, and the electrical size of the flat plate increases. From (67) it is therefore seen that the scattered field for normally incident plane waves will similarly be larger.

The second observation that can be made from the above plots is that the $\sin (x) / x$ pattern that typically characterizes the radar cross section of a flat plate (in free space) versus aspect angle is progressively attenuated for the flat plate embedded in soils $1,2,3$, and 4 , or in other words, as the loss factor of the media increases. This may be understood by observing that the $\sin (x) / x$ pattern is generated by the energy which is scattered from the two edges of the flat plate that are parallel to the $\hat{\mathbf{x}}$-axis interacting in a constructive and destructive manner. That is, as the phasing 
between the diffracted energy from the edges varies in conjunction with the variation in aspect angle of the plate, a pattern of peaks and nulls occurs. In a free space medium, the magnitude of the diffracted energy from each of the edges is essentially equivalent, such that destructive interference produces deep nulls in the RCS pattern. In a lossy medium, however, the diffracted energy from the far edge of the flat plate (with respect to the radar) is significantly attenuated in comparison to the energy scattered from the near edge. Therefore, as the aspect angle of the plate is varied from normal incidence such that the relative distance between the edges increases (with respect to the radar) and the amplitude of the scattered energy from the far edge is significantly reduced with respect to the near edge, the RCS of the plate becomes dominated by the scattering from the near edge, and the interference pattern dampens out.

This phenomena is well-illustrated by Fig. 17-18. Fig. 17 is a 4096-point IFFT of the stepped frequency response $(0.5-2.0 \mathrm{GHz})$ of a rectangular flat plate $(a=b=59.02$ inches $)$ in free space oriented at an angle of $\theta_{i}=\theta_{s}=30^{\circ}$ with respect to the radar. The scattering from the two edges is clearly resolved, and the amplitude of the diffracted energy from each of the edges is seen to be equivalent. Fig. 18 is a 4096-point IFFT of the stepped frequency response $(0.5-2.0 \mathrm{GHz})$ of the same rectangular flat plate ( $a=b=59.02$ inches) oriented at an angle of $\theta_{i}=\theta_{s}=30^{\circ}$ with respect to the radar, but embedded in San Antonio clay loam with 5\% water. Again the scattering from the two edges is clearly resolved; however, the energy diffracted from the far edge of the plate is highly attenuated (by $\sim 40 \mathrm{~dB}$ ) in comparison to that of the near edge. It therefore becomes clear as to why the interference pattern disappears.

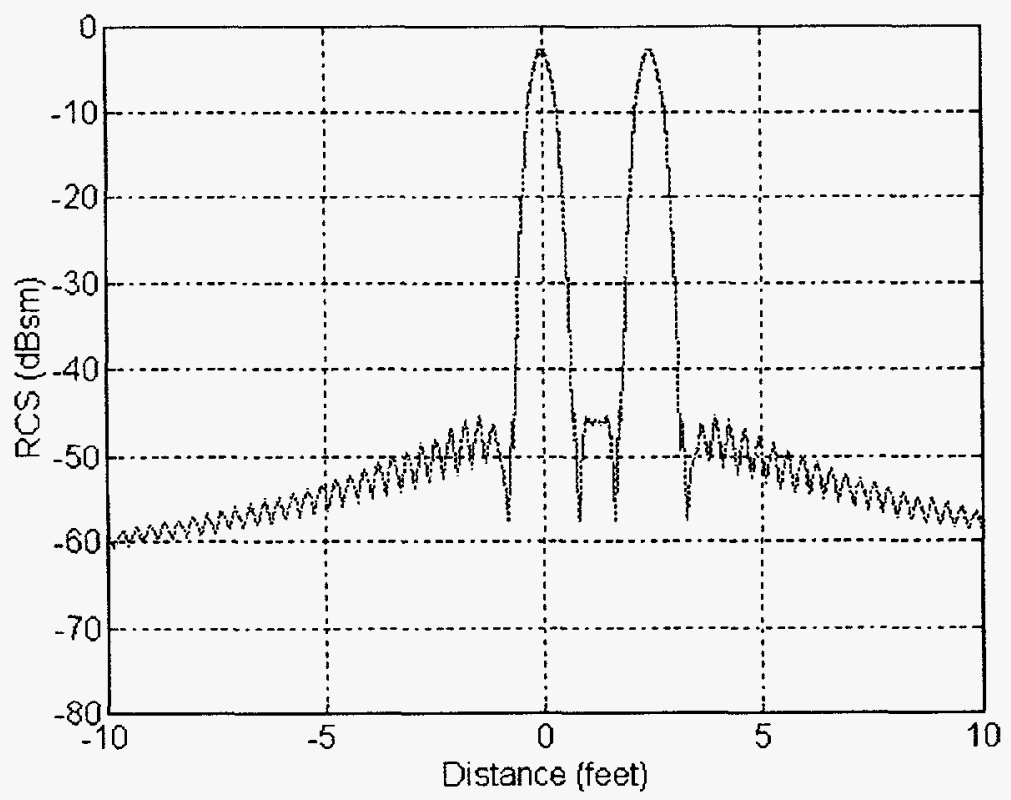

Fig. 17. One-dimensional image of scattering from rectangular flat plate $(a=b=59.02$ inches) located in free space and oriented at angle of $30^{\circ}$ with respect to radar. 


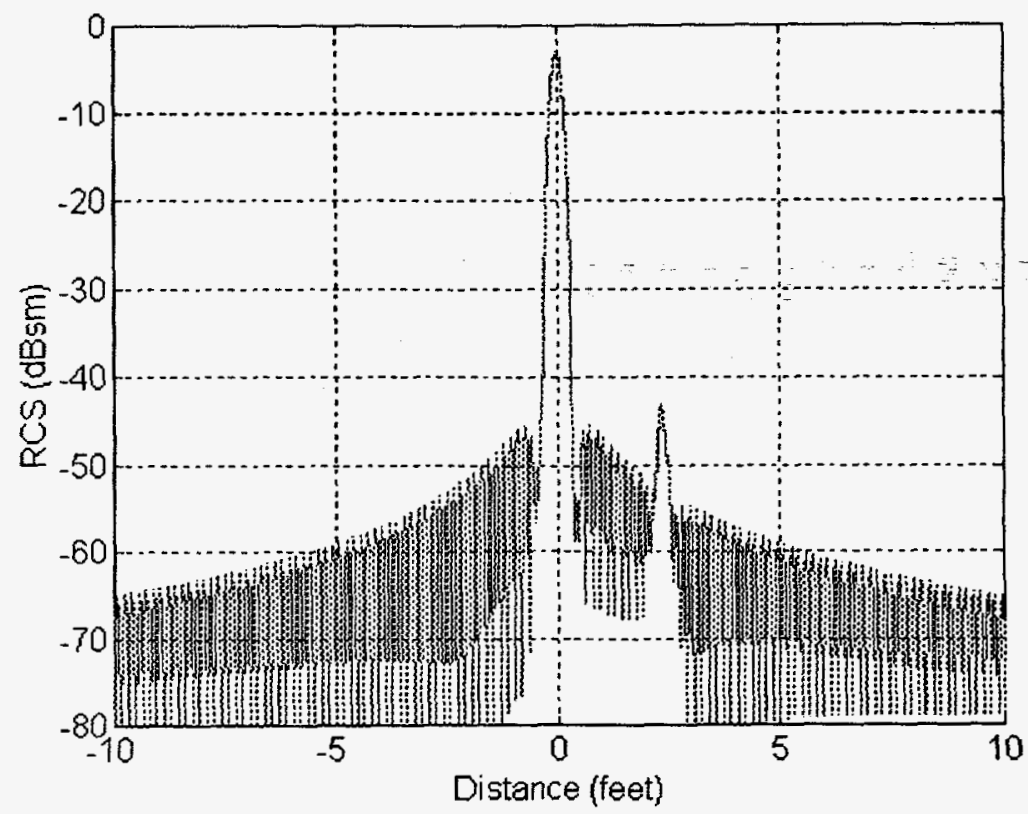

Fig. 18. One-dimensional image of scattering from rectangular flat plate $(a=b=59.02$ inches) embedded in San Antonio clay loam with 5\% water and oriented at angle of $30^{\circ}$ with respect to radar.

As a final observation, the fact that scattering from the far edge of the flat plate is highly attenuated relative to scattering from the near edge of the plate leads one to conclude that the process of attempting to identify objects embedded in a lossy medium by means of a "characteristic" radar cross section signature may be a very difficult task. Since physical objects of even modest complexity are typically composed of a number of scattering centers which are not equidistant in space relative to the illuminating radar, the radar cross section of an object embedded in a lossy medium will change dramatically from its analogous free space value (as has been seen), and the measured value will be highly dependent not only on the orientation of the object, but also on the constitutive parameters of the medium.

\section{Radar Cross Section of a Perfectly Conducting Circular Cylinder Embedded in Lossy Media}

\subsection{TMZ Polarization}

The second object to be considered is scattering from a two-dimensional (infinitely long), perfectly conducting circular cylinder having a radius of $a$ that is illuminated by a uniform plane wave and that is embedded in a lossy medium. Cylinders are an important class of geometrical objects to consider, since they are representative of a wide range of practical scatterers, such as missiles, fuselages, storage tanks, pipelines, etc. The exact solution for scattering from a two dimensional cylinder in free space is well known and can be found in several references. The derivation given here parallels those given in [2] and [9]. 
To begin, it is assumed that a normalized plane wave traveling in the $+\hat{\mathbf{x}}$ direction is incident upon the cylinder as shown in Fig. 19. This plane wave is $\mathrm{TM}^{z}$-polarized, and can be represented as

$$
\overline{\mathbf{E}}^{i}=\hat{\mathbf{z}} E_{0} e^{-j \gamma x},
$$

where the propagation constant $\gamma$ remains as previously defined in (17). In calculating scattering from the cylinder, it is convenient to express this rectilinear form of the incident plane wave in terms of cylindrical wave functions, since the surface of a cylindrical structure is most conveniently defined using cylindrical coordinates. It has been shown $[2,9]$ that the plane wave can in fact be expressed as an infinite sum of cylindrical wave functions, such that

$$
\overrightarrow{\mathbf{E}}^{i}=\hat{\mathbf{z}} E_{0} e^{-j \gamma x}=\hat{\mathbf{z}} E_{0} e^{-j \gamma \rho \cos \phi}=\hat{\mathbf{z}} E_{0} \sum_{n=-\infty}^{\infty} j^{-n} J_{n}(\gamma \rho) e^{j n \phi},
$$

where $J_{n}(\gamma \rho)$ is an $n^{\text {th }}$-order Bessel function of the first kind, and $n$ is an integer.

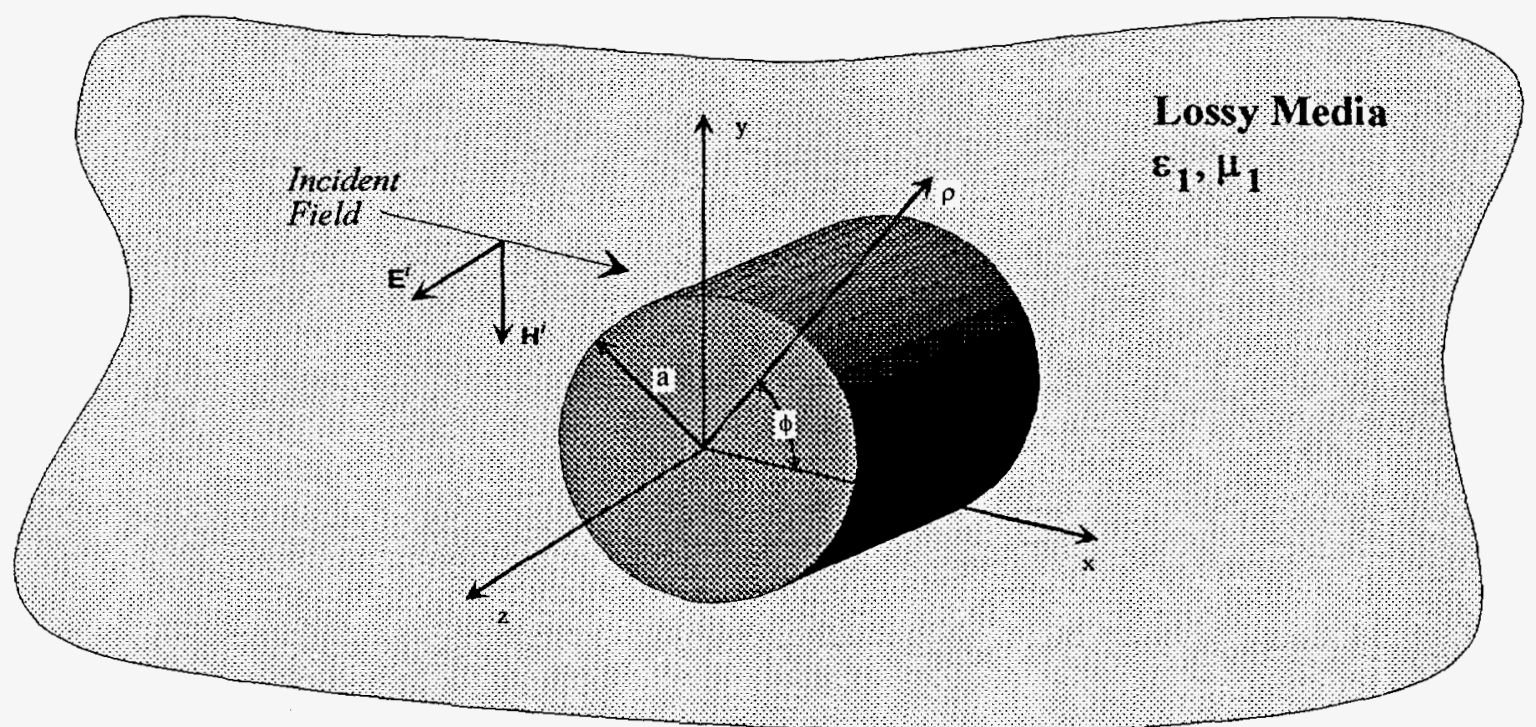

Fig. 19. Uniform plane wave incident on perfectly conducting circular cylinder embedded in lossy media (TM polarization).

The total field that exists with the conducting cylinder present is given by

$$
\overrightarrow{\mathbf{E}}^{t}=\overrightarrow{\mathbf{E}}^{i}+\overrightarrow{\mathbf{E}}^{s},
$$

where $\overline{\mathbf{E}}^{s}$ is the scattered field. Since the scattered field travels in the outward direction away from the cylinder, it can be expressed using the same form as the incident field, but with Hankel functions replacing the Bessel functions, such that

$$
\overline{\mathbf{E}}^{s}=\hat{\mathbf{z}} E_{0} \sum_{n=-\infty}^{\infty} j^{-n} a_{n} H_{n}^{(2)}(\gamma \rho) e^{j n \phi}
$$

in which $a_{n}$ represents the as yet unknown amplitude coefficients of the cylindrical wave functions. These coefficients may be solved for by utilizing the boundary condition that the total 
field at the surface of the cylinder must be equal to zero (since the cylinder has been chosen to be perfectly conducting); that is,

$$
\overrightarrow{\mathbf{E}}^{t}=\hat{\mathbf{z}} E^{t}(\rho=a, 0 \leq \phi \leq 2 \pi)=0 .
$$

Therefore, combining (72), (73), and (74),

$$
\hat{\mathbf{z}} E_{0} \sum_{n=-\infty}^{\infty}\left[j^{-n} e^{j n \phi}\left(J_{n}(\gamma a)+a_{n} H_{n}^{(2)}(\gamma a)\right)\right]=0,
$$

such that

$$
a_{n}=-\frac{J_{n}(\gamma a)}{H_{n}^{(2)}(\gamma a)} .
$$

The scattered field from the cylinder may now be written as

$$
\begin{aligned}
\overrightarrow{\mathbf{E}}^{s} & =-\hat{\mathbf{z}} E_{0} \sum_{n=-\infty}^{\infty} j^{-n} \frac{J_{n}(\gamma a)}{H_{n}^{(2)}(\gamma a)} H_{n}^{(2)}(\gamma \rho) e^{j n \phi} \\
& =-\hat{\mathbf{z}} E_{0} \sum_{n=0}^{\infty}(-j)^{n} \varepsilon_{n} \frac{J_{n}(\gamma a)}{H_{n}^{(2)}(\gamma a)} H_{n}^{(2)}(\gamma \rho) \cos (n \phi)
\end{aligned}
$$

where

$$
\varepsilon_{n}=\left\{\begin{array}{ll}
1 & n=0 \\
2 & n \neq 0
\end{array} .\right.
$$

For the purposes of calculating scattering from the cylinder in the far-field region, the large argument approximation for the Hankel function can be used to reduce the expression for the scattered field. According to [5],

$$
H_{n}^{(2)}\left(z \stackrel{|z| \rightarrow \infty}{\approx} \sqrt{\frac{2}{\pi z}} e^{-3\left(z-\frac{n \pi}{2}-\frac{\pi}{4}\right)},\right.
$$

where $z$ is a complex number. This expression can be rewritten as

$$
H_{n}^{(2)}(z) \stackrel{|z| \rightarrow \infty}{\approx} \sqrt{\frac{2 j}{\pi z}} j^{n} e^{-j z}
$$

such that in the far field, (77) can be expressed as

$$
\overline{\mathbf{E}}^{s} \stackrel{|\gamma \rho| \rightarrow \infty}{\cong}-\hat{\mathbf{z}} E_{0} \sqrt{\frac{2 j}{\pi \gamma \rho}} e^{-j \gamma \rho} \sum_{n=0}^{\infty} \varepsilon_{n} \frac{J_{n}(\gamma a)}{H_{n}^{(2)}(\gamma a)} \cos (n \phi)
$$

For a two-dimensional target, the scattering parameter is typically referred to as the scattering width or radar cross section per unit length, and is conventionally expressed as [2] 


$$
\sigma_{2 d}=\lim _{\rho \rightarrow \infty} 2 \pi \rho \frac{\left|\overrightarrow{\mathbf{E}}^{s}\right|^{2}}{\left|\overrightarrow{\mathbf{E}}^{i}\right|^{2}}=\lim _{\rho \rightarrow \infty} 2 \pi \rho \frac{\left|\overrightarrow{\mathbf{H}}^{s}\right|^{2}}{\left|\overrightarrow{\mathbf{H}}^{i}\right|^{2}} .
$$

However, in order to be consistent with the new definition for the radar cross section for an object embedded in a lossy full space as given in (14) in which the loss along the path from the dominant specular point of the object to the radar is factored out, the above expression for the scattering width must similarly be modified, resulting in

$$
\sigma_{\mu \varepsilon_{2 d}}=\lim _{\rho \rightarrow \infty} 2 \pi \rho \frac{\left|e^{\mid \operatorname{Im}(\gamma)(R-a)} \overline{\mathbf{E}}^{s}\right|^{2}}{\left|e^{|\operatorname{lm}(y)| a}\right|^{2}}=\lim _{\rho \rightarrow \infty} 2 \pi \rho \frac{\left|e^{|\operatorname{Im}(y)|(R-a)} \overline{\mathbf{H}}^{s}\right|^{2}}{\left|e^{\operatorname{Im}(y) \mid a}\right|^{2}},
$$

where as before, $a$ is the distance from the coordinate system origin to the dominant specular point of the object, and the amplitude of the incident wave is normalized to that at the specular point. In the case of the cylinder, the distance to the specular point is $a$, the radius of the cylinder. Substituting the expression for $\overrightarrow{\mathbf{E}}^{s}$ given in (81) into (83) provides the result for the TM $z$ scattering width of the two-dimensional cylinder that

$$
\sigma_{\mu \varepsilon_{2 d}}^{T M^{2}}=4\left|\frac{e^{-2 \alpha|\mathrm{m}(\gamma)|}}{\sqrt{\gamma}} \sum_{n=0}^{\infty} \varepsilon_{n} \frac{J_{n}(\gamma a)}{H_{n}^{(2)}(\gamma a)} \cos (n \phi)\right|^{2} .
$$

As previously noted, for purposes of this study, the interest is in monostatic backscattering; as seen in Fig. 19, the angle $\phi$ is therefore equal to $180^{\circ}$, and the expression above can finally be written as

$$
\sigma_{\mu \varepsilon_{2 d}}^{T M^{z}}=4\left|\frac{e^{-2 a \mid \mathrm{m}(\gamma) \|}}{\sqrt{\gamma}} \sum_{n=0}^{\infty}(-1)^{n} \varepsilon_{n} \frac{J_{n}(\gamma a)}{H_{n}^{(2)}(\gamma a)}\right|^{2},
$$

where $\varepsilon_{n}$ is as given in (78).

\subsection{TEZ Polarization}

The second problem of interest with regard to the cylinder is scattering when a $\mathrm{TE}^{z}$-polarized plane wave is normally incident upon it as shown in Fig. 20. Again, the cylinder is assumed to be a two-dimensional (infinitely long), perfectly conducting circular cylinder having a radius of $a$, and is embedded in a lossy (full space) medium. It is most convenient in solving this problem to use the magnetic field to find the scattering width of the cylinder. Proceeding as before, then, the normalized plane wave is assumed to be traveling in the $+\hat{\mathbf{x}}$ direction, and can be represented as

$$
\overrightarrow{\mathbf{H}}^{i}=\hat{\mathbf{z}} H_{0} e^{-j \hat{\gamma} x}=\hat{\mathbf{z}} H_{0} e^{-j \gamma \rho \cos \phi}=\hat{\mathbf{z}} H_{0} \sum_{n=-\infty}^{\infty} j^{-n} J_{n}(\gamma \rho) e^{j n \phi} .
$$

Again, the total field that exists with the cylinder present is the sum of the incident and scattered fields, such that

$$
\overrightarrow{\mathbf{H}}^{t}=\overrightarrow{\mathbf{H}}^{i}+\overrightarrow{\mathbf{H}}^{s},
$$


where $\overrightarrow{\mathbf{H}}^{s}$ is the scattered field. The scattered field can be expressed using the same form as the incident field with Hankel functions replacing the Bessel functions (since the scattered field is traveling outward away from the cylinder), such that

$$
\overline{\mathbf{H}}^{s}=\hat{\mathbf{z}} H_{0} \sum_{n=-\infty}^{\infty} j^{-n} b_{n} H_{n}^{(2)}(\gamma \rho) e^{j n \phi},
$$

where $b_{n}$ are the presently unknown amplitude coefficients of the cylindrical wave functions. The total magnetic field is therefore given by

$$
\overrightarrow{\mathbf{H}}^{t}=\hat{\mathbf{z}} H_{0} \sum_{n=-\infty}^{\infty}\left[j^{-n} e^{j n \phi}\left(J_{n}(\gamma \rho)+b_{n} H_{n}^{(2)}(\gamma \rho)\right)\right] .
$$

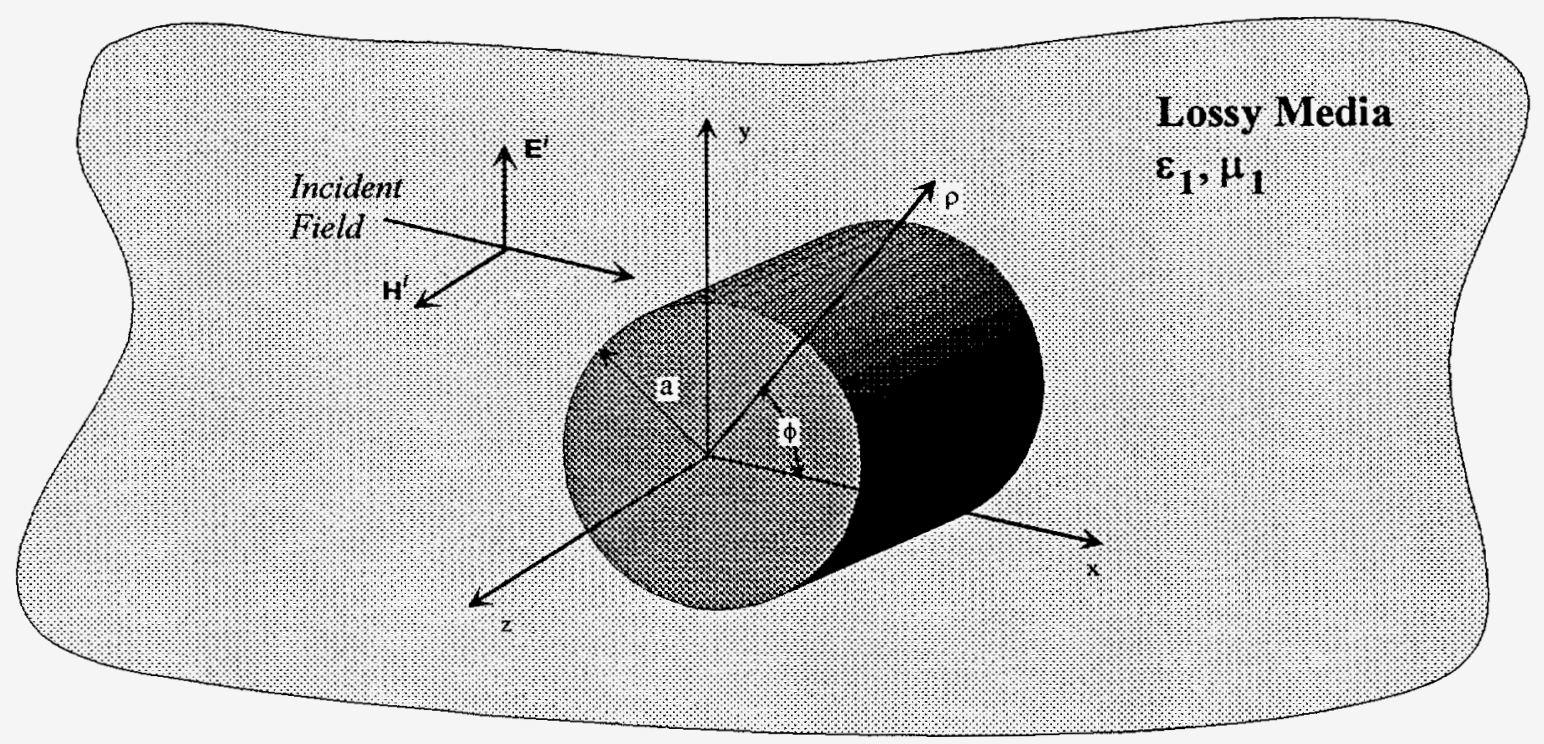

Fig. 20. Uniform plane wave incident on perfectly conducting circular cylinder embedded in lossy media ( $\mathrm{TE}^{z}$ polarization).

The boundary condition that may be utilized to solve for the coefficients $b_{n}$ is that the tangential component of the total electric field must go to zero at the surface of the cylinder since the cylinder is a perfect conductor. That is,

$$
\hat{\phi} E^{t}(\rho=a, 0 \leq \phi \leq 2 \pi)=0 .
$$

The electric field can be found using Maxwell's Ampere equation,

$$
\overline{\mathbf{E}}^{t}=\frac{1}{j \omega \varepsilon} \nabla \times \overline{\mathbf{H}}^{t},
$$

which in this instance reduces to

$$
\overline{\mathbf{E}}^{t}=\frac{1}{j \omega \varepsilon}\left[\hat{\boldsymbol{\rho}} \frac{1}{\rho} \frac{\partial \overline{\mathbf{H}}^{t}}{\partial \phi}-\hat{\phi} \frac{\partial \overrightarrow{\mathbf{H}}^{t}}{\partial \rho}\right]
$$


since $\overrightarrow{\mathbf{H}}^{t}$ is composed solely of $\hat{\mathbf{z}}$ - polarized components. Solving, then, for the $\hat{\phi}$ - polarized component of the total $\overline{\mathbf{E}}$ field results in

$$
E_{\phi}^{t}=j \frac{\gamma H_{0}}{\omega \varepsilon} \sum_{n=-\infty}^{\infty}\left[j^{-n} e^{j n \phi}\left(J_{n}^{\prime}(\gamma \rho)+b_{n} H_{n}^{(2 \gamma}(\gamma \rho)\right)\right],
$$

where the ' sign indicates a partial derivative with respect to the argument of the Bessel or Hankel function. Combining (90) and (93),

$$
j \frac{\gamma H_{0}}{\omega \varepsilon} \sum_{n=-\infty}^{\infty}\left[j^{-n} e^{j n \phi}\left(J_{n}^{\prime}(\gamma a)+b_{n} H_{n}^{(2 \gamma}(\gamma a)\right)\right]=0,
$$

leading to the result that

$$
b_{n}=-\frac{J_{n}^{\prime}(\gamma a)}{H_{n}^{(2)}(\gamma a)} .
$$

The scattered magnetic field from the cylinder may therefore now be written as

$$
\begin{aligned}
\overrightarrow{\mathbf{H}}^{s} & =-\hat{\mathbf{z}} H_{0} \sum_{n=-\infty}^{\infty} j^{-n} \frac{J_{n}^{\prime}(\gamma a)}{H_{n}^{(2)}(\gamma a)} H_{n}^{(2)}(\gamma \rho) e^{j n \phi} \\
& =-\hat{\mathbf{z}} H_{0} \sum_{n=0}^{\infty}(-j)^{n} \varepsilon_{n} \frac{J_{n}^{\prime}(\gamma a)}{H_{n}^{(2)}(\gamma a)} H_{n}^{(2)}(\gamma \rho) \cos (n \phi)
\end{aligned},
$$

where $\varepsilon_{n}$ is as given in (78).

The large argument approximation for the Hankel function as given in (80) can again be used to reduce the expression for the scattered field in the far-field region, such that

$$
\overline{\mathbf{H}}^{s} \stackrel{|\gamma p| \rightarrow \infty}{\cong}-\hat{\mathbf{z}} H_{0} \sqrt{\frac{2 j}{\pi \gamma \rho}} e^{-j \gamma \rho} \sum_{n=0}^{\infty} \varepsilon_{n} \frac{J_{n}^{\prime}(\gamma a)}{H_{n}^{(2)}(\gamma a)} \cos (n \phi) .
$$

Using this expression for $\overrightarrow{\mathbf{H}}^{s}$ in the modified definition of the scattering width as given in (83), and again noting that $\phi=180^{\circ}$ for monostatic backscattering, the scattering width for a twodimensional cylinder having a normally-incident $\mathrm{TE}^{z}$-polarized plane wave can thus be written as

$$
\sigma_{\mu \varepsilon_{2 d}}^{T E^{2}}=4\left|\frac{e^{-2 a|\operatorname{m}(\gamma)|}}{\sqrt{\gamma}} \sum_{n=0}^{\infty}(-1)^{n} \varepsilon_{n} \frac{J_{n}^{\prime}(\gamma a)}{H_{n}^{(2)}(\gamma a)}\right|^{2} .
$$

\subsection{Calculated Radar Cross Section for Buried Cylinders}

Fig. $21-24$ show the calculated scattering width as a function of frequency for a twodimensional, perfectly conducting cylinder of infinite length having a radius of $0.5 \mathrm{~m}$. The cylinder is embedded in the same lossy soil types as used in the flat plate calculations (as defined in Table 1). Fig. 25 then compares the calculated scattering width for the same cylinder located in a lossless medium (free space) with the calculated scattering width of the cylinder embedded in San Antonio clay loam with $20 \%$ water. 


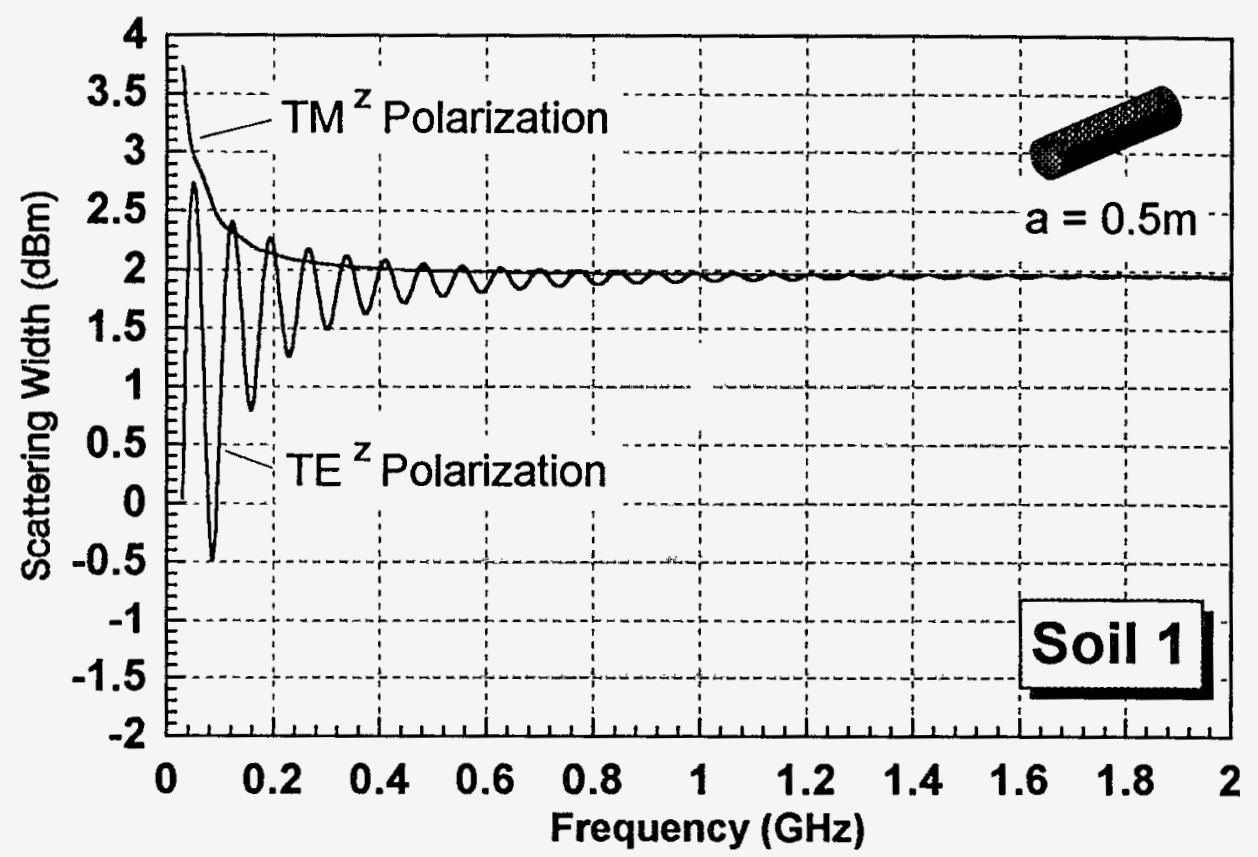

Fig. 21. Monostatic scattering width for a two-dimensional cylinder of radius 0.5 meters embedded in dry sand.

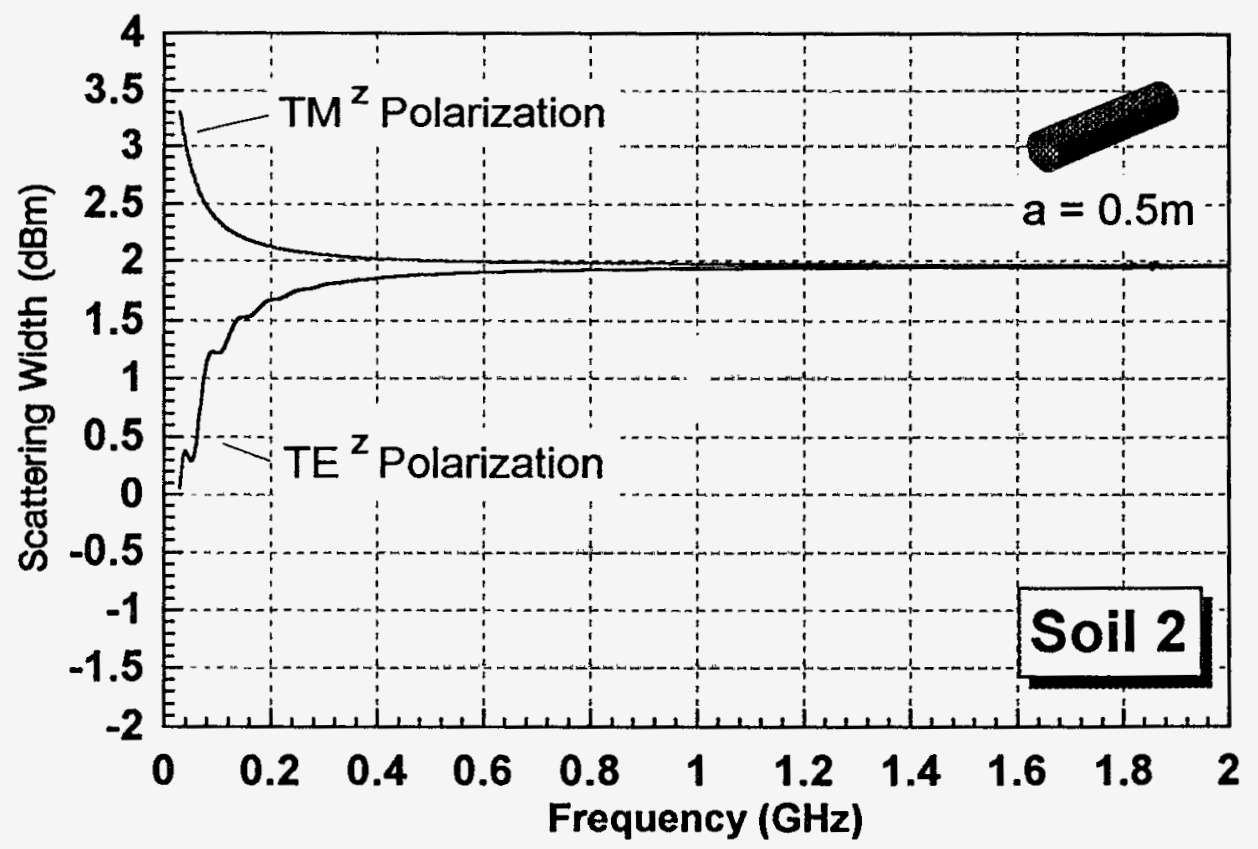

Fig. 22. Monostatic scattering width for a two-dimensional cylinder of radius 0.5 meters embedded in San Antonio clay loam with 5\% water. 


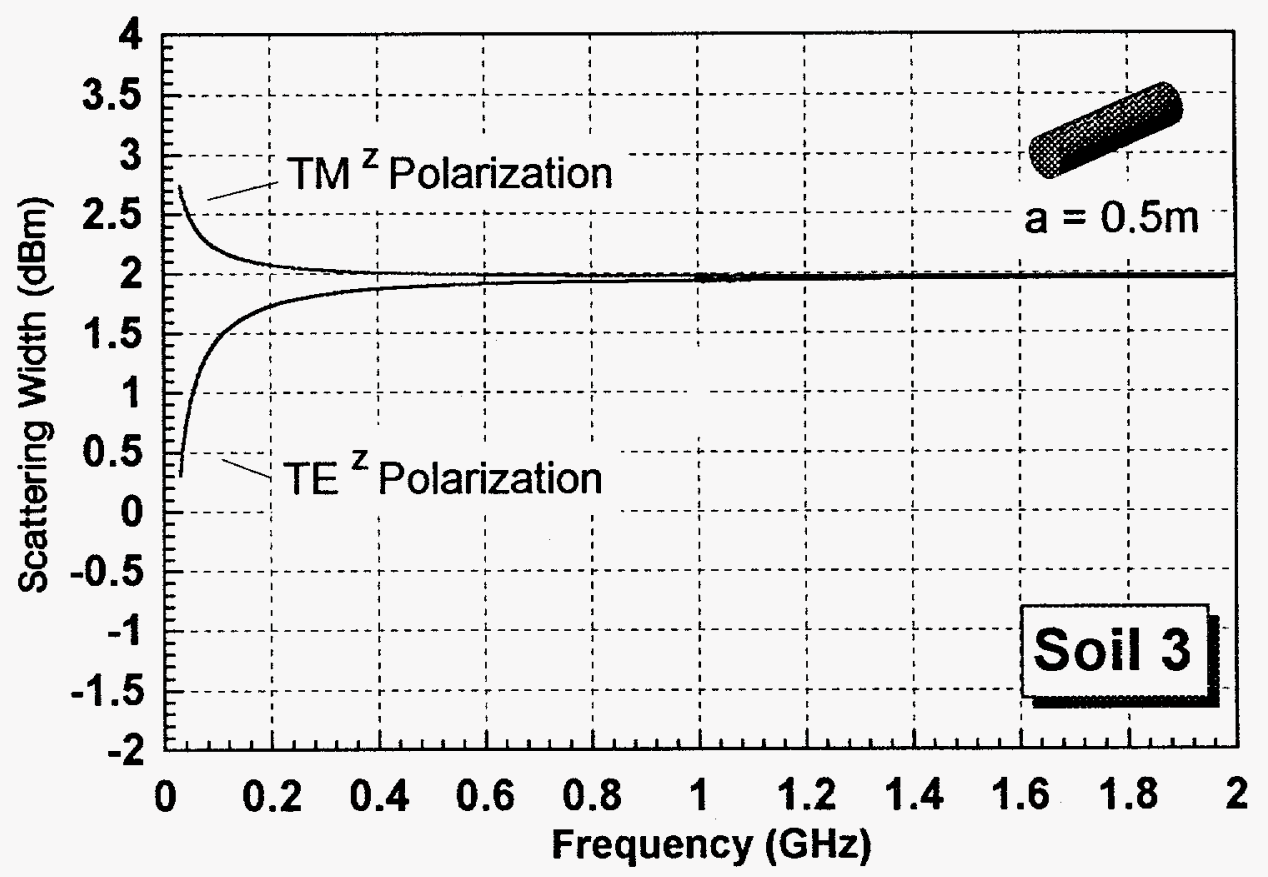

Fig. 23. Monostatic scattering width for a two-dimensional cylinder of radius 0.5 meters embedded in San Antonio clay loam with 10\% water.

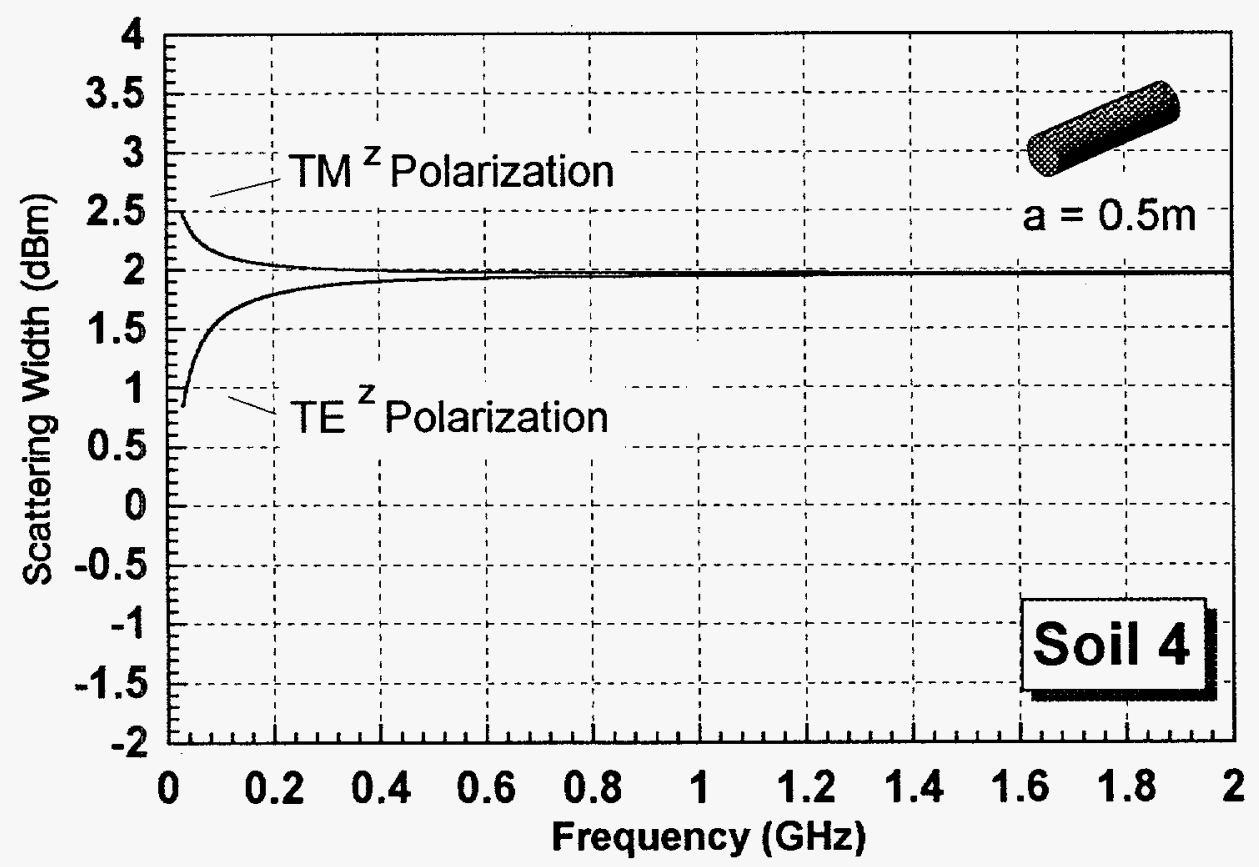

Fig. 24. Monostatic scattering width for a two-dimensional cylinder of radius 0.5 meters embedded in San Antonio clay loam with $20 \%$ water. 


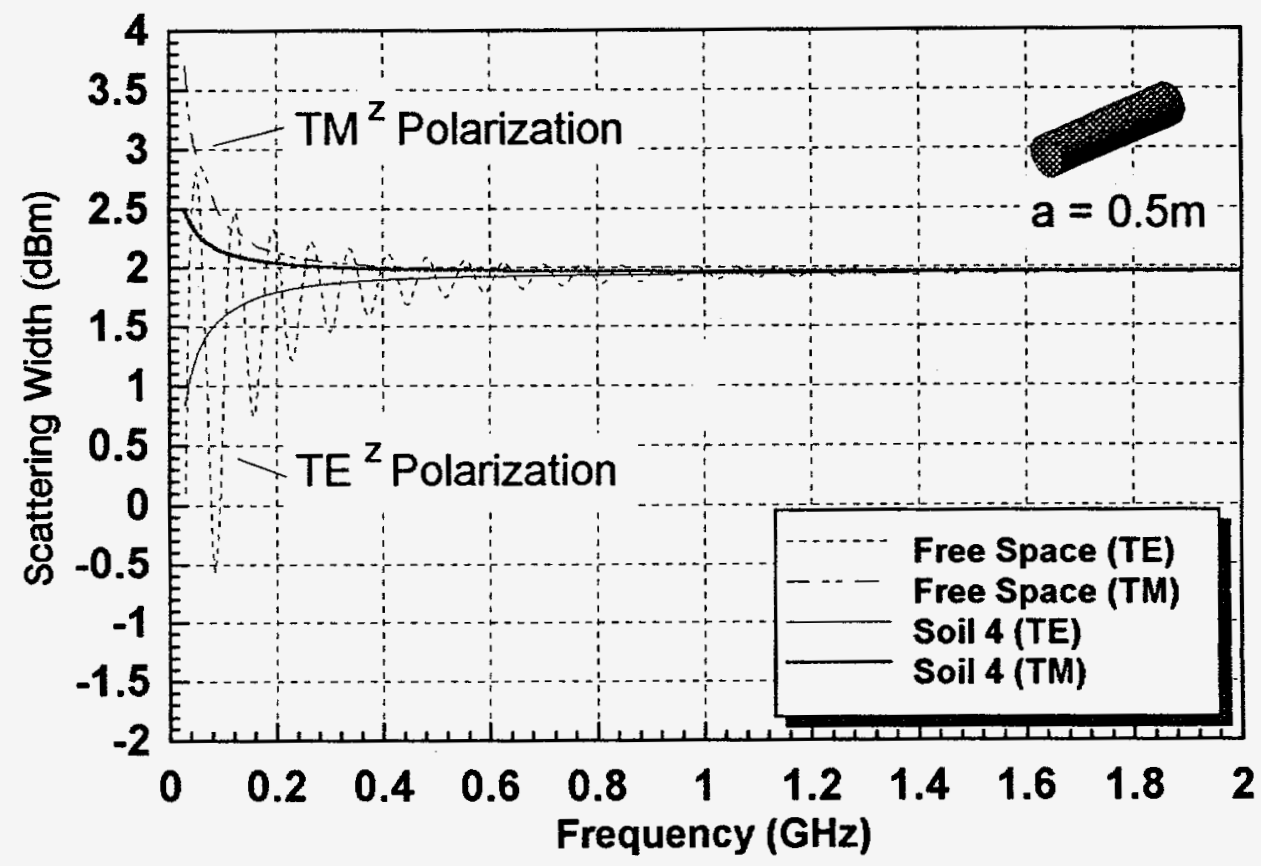

Fig. 25. Comparison of monostatic scattering widths for a two-dimensional cylinder of radius 0.5 meters embedded in free space vs. San Antonio clay loam with $20 \%$ water.

It can be seen from Fig. 21 and 25 that the $\mathrm{TE}^{z}$-polarized scattering width of the cylinder located in free space or in a relatively low-loss medium displays an oscillatory behavior at the lower frequencies; the oscillations dampen out with increasing frequency, approaching a constant value of $\pi a$ as the wavelength becomes small with respect to the size of the cylinder. The oscillations are caused by a creeping wave that is excited by the $\mathrm{TE}^{2}$-polarized incident field which travels around the rear of the cylinder and which therefore varies in and out of phase with the specular scattering from the near side of the cylinder as the frequency changes. The contribution from the creeping wave to the scattering width becomes progressively smaller as the electrical size of the cylinder increases (due to the decreasing wavelength), such that the oscillations are attenuated as the frequency is increased.

More importantly with regard to this study, these figures show that the oscillations are also severely attenuated as the loss factor of the medium in which the cylinder is embedded increases. For instance, the oscillations are barely discernible for the cylinder embedded in San Antonio clay loam with 5\% water (Fig. 22), and have completely disappeared for the cylinder located in San Antonio clay loam with $10 \%$ and $20 \%$ water. The reason for the dampening of the oscillatory behavior is that the creeping wave return from the cylinder in a lossy medium is significantly attenuated in comparison to the specular backscattered energy, largely because the creeping wave must traverse a longer distance in the lossy medium (with respect to the radar) than does the specular return. As the loss factor of the medium in which the cylinder is embedded is increased, the amplitude of the creeping wave is increasingly attenuated, such that the scattering becomes completely dominated by the specular return; hence, the oscillations vanish. 
These results further substantiate the conclusion previously suggested that the process of attempting to identify objects embedded in a lossy medium by means of a "characteristic" radar cross section signature may be a very difficult task, due to the fact that scattering from an object embedded in a lossy medium changes dramatically from its analogous free space value, dependent not only on the orientation of the object with respect to the incident field, but also on the constitutive parameters of the medium.

\section{Radar Cross Section of a Perfectly Conducting Sphere Embedded in Lossy Media}

The exact solution for the field scattered from a conducting sphere in free space was obtained in the early part of this century by Mie, and is quite well known. The solution is also valid when the media has electric permittivity and magnetic permeability different from free space. For completeness, the solution is summarized here. The geometry is described in Fig. 26. Assuming a time dependence of $e^{j \omega t}$, and using the vector spherical harmonics as described in Jackson [4], the scattered field from a sphere of radius $a$ can be written

$$
\overrightarrow{\mathbf{E}}_{s}=\frac{1}{2} \sum_{p=1}^{\infty} j^{p} \sqrt{4 \pi(2 p+1)}\left[\alpha_{ \pm}(p) h_{p}^{(2)}(k r) \overrightarrow{\mathbf{X}}_{p, \pm 1} \pm \frac{\beta_{ \pm}(p)}{k} \nabla \times h_{p}^{(2)}(k r) \overrightarrow{\mathbf{X}}_{p, \pm 1}\right]
$$

where the coefficients $\alpha_{ \pm}(p)$ and $\beta_{ \pm}(p)$ are obtained by forcing the continuity of the tangential electric field intensity and the normal magnetic flux density at the surface of the sphere. This results in

$$
\begin{gathered}
\alpha_{ \pm}(p) \equiv-\left[\frac{h_{p}^{(1)}(k a)}{h_{p}^{(2)}(k a)}+1\right], \\
\beta_{ \pm}(p) \equiv-\left[\left.\frac{\frac{d}{d r}\left[r h_{p}^{(1)}(k r)\right]}{\frac{d}{d r}\left[r h_{p}^{(2)}(k r)\right]}\right|_{r=a}\right]+1 .
\end{gathered}
$$

The spherical Hankel's functions are [5]

$$
h_{p}^{(1)}(z)=\frac{(-j)^{p+1}}{z} e^{j z} \sum_{q=0}^{p} \frac{(p+q) !}{q !(p-q) !} \frac{1}{(-2 j z)^{q}},
$$

and

$$
h_{p}^{(2)}(z)=\frac{(j)^{p+1}}{z} e^{-j z} \sum_{q=0}^{p} \frac{(p+q) !}{q !(p-q) !} \frac{1}{(2 j z)^{q}} .
$$

$\overrightarrow{\mathbf{X}}_{p, \pm 1}$ is the vector spherical harmonic given by

$$
\overline{\mathbf{X}}_{p, m}(\theta, \phi)=\frac{1}{\sqrt{p(p+1)}} \overrightarrow{\mathbf{L}} Y_{p, m}(\theta, \phi)
$$


where $\overrightarrow{\mathbf{L}}$ is called the angular momentum operator,

$$
\overrightarrow{\mathbf{L}}=-j \overrightarrow{\mathbf{r}} \times \nabla=j \hat{\theta} \frac{1}{\sin \theta} \frac{\partial}{\partial \phi}-j \hat{\phi} \frac{\partial}{\partial \theta},
$$

and the scalar spherical harmonics are

$$
Y_{p, m}(\theta, \phi)=\sqrt{\frac{2 p+1}{4 \pi} \frac{(p-m) !}{(p+m) !}} P_{p}^{m}(\cos \theta) e^{j m \phi}=(-1)^{m} Y_{p,-m}^{*}(\theta, \phi)
$$

with $P_{p}^{m}(x)$ the associated Legendre function of order $p$ and degree $m$ [4]

$$
P_{p}^{m}(x)=\frac{(-1)^{m}}{2^{p} p !}\left(1-x^{2}\right)^{m / 2} \frac{d^{p+m}}{d x^{p+m}}\left(x^{2}-1\right)^{p} .
$$

The \pm refers to the two senses of circular polarization as the incident field is assumed to be a circularly polarized plane wave incident along the $\hat{\mathbf{z}}$-axis, given by [4]

$$
\overrightarrow{\mathbf{E}}_{i}=\frac{(\hat{\mathbf{x}} \pm j \hat{\mathbf{y}})}{\sqrt{2}} e^{j k z}=\sum_{p=1}^{\infty} j^{p} \sqrt{4 \pi(2 p+1)}\left[j_{p}(k z) \overrightarrow{\mathbf{X}}_{p, \pm 1} \pm \frac{1}{k} \nabla \times j_{p}(k z) \overrightarrow{\mathbf{X}}_{p, \pm 1}\right]
$$

Note that, when the media is lossy, the incident plane wave has unity amplitude at the origin, but not at the specular point, $z=a$ ( $k$ is chosen to have a negative imaginary part, indicating loss in the direction of propagation).

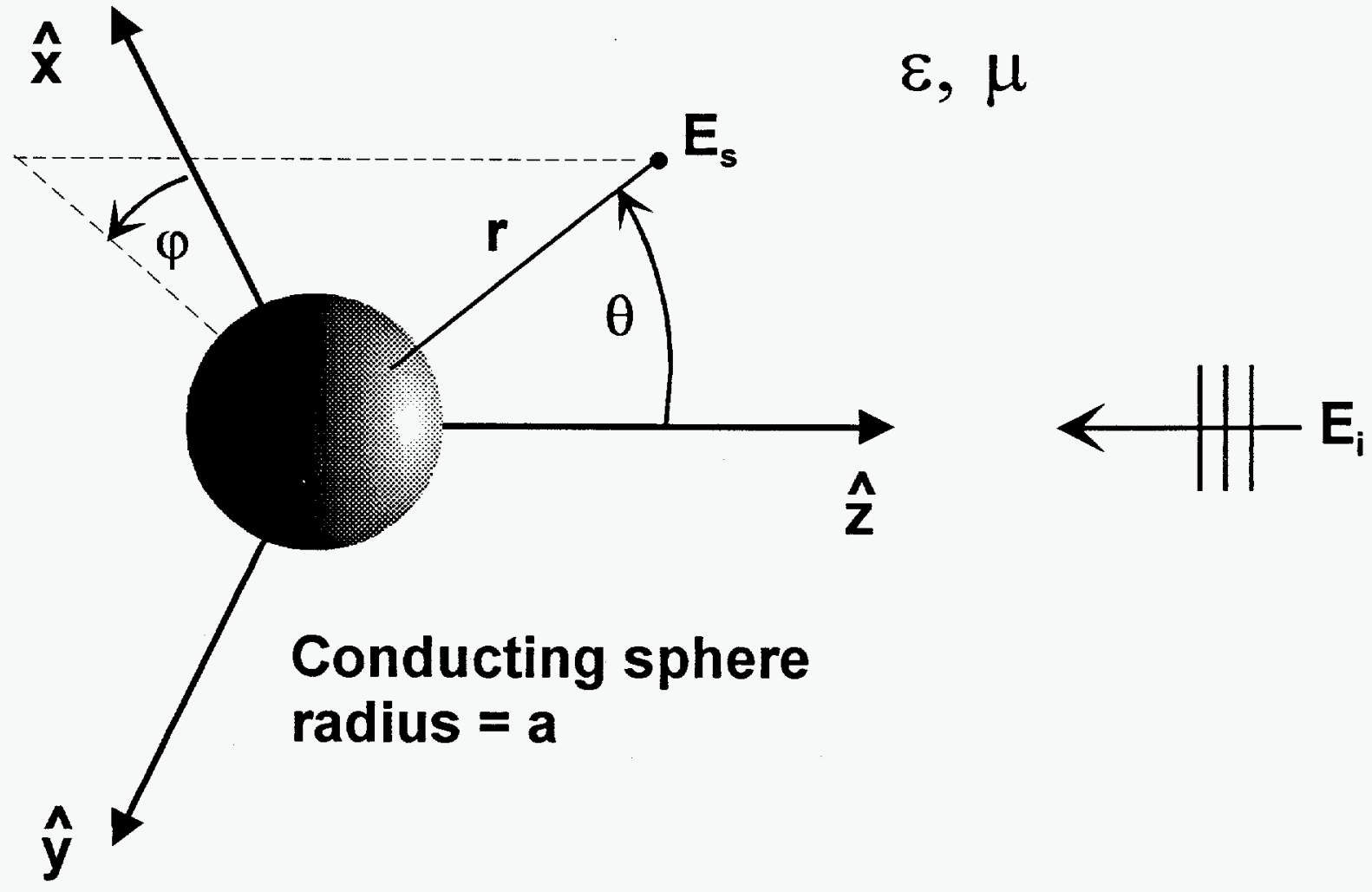

Fig. 26 Plane wave incident on a conducting sphere with radius $a$. 


\subsection{The Angular Momentum Operator and Vector Spherical Harmonics}

In evaluating the vector spherical harmonics, it is useful to represent the angular momentum operator (105) as [4]

$$
\begin{aligned}
\overrightarrow{\mathbf{L}} & =\hat{\mathbf{x}} L_{x}+\hat{\mathbf{y}} L_{y}+\hat{\mathbf{z}} L_{z} \\
& =\hat{\mathbf{x}} \frac{L_{+}+L_{-}}{2}+\hat{\mathbf{y}} \frac{L_{+}-L_{-}}{2 j}+\hat{\mathbf{z}} L_{z},
\end{aligned}
$$

where the operators $L_{x}, L_{y}$, and $L_{z}$ have useful properties and are given by

$$
\begin{gathered}
L_{+}=e^{j \phi}\left(\frac{\partial}{\partial \theta}+\frac{j}{\tan \theta} \frac{\partial}{\partial \phi}\right), \\
L_{-}=e^{-j \phi}\left(-\frac{\partial}{\partial \theta}+\frac{j}{\tan \theta} \frac{\partial}{\partial \phi}\right),
\end{gathered}
$$

and

$$
L_{z}=-j \frac{\partial}{\partial \phi} .
$$

When operating on the spherical harmonics, these operators have the simple properties

$$
\begin{aligned}
& L_{+} Y_{p, m}(\theta, \phi)=\sqrt{(p-m)(p+m+1)} Y_{p, m+1}(\theta, \phi), \\
& L_{-} Y_{p, m}(\theta, \phi)=\sqrt{(p+m)(p-m+1)} Y_{p, m-1}(\theta, \phi),
\end{aligned}
$$

and

$$
L_{z} Y_{p, m}(\theta, \phi)=m Y_{p, m}(\theta, \phi) .
$$

Thus, the vector spherical harmonics become

$$
\begin{aligned}
\overline{\mathbf{X}}_{p, m}(\theta, \phi) & =\frac{1}{2(p(p+1))}\left[\left(\sqrt{(p-m)(p+m+1)} Y_{p, m+1}+\sqrt{(p+m)(p-m+1)} Y_{p, m-1}\right)\right] \hat{\mathbf{x}} \\
& -j \frac{1}{2(p(p+1))}\left[\left(\sqrt{(p-m)(p+m+1)} Y_{p, m+1}-\sqrt{(p+m)(p-m+1)} Y_{p, m-1}\right)\right] \hat{\mathbf{y}} \\
& +\left[2 m Y_{p, m}\right] \hat{\mathbf{z}}
\end{aligned}
$$

A useful recursion relation can be obtained for the scalar spherical harmonics using the recursion relations for the associated Legendre functions [5]

$$
\begin{aligned}
Y_{p+1, m}(\theta, \phi)= & \frac{1}{(p-m+1)} \sqrt{\frac{(2 p+3)(p-m+1) !}{(p+m+1) !}} . \\
& \left\{(2 p+1) \sqrt{\frac{(p+m) !}{(2 p+1)(p-m) !}} \cos \theta Y_{p, m}-(p+m) \sqrt{\frac{(p+m-1) !}{(2 p-1)(p-m-1) !}} Y_{p-1, m}\right\}
\end{aligned}
$$


The first few scalar spherical harmonics are tabulated in [4] and [6].

\subsection{Back-scatter Cross Section of Spheres in a Lossy Media}

The case of interest is the back-scatter case where $\theta=\phi=0$. Since

$$
\left.Y_{1, \pm 1}\right|_{\theta=0}=\left.Y_{2, \pm 1}\right|_{\theta=0}=\left.Y_{2, \pm 2}\right|_{\theta=0}=\left.Y_{3, \pm 2}\right|_{\theta=0}=0,
$$

the recursion relation (117) shows that

$$
\left.Y_{p, \pm 1}\right|_{\theta=0}=0
$$

and

$$
\left.Y_{p, \pm 2}\right|_{\theta=0}=0,
$$

for all $p$. The vector spherical harmonic becomes

$$
\overrightarrow{\mathbf{X}}_{p, \pm 1}(\theta=0, \phi)=\frac{1}{2} Y_{p, 0}(\theta=0, \phi)(\hat{\mathbf{x}} \pm j \hat{\mathbf{y}})=\frac{1}{2} \sqrt{\frac{(2 p+1)}{4 \pi}}(\hat{\mathbf{x}} \pm j \hat{\mathbf{y}})
$$

The scattered field given by (99) simplifies to

$$
\overrightarrow{\mathbf{E}}_{s}=\sum_{p=1}^{\infty} j^{p} \frac{(2 p+1)}{4}\left[\alpha_{ \pm}(p) h_{p}^{(2)}(k r)-j \frac{\beta_{ \pm}(p)}{k r} \frac{\partial}{\partial r}\left(r h_{p}^{(2)}(k r)\right)\right](\hat{\mathbf{x}} \pm j \hat{\mathbf{y}})
$$

where we have also used the fact that

$$
\nabla \times\left(h_{p}^{(2)}(k r) \overrightarrow{\mathbf{X}}_{p, m}(\theta, \phi)\right)=\left[\frac{j h_{p}^{(2)}(k r)}{r} \sqrt{p(p+1)} Y_{p, m}(\theta, \phi)\right] \hat{\mathbf{r}}+\frac{1}{r} \frac{\partial}{\partial r}\left(r h_{p}^{(2)}(k r)\right) \hat{\mathbf{r}} \times \overline{\mathbf{X}}_{p, m}(\theta, \phi),
$$

and the interesting result that

$$
\hat{\mathbf{r}} \times\left.\overrightarrow{\mathbf{X}}_{p, \pm 1}(\theta, \phi)\right|_{\theta=0}=\left.\mp j \overline{\mathbf{X}}_{p, \pm 1}(\theta, \phi)\right|_{\theta=0} .
$$

The back-scatter radar cross section for the conducting sphere (using the definition (14)) is

$$
\sigma_{\mu \varepsilon}=\frac{\pi}{k^{2}} e^{-4|\operatorname{Im}(k)| a}\left|\sum_{p=1}^{\infty}(-1)^{p} \frac{2 p+1}{2}\left[\alpha_{ \pm}(p)-\beta_{ \pm}(p)\right]\right|^{2} \text {. }
$$

\subsection{Behavior of Large Spheres in Lossy Media}

The value of the new definition for radar cross section (14) can be appreciated by examining the behavior of the series for the scattered field for large spheres. To this end, it is useful to examine the coefficients, $\alpha_{ \pm}(p)$ and $\beta_{ \pm}(p)$, in (99). Using the series expressions, (102) and (103), for the spherical Hankel's functions,

$$
\alpha_{ \pm}(p)=(-1)^{p} e^{2 j k a} \frac{\sum_{q=0}^{p} \frac{(p+q) !}{q !(p-q) !(-2 j k a)^{q}}}{\sum_{q=0}^{p} \frac{(p+q) !}{q !(p-q) !} \frac{1}{(2 j k a)^{q}}}-1,
$$


and

$$
\beta_{ \pm}(p)=(-1)^{p} e^{2 j k a} \frac{\sum_{q=0}^{p} \frac{(p+q) !}{q !(p-q) !}\left(1+j \frac{q}{k a}\right) \frac{1}{(-2 j k a)^{q}}}{\sum_{q=0}^{p} \frac{(p+q) !}{q !(p-q) !}\left(1-j \frac{q}{k a}\right) \frac{1}{(2 j k a)^{q}}}-1 .
$$

The point to note is that for large spheres in lossy media, the coefficients grow as

$$
\sim e^{2|\operatorname{lm}(k)| a} \text {. }
$$

By normalizing the radar cross section as described in (14), the loss path is measured to the specular point of the sphere, and the radar cross section is consistent with the free-space radar cross section. Thus, the growth in the coefficients is canceled in the expression (124) for the radar cross section $\sigma_{\mu \varepsilon}$.

The radar cross section behaves in the way intuition would predict, as will be demonstrated in the examples which follow. Because of the loss, the creeping wave is attenuated, and the specular return remains fairly constant with frequency.

\subsection{Calculated Radar Cross Section for Buried Spheres}

The monostatic radar cross section of a perfectly conduction sphere with a $1 \mathrm{~m}$ diameter embedded in dry sand is illustrated in Fig. 27. Since the dry sand is not very lossy, evidence of the creeping wave is quite evident for frequencies below about $1 \mathrm{GHz}$. The oscillation due to the interference between the creeping wave and the specular return is essentially gone for $f>2 \mathrm{GHz}$. However, the same sphere embedded in free space would show significant creeping wave interference to nearly $3 \mathrm{GHz}$ (see Fig. 31). The radar cross section of the sphere is behaving as if the frequency were increased by a factor of $\sqrt{\varepsilon_{\text {sand }}} \approx 1.6$.

Fig. 28 - 30 show the radar cross section when the same sphere is embedded in San Antonio clay loam with $5 \%, 10 \%$, and $20 \%$ water by weight. This soil has a higher dielectric constant than dry sand and is a much more lossy medium. As the water content is increased, the loss and the dielectric constant both increase. Evidence of the creeping wave is present only in the soil with $5 \%$ water because of the much higher losses in the other soil models. Clearly, the simple model for large spheres in lossy media is simply

$$
\sigma_{\mu \varepsilon} \cong \pi a^{2}
$$

just as it is for large spheres in free space. Using (127) for the $1 \mathrm{~m}$ diameter sphere embedded in any of the San Antonio clay loam soils results in less than $1 \mathrm{~dB}$ error. Fig. 31 compares the $1 \mathrm{~m}$ diameter sphere in free space with the same sphere embedded in San Antonio clay loam with 20\% water. Obviously, the high-frequency approximation for the radar cross section (127) is valid at much lower frequencies for the sphere embedded in the soil than for the sphere in free space. In fact, a good approximation for the response of the embedded sphere would be the response of the sphere in free space with the frequency scaled by a factor of $\sqrt{\varepsilon_{\text {soil }}} \approx 6$. 


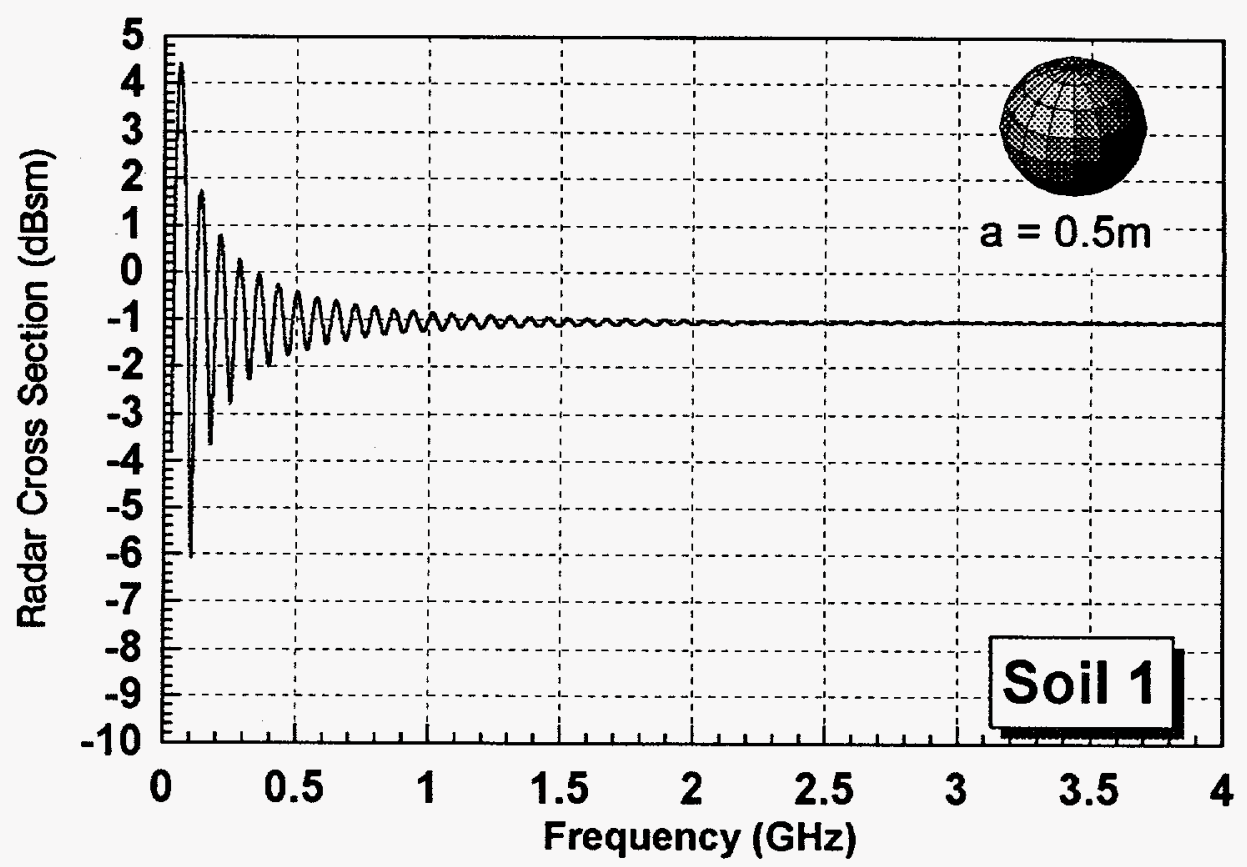

Fig. 27 Monostatic radar cross section of a perfectly conducting sphere of radius 0.5 meters embedded in dry sand.

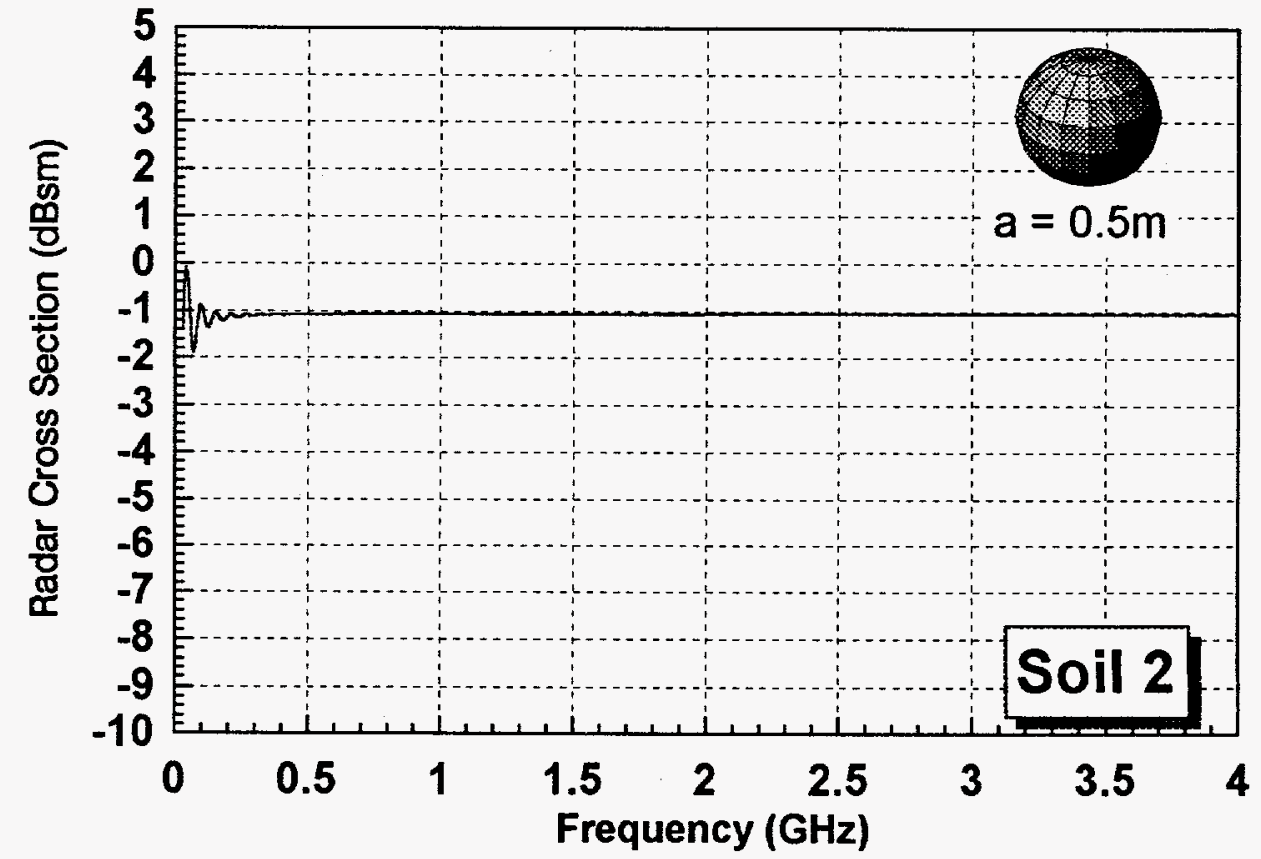

Fig. 28 Monostatic radar cross section of a perfectly conducting sphere of radius 0.5 meters embedded in San Antonio clay loam with 5\% water. 


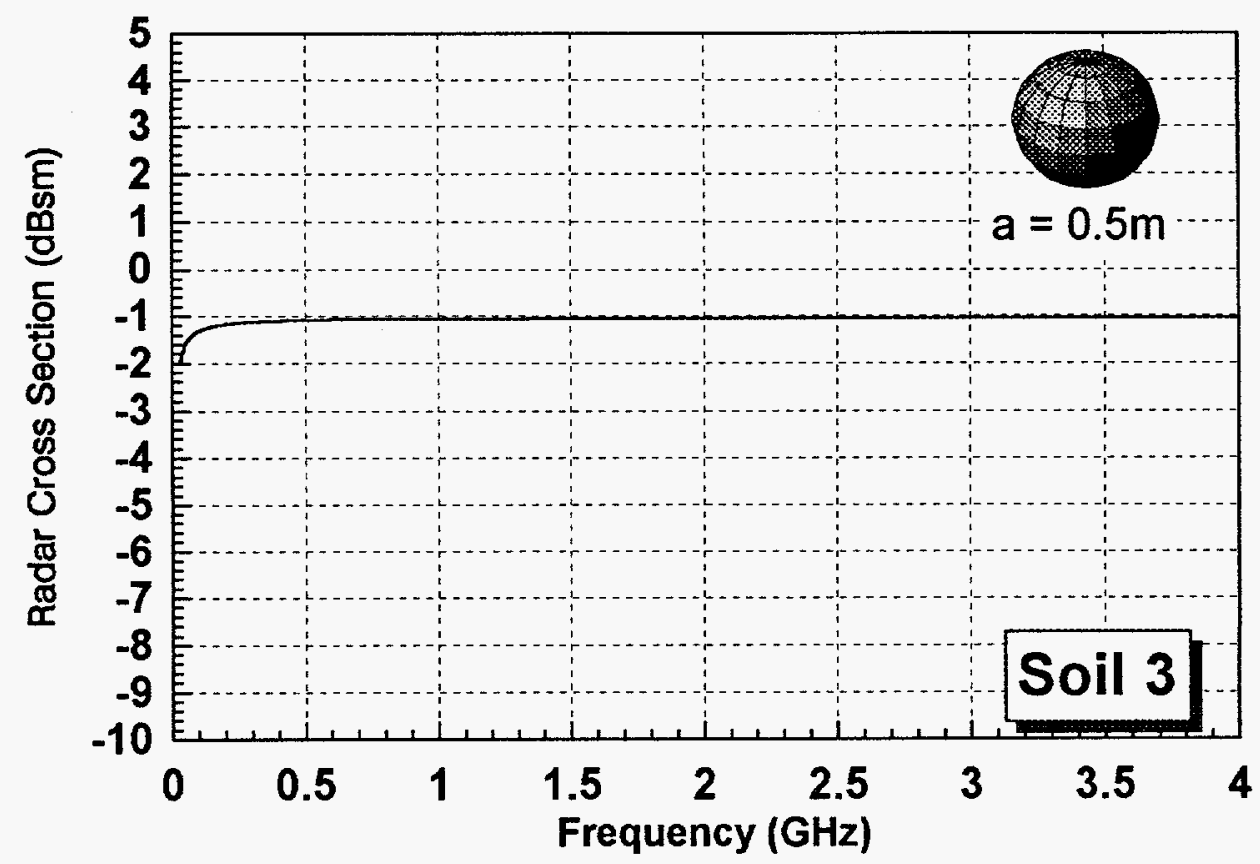

Fig. 29 Monostatic radar cross section of a perfectly conducting sphere of radius 0.5 meters embedded in San Antonio clay loam with 10\% water.

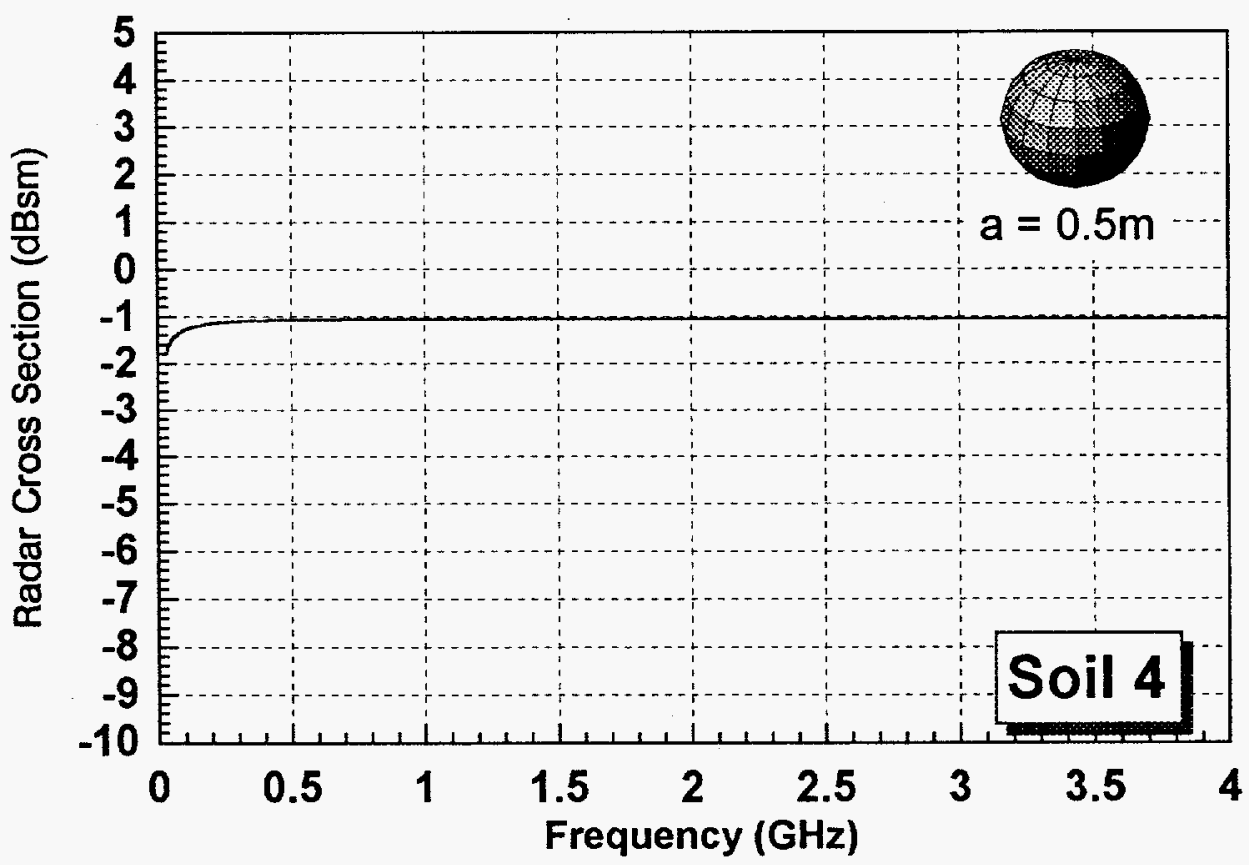

Fig. 30 Monostatic radar cross section of a perfectly conducting sphere of radius 0.5 meters embedded in San Antonio clay loam with $20 \%$ water. 


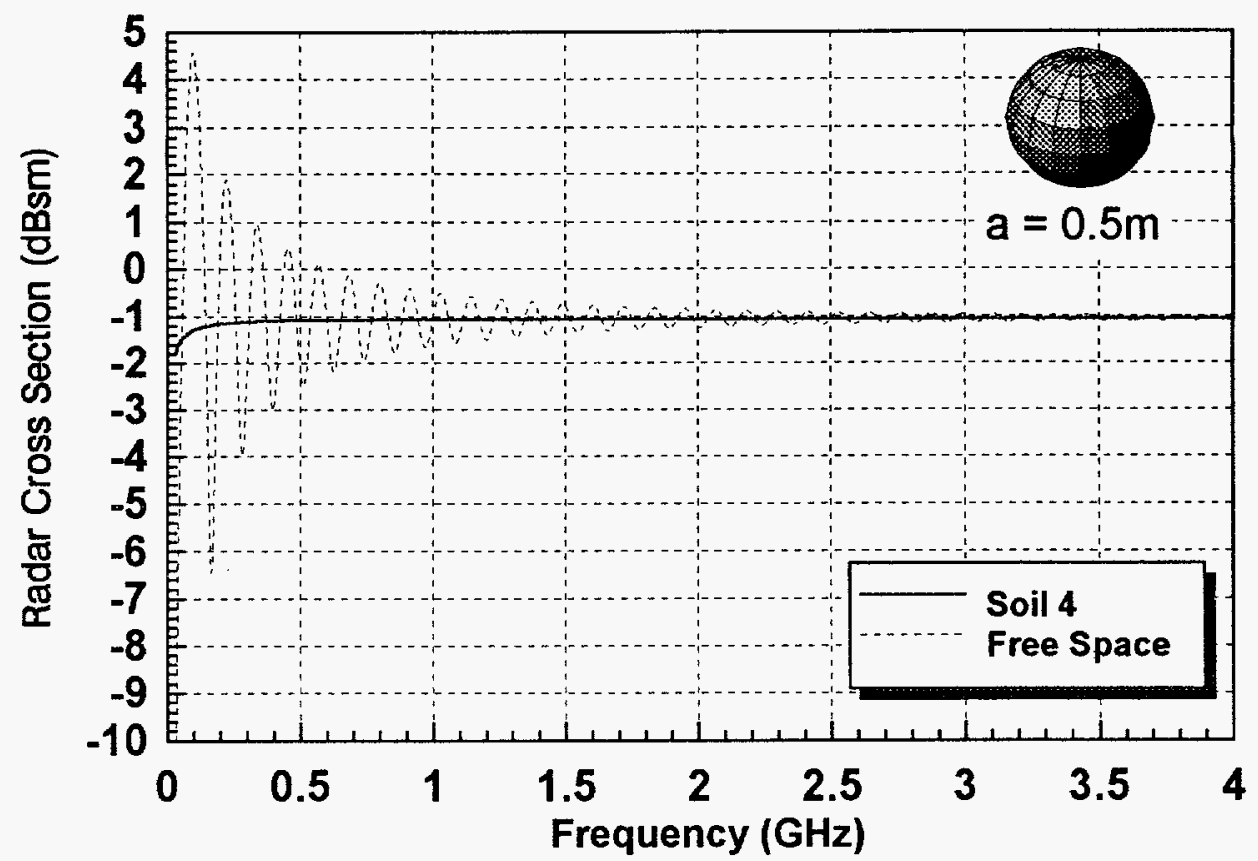

Fig. 31 Comparison of monostatic radar cross sections for a sphere of radius 0.5 meters embedded in San Antonio clay loam with $20 \%$ water vs. free space.

\section{Conclusion}

Electromagnetic imaging of objects buried beneath the surface of the soil is complicated by the dispersion and loss of the soil dielectric. We have demonstrated its effect on loss, resolution, and image contrast. Not only do soil dielectric properties have implications with regard to appropriate frequencies and bandwidths for optimum imaging, the introduction of loss also requires a new definition for the radar cross section. We have introduced a new definition for radar cross section which is valid for objects in lossy media, while being consistent with, and comparable to, the freespace radar cross section definition. This new definition is not only useful, but necessary in order to compare the radar cross section of an object buried in soil with its free-space radar cross section. Finally, we have presented methods of computing this radar cross section for several canonical objects, and have included examples. In summary, the following conclusions and guidelines can be drawn from this study:

- in the absence of surface clutter, lower frequencies are optimum;

- an optimum bandwidth exists;

- loss increases as bandwidth increases;

- very large bandwidths are not useful for imaging objects at large depths;

- in the absence of additional processing, contrast is reduced as depth increases;

- vertical polarization is best for large angles of incidence;

- the appropriate depth for computing losses is the depth to the dominant specular scattering point on the object;

- free-space radar cross sections scaled by the wavelength in the lossy media provide reasonable estimates of the object's buried radar cross section;

- multiple scatterer interactions are less significant for objects buried in lossy media. 
This Page Intentionally Blank 


\section{Appendix A -- Dielectric Soil Models}

A complete description of the model for the soil dielectric constant is contained in [1]; a brief description is included here. The dielectric constant is given by

$$
\varepsilon=\varepsilon_{r} \varepsilon_{0},
$$

where $\varepsilon_{r}$ is the relative dielectric constant (in general complex) and $\varepsilon_{0}$ is the permittivity of free space. The model for $\varepsilon_{r}$ uses the Debye model [2] for the real part of the dielectric constant and the losses due to relaxation in the dielectric, and it also includes a conduction-current loss term. For the case of soil, which is a mixture of various minerals, water, etc., it is reasonable to assume there will be static conductivity and more than one relaxation time constant, so the model used here is

$$
\varepsilon_{r}=\varepsilon_{r \infty o}^{\prime}+\sum_{n} \frac{\Delta \varepsilon_{n}^{\prime}}{1+j \omega \tau_{n}}-j \frac{\sigma_{s}}{\omega \varepsilon_{0}}=\varepsilon_{r}^{\prime}-j \varepsilon_{r}^{\prime \prime},
$$

where $\varepsilon_{r \infty}^{\prime}$ is the relative dielectric constant at very high frequency, $\Delta \varepsilon_{n}^{\prime}$ is the static contribution to the relative dielectric constant from the $n^{\text {th }}$ relaxation term, $\tau_{n}$ is the $n^{\text {th }}$ relaxation time constant, $\omega$ is the angular frequency, and $\sigma_{s}$ is the static conductivity. The real and imaginary parts can be separated

$$
\varepsilon_{r}^{\prime}=\varepsilon_{r \infty}^{\prime}+\sum_{n} \frac{\Delta \varepsilon_{n}^{\prime}}{1+\omega^{2} \tau_{n}^{2}}
$$

and

$$
\varepsilon_{r}^{\prime \prime}=\sum_{n} \frac{\Delta \varepsilon_{n}^{\prime} \omega \tau_{n}}{1+\omega^{2} \tau_{n}^{2}}+\frac{\sigma_{s}}{\omega \varepsilon_{0}} .
$$

Our approach will be to use measured soil dielectric constants obtained from the literature to obtain a fit to $\varepsilon_{r \text { ro }}^{\prime} \Delta \varepsilon_{n}^{\prime}, \tau_{n}$, and $\sigma_{s}$ that will provide a representative soil model for the conditions of interest.

Dry sand has been measured and reported by Von Hippel [7]. Using the parameter-based model above, and assuming that $\varepsilon_{r}^{\prime \prime}$ has only small, smooth variations between the data points reported in [7], the fit shown in Fig. A1-1 can be obtained. The model uses five relaxations and the parameters are shown in Table A1-1.

An example of soil with significant static conductivity is gray San Antonio clay loam as measured by Hipp [10]. Fig. A1-2 shows the real part, $\varepsilon_{r}^{\prime}$, of the relative dielectric constant as a function of frequency for various dry-soil densities and various fractions of water by weight. Fig. A1-3 shows the imaginary part, $\varepsilon_{r}^{\prime \prime}$. Not only is the soil very dispersive, but there is a large variation between the samples. Three cases are chosen to represent this soil: $1.2 \mathrm{~g} / \mathrm{cc}$ dry density with $5 \%$ water by weight, $1.6 \mathrm{~g} / \mathrm{cc}$ dry density with $10 \%$ water by weight, and $1.8 \mathrm{~g} / \mathrm{cc}$ dry density with $20 \%$ water by weight. Using parameter-based modeling, the data was fit with four relaxation terms. The parameters are listed in Table A1-2, and the resulting models are illustrated in Fig. A1-4-6 along with the data points obtained from [10]. 


\begin{tabular}{|c|c|c|c|}
\hline \multicolumn{5}{|c|}{\begin{tabular}{c} 
Table A1-1 \\
Dry Sand \\
Parameters to Fit Data from Von Hippel [7] \\
\hline$\varepsilon_{r o}^{\prime}$
\end{tabular}$\quad \sigma_{s}(\mathrm{~S} / \mathrm{m})$} & $\Delta \varepsilon_{n}^{\prime}$ & $\frac{1}{2 \pi \tau_{n}}(\mathrm{MHz})$ \\
\hline 2.48 & $4.5 \mathrm{E}-07$ & 0.06 & 1 \\
\hline \multicolumn{2}{|c|}{} & 0.058 & 9 \\
\hline & & 0.04 & 80 \\
\hline & & 0.03 & 600 \\
\hline & & 0.02 & 6000 \\
\hline
\end{tabular}

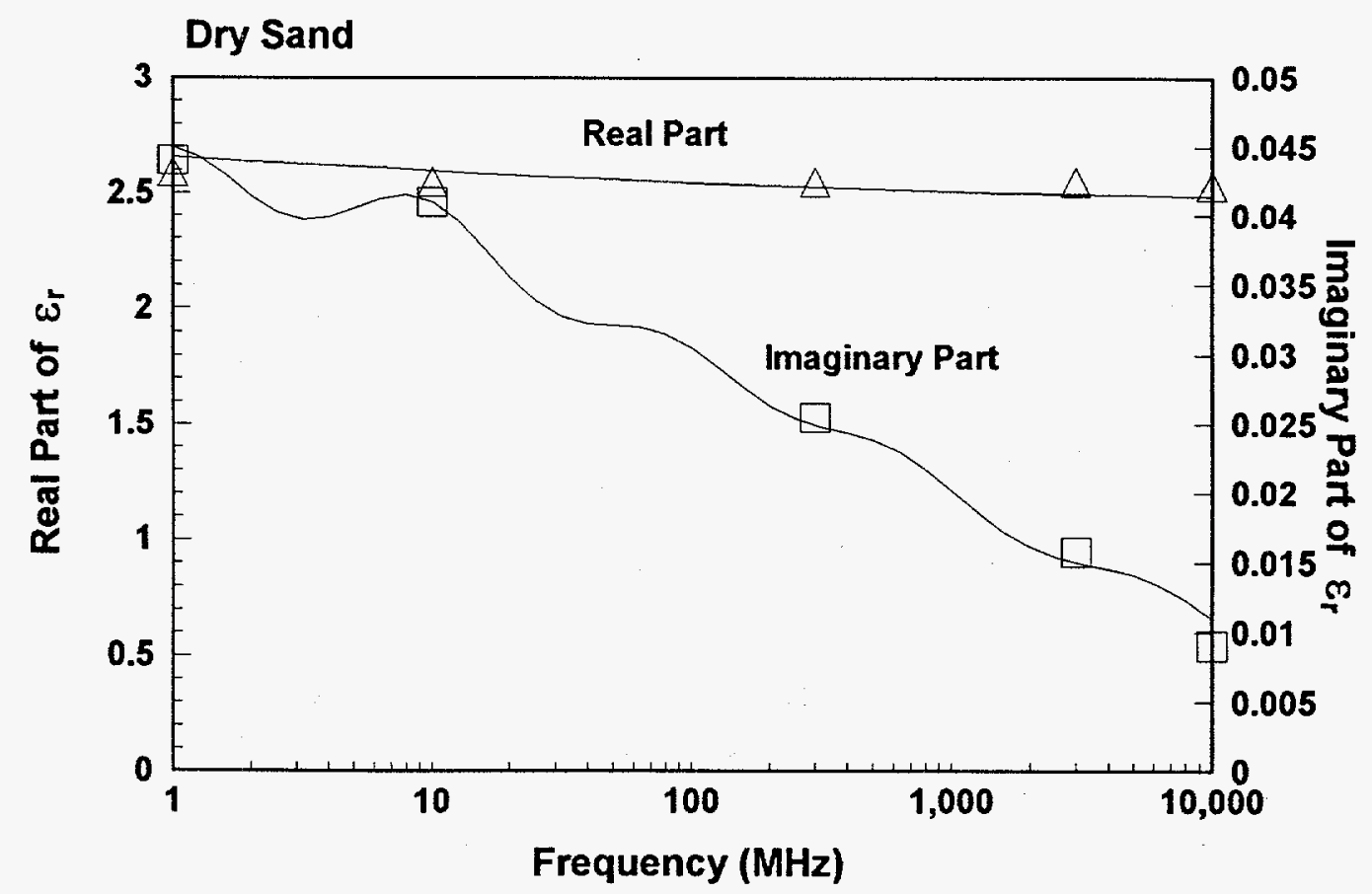

Fig. A1-1 Curve fit for $\varepsilon_{r}$ for dry sand, based on data from Von Hippel [7]. 
J. E. Hipp, 1974, Gray San Antonio Clay Loam

Equivalent Dry Density: $1.2-1.8 \mathrm{~g} /$ cubic $\mathrm{cm}$

Fraction Water by Weight: $5 \%, 10 \%, 20 \%$

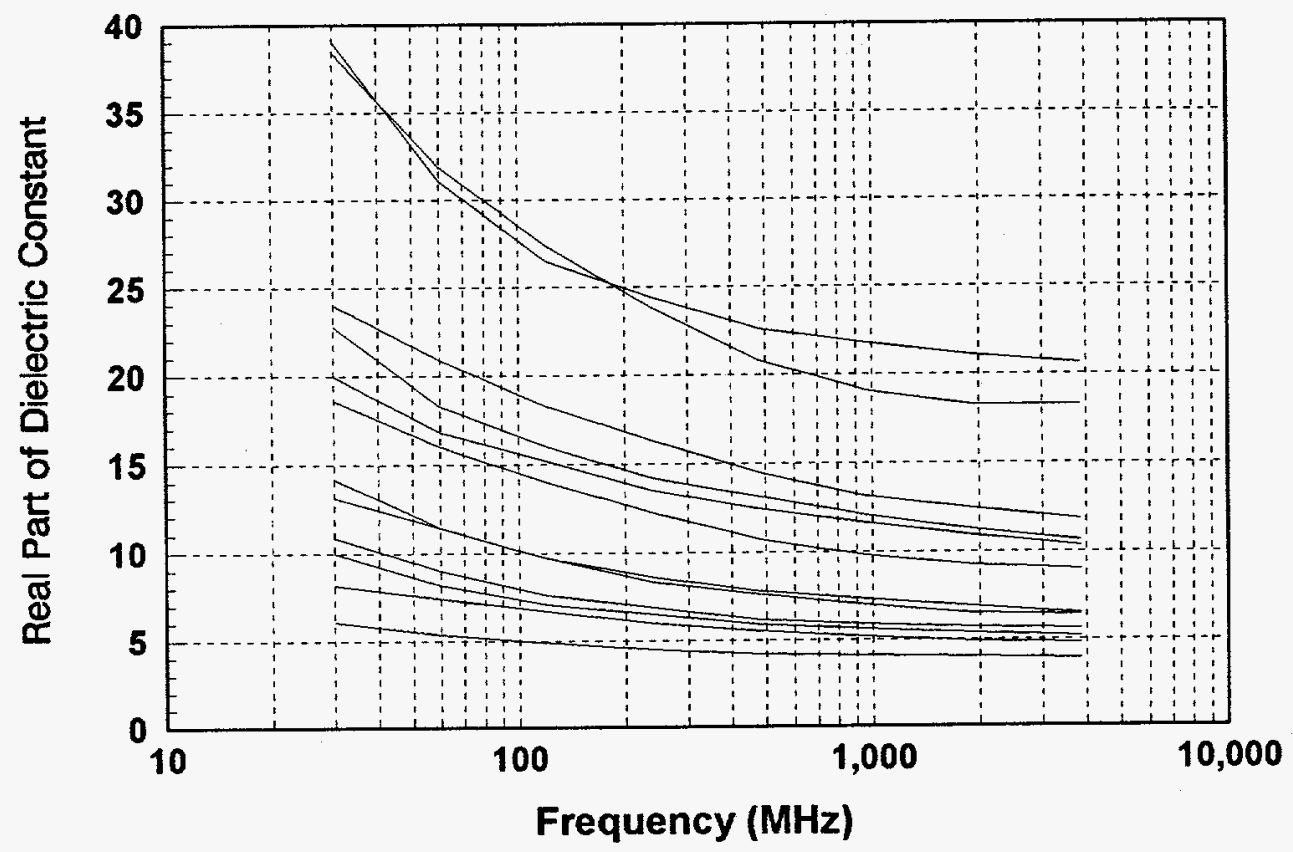

Fig. A1-2 Real part, $\varepsilon_{r}^{\prime}$, of relative dielectric constant for various densities and water content for gray San Antonio clay loam.

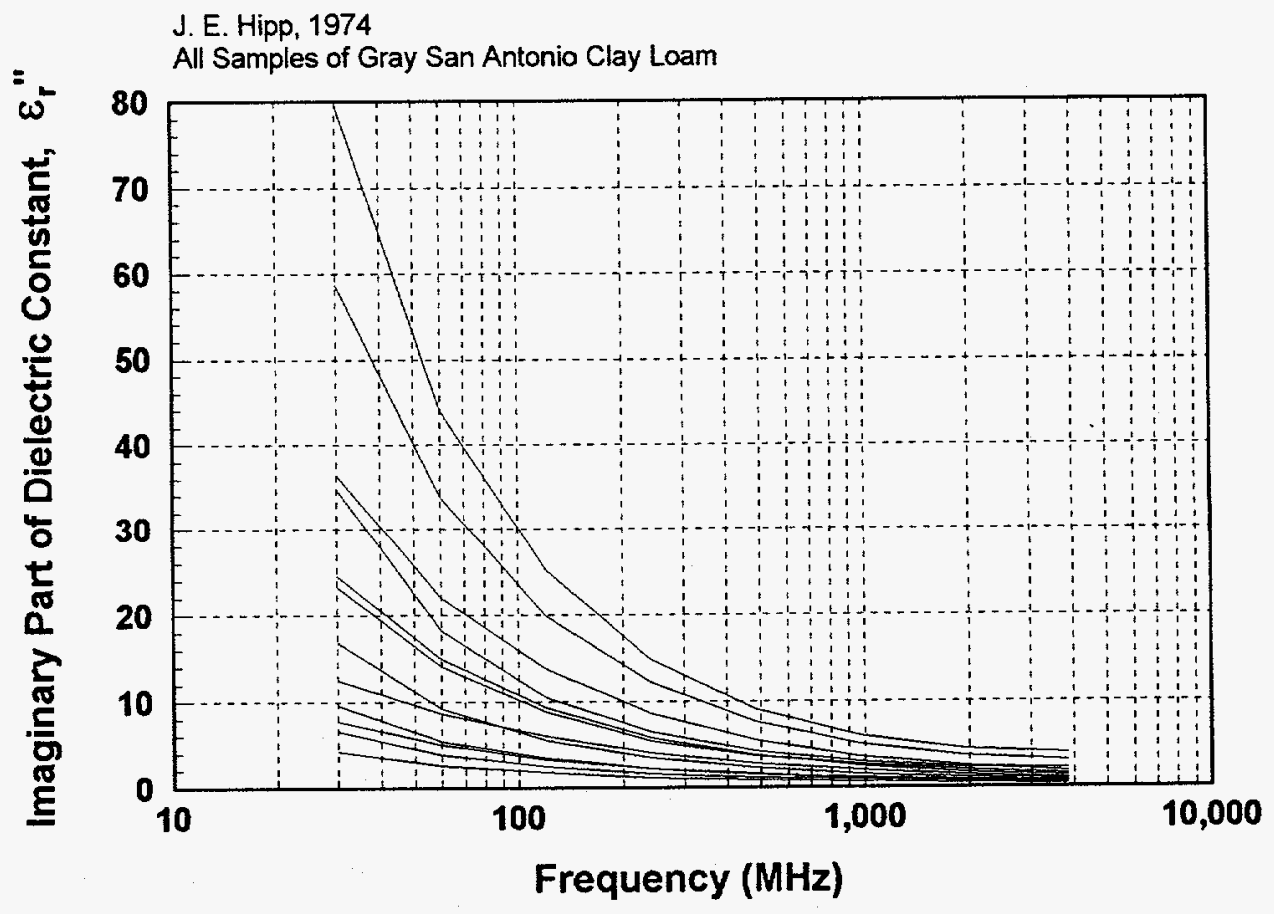

Fig. A1-3 Imaginary part, $\varepsilon_{r}^{\prime \prime}$, of relative dielectric constant for various densities and water content for gray San Antonio clay loam. 


\begin{tabular}{|c|c|c|c|c|c|}
\hline & & $\begin{array}{l}\text { y Sar } \\
\text { leters }\end{array}$ & $\begin{array}{l}\text { ble A1-2 } \\
\text { ntonio Cl } \\
\text { it Data fror }\end{array}$ & $\begin{array}{l}\text { Loam } \\
\operatorname{pp}[10\end{array}$ & \\
\hline $\begin{array}{c}\text { Dry Density } \\
\mathrm{g} / \mathrm{cm}^{3}\end{array}$ & $\begin{array}{l}\text { Water } \\
\text { Content }\end{array}$ & $\varepsilon_{r \infty \infty}^{\prime}$ & $\sigma_{s}(\mathrm{~S} / \mathrm{m})$ & $\Delta \varepsilon_{n}^{\prime}$ & $\frac{1}{2 \pi \tau_{n}}(\mathrm{MHz})$ \\
\hline 1.2 & $5 \%$ & 3.65 & 0.006 & & 35 \\
\hline & & & & 1.20 & 130 \\
\hline & & & & 0.55 & 1200 \\
\hline & & & & 0.15 & 2500 \\
\hline 1.6 & $10 \%$ & 8.3 & 0.036 & 3.5 & 40 \\
\hline & & & & 0.85 & 110 \\
\hline & & & & 2.0 & 1000 \\
\hline & & & & 0.7 & 3000 \\
\hline 1.8 & $20 \%$ & 16.0 & 0.123 & 12.5 & 65 \\
\hline & & & & 3.5 & 110 \\
\hline & & & & 4.8 & 1200 \\
\hline & & & & 2.8 & 6000 \\
\hline
\end{tabular}

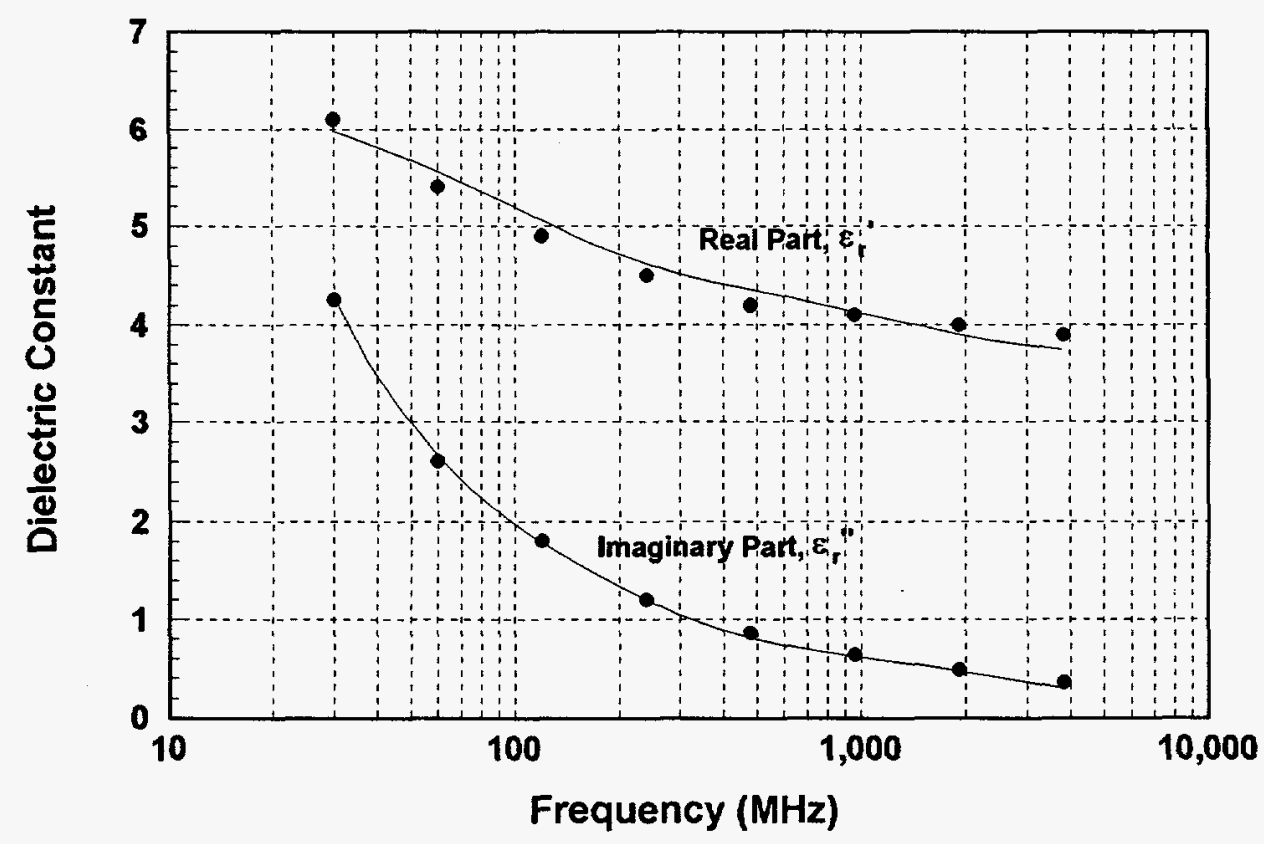

Fig. A1-4 Curve fit (solid line) to data (points) taken from Hipp [10] for gray San Antonio clay loam with dry density of $1.2 \mathrm{~g} / \mathrm{cc}$ with water content $5 \%$ of dry weight. 


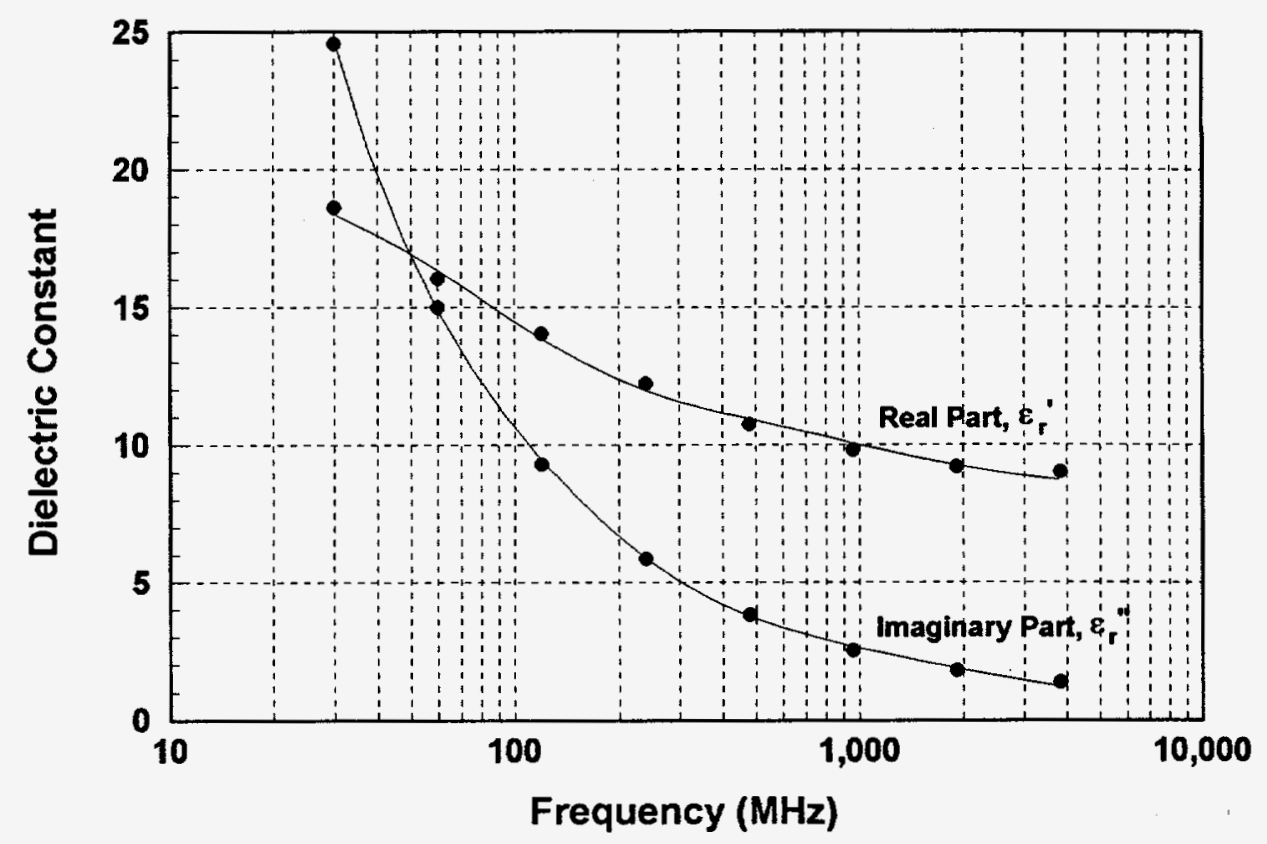

Fig. A1-5 Curve fit (solid line) to data (points) taken from Hipp [10] for gray San Antonio clay loam with dry density of $1.6 \mathrm{~g} / \mathrm{cc}$ with water content $10 \%$ of dry weight.

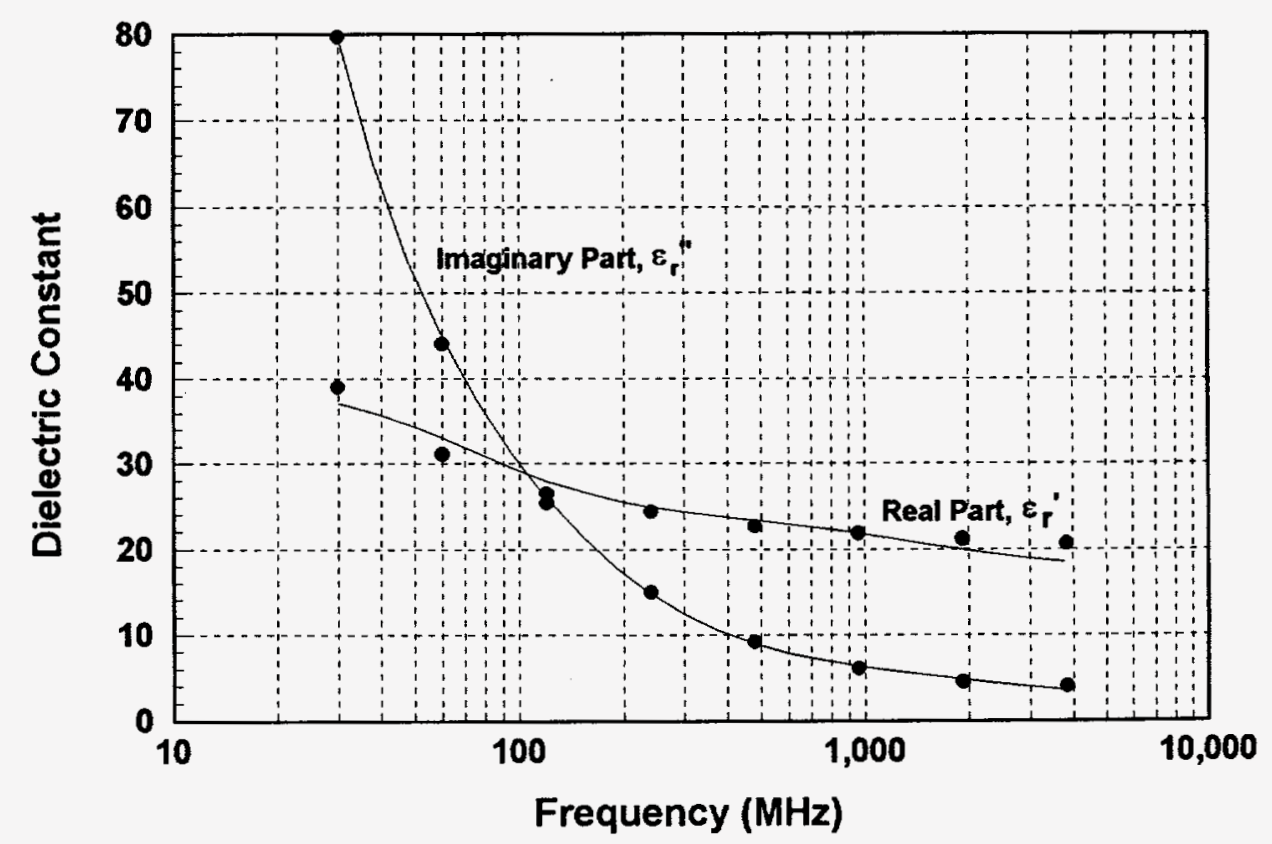

Fig. A1-6 Curve fit (solid line) to data (points) taken from Hipp [10] for gray San Antonio clay loam with dry density of $1.8 \mathrm{~g} / \mathrm{cc}$ with water content $20 \%$ of dry weight. 
This Page Intentionally Blank 


\section{References}

[1] B. C. Brock, W. E. Patitz, Optimum Frequency for Subsurface-Imaging Synthetic Aperture Radar, SAND93-0815, May 1993.

[2] C. A. Balanis, Advanced Engineering Electromagnetics, John Wily \& Sons, New York, 1989.

[3] A. W. Doerry, A Model for Forming Airborne Synthetic Aperture Radar Images of Underground Targets, SAND94-0139, January 1994.

[4] J. D. Jackson, Classical Electrodynamics, John Wiley \& Sons, Inc., New York, 1962.

[5] M. Abramowitz, I. Stegun, Handbook of Mathematical Functions with Formulas, Graphs, and Mathematical Tables, Dover Publications, Inc., New York, 1972 (originally published by the National Bureau of Standards, 1964).

[6] J. Mathews, R. L. Walker, Mathematical Methods of Physics, second edition, The Benjamin/Cummings Publishing Company, Menlo Park, 1970.

[7] A. R. Von Hippel, ed., Dielectric Materials and Applications, The Technology Press of M.I.T. and John Wiley \& Sons, Inc., New York, 1954.

[8] B. O. Peirce, A Short Table of Integrals, Fourth Edition, Ginn and Company, Boston, 1956.

[9] R. F. Harrington, Time-Harmonic Electromagnetic Fields, McGraw-Hill Book Company, New York, 1961.

[10] J. E. Hipp, "Soil Electromagnetic Parameters as Functions of Frequency, Soil Density, and Soil Moisture", Proceedings of the IEEE, vol. 62, no. 1, January 1974, pp. 98-103. 
Distribution:

$\begin{array}{ll}\text { MS-0100 } & \text { Document Processing for DOE/OSTI (7613-2) (10) } \\ \text { MS-0304 } & \text { C. M. Hart (9100) } \\ \text { MS-0355 } & \text { D. H. Cress (9134) } \\ \text { MS-0358 } & \text { B. Boverie (9135) } \\ \text { MS-0471 } & \text { M. W. Callahan (5802) } \\ \text { MS-0509 } & \text { R. D. Andreas (2300) } \\ \text { MS-0509 } & \text { W. D. Williams (2304) } \\ \text { MS-0529 } & \text { B. C. Walker (2345) } \\ \text { MS-0529 } & \text { B. L. Burns (2343) } \\ \text { MS-0529 } & \text { A. W. Doerry (2345) } \\ \text { MS-0529 } & \text { R. B. Hurley (2345) } \\ \text { MS-0531 } & \text { W. H. Schaedla (2343) } \\ \text { MS-0531 } & \text { W. E. Patitz (2343) } \\ \text { MS-0531 } & \text { R. M. Axline (2344) } \\ \text { MS-0531 } & \text { D. L. Bickel (2344) } \\ \text { MS-0531 } & \text { J. T. Cordaro (2344) } \\ \text { MS-0531 } & \text { W. H. Hensley (2344) } \\ \text { MS-0570 } & \text { B. F. Johnson (5900) } \\ \text { MS-0619 } & \text { Technical Publications (7151) } \\ \text { MS-0705 } & \text { T. W. H. Caffey (6114) } \\ \text { MS-0705 } & \text { M. W. Scott (6114) } \\ \text { MS-0899 } & \text { Technical Library (7141) (5) } \\ \text { MS-9018 } & \text { Central Technical Files (8523-2) } \\ \text { MS-0531 } & \text { B. C. Brock (2343) (10) } \\ \text { MS-0531 } & \text { K. W. Sorensen (2343) (2) }\end{array}$

